Supporting information:

\title{
Revealing the Mechanism of Isethionate sulfite-lyase by QM/MM Calculations
}

\author{
Wen-Hao Denga ${ }^{\mathrm{a}}$ You Lu ${ }^{\mathrm{b}^{*}}$, Rong-Zhen Liao ${ }^{\mathrm{a}^{*}}$ \\ ${ }^{a}$ Key Laboratory of Material Chemistry for Energy Conversion and Storage, \\ Ministry of Education, Hubei Key Laboratory of Bioinorganic Chemistry and \\ Materia Medica, Hubei Key Laboratory of Materials Chemistry and Service \\ Failure, School of Chemistry and Chemical Engineering, Huazhong University of \\ Science and Technology, Wuhan 430074, P. R. China \\ bScientific Computing Department, UKRI STFC Daresbury Laboratory, Sci-Tech \\ Daresbury, Warrington WA4 4AD, United Kingdom
}

*Corresponding author: you.lu@stfc.ac.uk; rongzhen@hust.edu.cn 


\section{System preparation using CHARMM version $39 \mathrm{~b} 2$}

\subsection{Preparation of the PDB structure}

The crystal structure of the isethionate sulfite-lyase (IseG, see Figure S1) was selected as the starting structure. ${ }^{1}$ The chain B was selected to construct the model. The glycerol molecules bound to the periphery of this enzyme were deleted. The amide group of Gln775 was flipped based on its local hydrogen-bond network. All Lys and Arg residues were chosen to be protonated at their side chains. All Asp and Glu residues were chosen to be ionized at their side chains. The protonation states of His residues were selected as follows:

HSD (histidine protonated only at ND1): 194, 250

HSE (histidine protonated only at NE2): 405, 531, 562, 573, 773

HSP (histidine protonated at both ND1 and NE2): 76, 88, 152, 164, 289, 371, 414, $510,532,543,552,568,672,683,711,825$



Figure S1. The tetrameric crystal structure of isethionate sulfite-lyase. An enlarged view of chain $B$ is displayed for the critical active-site residues and substrate on the right side. 


\subsection{CHARMM parameter}

Parameters for isethionate and Cys466 are listed below (CHELPG charges were calculated at the B3LYP/def2-SVP level):

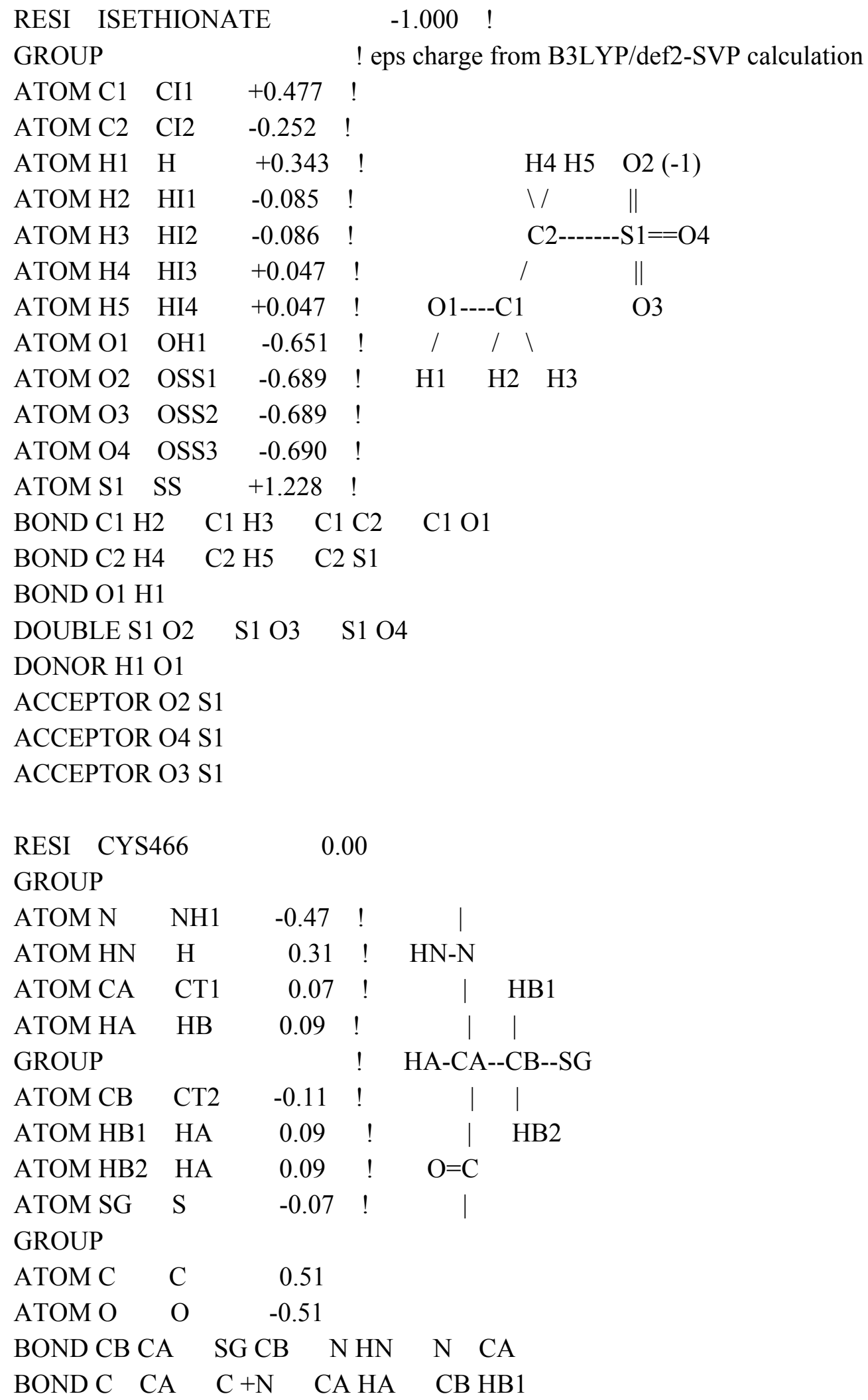


BOND CB HB2

DOUBLE O C

IMPR N -C CA HN C CA + N O

DONOR HN N

ACCEPTOR O C

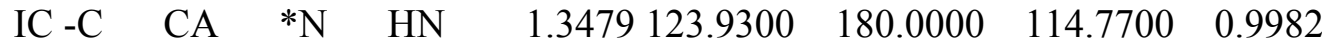

$\begin{array}{lllllllll}\text { IC }-\mathrm{C} & \mathrm{N} & \mathrm{CA} & \mathrm{C} & 1.3479 & 123.9300 & 180.0000 & 105.8900 & 1.5202\end{array}$

$\begin{array}{lllllllll}\mathrm{IC} N & \mathrm{CA} & \mathrm{C} & +\mathrm{N} & 1.4533 & 105.8900 & 180.0000 & 118.3000 & 1.3498\end{array}$

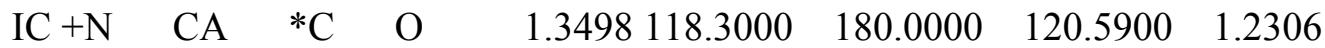

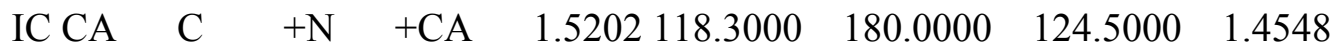

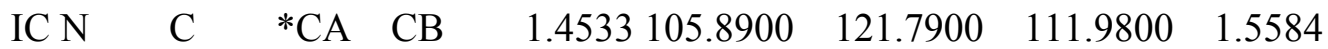

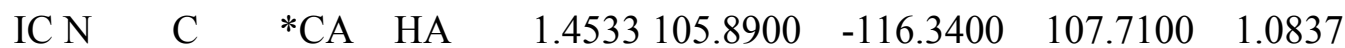

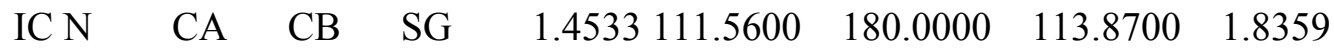

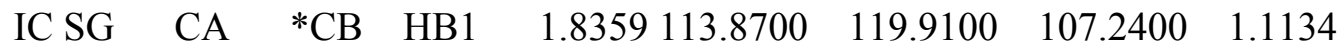






\subsection{Solvation process}

After adding all necessary hydrogen atoms using the HBUILD module in the CHARMM program, ${ }^{2,3}$ the constructed system was first optimized with all non-hydrogen atoms fixed. And some residues, i.e., Arg31, Asn41, Glu341, Lys610, Leu633, Lys758, Tyr794, Arg795, Leu797, and Met828, whose conformations in the crystal structure had not been determined well, were kept free when the constructed system was first optimized. Then, the following solvation and corresponding equilibration processes were done:

(1) System hydration into a water droplet by using a $40 \AA$ sphere of TIP3P water molecules, centered at the sulfur atom of isethionate;

(2) 2000 steps steepest descent (SD) minimization and 10000 steps adopted basis Newton-Raphson (ABNR) minimization;

(3) Heating from $0 \mathrm{~K}$ to $300 \mathrm{~K}$ for 20 ps with a time step of $1 \mathrm{fs}$;

(4) Equilibrating at $300 \mathrm{~K}$ for $30 \mathrm{ps}$ with a time step of $1 \mathrm{fs}$;

(5) Minimization for 5000 steps using the ABNR method.

The final structure was then served as the starting structure for the next solvation process. We repeated the above five steps four times, and the number of water molecules added each time is listed below:

\begin{tabular}{ccccc}
\hline \multicolumn{5}{c}{ Number of water molecules added } \\
\hline Solvation-1 & Solvation-2 & Solvation-3 & Solvation-4 & Solvation-5 \\
4751 & 587 & 270 & 165 & 112 \\
\hline
\end{tabular}




\subsection{Neutralization}

The total charge of the system after hydration is -7 , and seven sodium ions were added close to the protein surface using the CHARMM program. ${ }^{2,3}$ The neutralized system was then subjected to energy minimization and equilibration for $50 \mathrm{ps}$ (20ps heating to $300 \mathrm{~K}$, and $30 \mathrm{ps}$ for equilibrium). Similarly, all non-hydrogen atoms were kept fixed. All sodium ions were on the surface of the enzyme and inside the water droplet. After neutralization, the whole system was subjected to equilibration for 50ps MD simulation (20ps heating to $300 \mathrm{~K}$, and 30 ps for equilibrium) when all non-hydrogen atoms of IseG in this model were fixed. Finally, a geometric optimization for this system was carried out using the ABNR method (5000 steps).

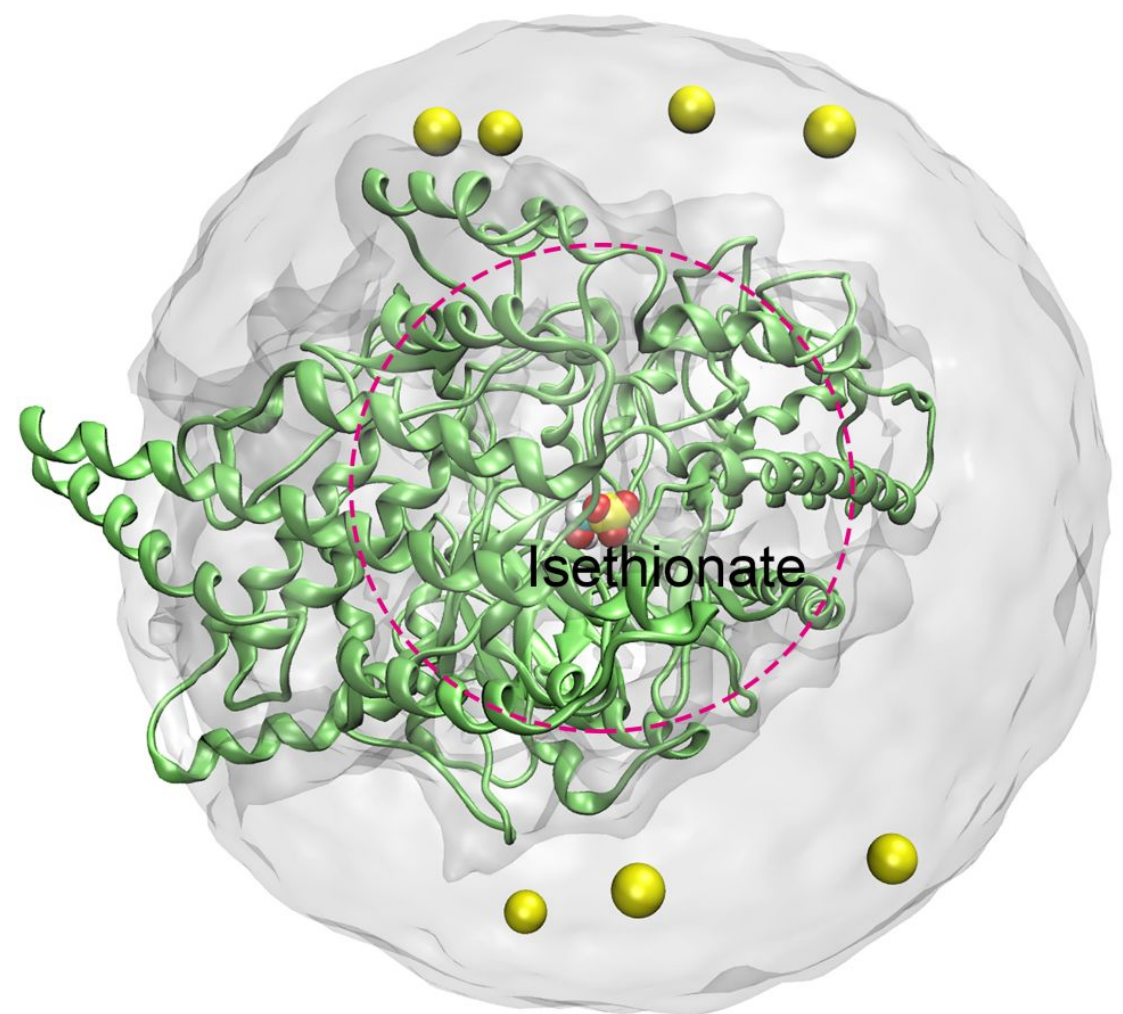

Figure S2. The isethionate sulfite-lyase (IseG) model system built for QM/MM calculations after hydration and neutralization. The inner layer (1168 atoms, within $11 \AA$ of the sulfur atom of isethionate) was allowed to move freely while the outer layer was kept fixed during the geometry optimizations. The seven sodium ions added were shown in yellow ball. 


\subsection{Active region}

The following residues (within $11 \AA ̊$ of the sulfur atom of isethionate, 1168 atoms) were allowed to move during the QM/MM geometry optimizations:

Residues:

Asn181; Glu182; Thr183; Ser184; Ser185; Phe186; Arg187; Ser188; Ser189; Ile190; Gln191; Trp192; Val193; Lys307; Thr308; Gly309; Thr310; Ile311; Val312; Ser313; Asp355; Leu356; Tyr357; Phe365; Asn366; Trp372; Glu373; Ala374; Lys437; Tyr448; Val463; Ser464; Gly465; CYR466; Thr467; Glu468; Ala469; Arg470; Met471; Pro472; Asn473; Arg474; Thr475; Tyr476; Thr477; Ser478; Ala479; Gly480; Gly481; Ala482; Thr483; Ile484; Gln542; HSP543; Ile544; Ile 545; Asn546; Asn547; Leu548; Arg549; Leu583; Gly584; Tyr585; Phe586; Glu587; Tyr588; Met589; Met671; HSP 672; Asn673; Asp674; Val675; Arg676; Tyr677; Val 678; Pro 679; Phe680; Thr681; Ser682; Ala737; Met740; Trp772; Ala802; Gly803.

Water molecules:

Crystal water 7; Crystal water 11; Crystal water 17; Crystal water 42; Crystal water 43; Crystal water 52; Crystal water 61; Crystal water 76; Crystal water 91; Crystal water 100; Crystal water 109; Crystal water 112; Crystal water 123; Crystal water 124; Crystal water 128; Crystal water 153; Crystal water 172; Crystal water 177; Crystal water 178; Solvent water 3. 


\section{2. $Q M / M M$ calculations on the degradation of isethionate}

\subsection{Calculated spin densities on key atoms}

Table S1. Calculated spin densities on key atoms in the QM region at the B3LYP-D3/def2-TZVPP:Charmm level.

\begin{tabular}{ccccccccc}
\hline atom & S1 & $\mathbf{C 1}$ & $\mathbf{C 2}$ & $\mathbf{0 1}$ & $\mathbf{S 2}$ & $\mathbf{0 3}$ & $\mathbf{0 4}$ & $\mathbf{0 5}$ \\
\hline React & 0.82 & 0 & 0.06 & 0.02 & 0 & 0 & 0 & 0 \\
TS1 & 0.33 & 0 & 0.46 & 0.14 & 0.04 & 0 & 0.01 & 0.02 \\
Int1 & 0.07 & -0.03 & 0.62 & 0.21 & 0.07 & 0 & 0.02 & 0.02 \\
TS2 & 0.02 & -0.05 & 0.40 & 0.14 & 0.24 & 0.04 & 0.10 & 0.10 \\
Int2b & 0.01 & 0.04 & 0.14 & 0.06 & 0.30 & 0.11 & 0.15 & 0.16 \\
TS3b & 0.01 & 0.69 & -0.09 & -0.01 & 0.18 & 0.08 & 0.07 & 0.04 \\
Int3b & 0.01 & 0.86 & -0.03 & 0 & 0.08 & 0.03 & 0.02 & 0.01 \\
TS4b & 0.02 & 0.86 & -0.03 & 0 & 0.08 & 0.03 & 0.01 & 0.01 \\
Int4b & 0.04 & 0.83 & -0.03 & 0 & 0.08 & 0.02 & 0.02 & 0.01 \\
TS5b & 0.30 & 0.64 & -0.02 & 0 & 0.05 & 0.02 & 0.02 & 0.01 \\
Int5b & 0.88 & 0.02 & 0 & 0 & 0 & 0 & 0 & 0 \\
\hline
\end{tabular}




\subsection{Calculate energies for the migration mechanism}

Table S2. $Q M, M M$ and $Q M / M M$ relative energies (in $\mathrm{kcal} / \mathrm{mol}$ ) calculated using three different density functionals with the def2-TZVPP basis set. Zero-point energies (in $\mathrm{kcal} / \mathrm{mol}$ ) obtained by numerical frequency calculations at B3LYP-D3/def2-SVP:Charmm level are displayed in parentheses.

\begin{tabular}{cccccccccc}
\hline & \multicolumn{3}{c}{ B3LYP-D3 } & \multicolumn{3}{c}{ M06-D3 } & \multicolumn{3}{c}{ TPSSh-D3 } \\
\hline & QM & MM & QM/MM & QM & MM & QM/MM & QM & MM & QM/MM \\
\hline React & 0.0 & 0.0 & $0.0(0.0)$ & 0.0 & 0.0 & 0.0 & 0.0 & 0.0 & 0.0 \\
TS1 & 10.5 & -1.2 & $9.3(-4.0)$ & 13.3 & -1.2 & 12.1 & 8.5 & -1.2 & 7.3 \\
Int1 & 5.8 & 0.4 & $6.2(-2.2)$ & 6.9 & 0.5 & 7.4 & 4.6 & 0.5 & 5.1 \\
TS2b & 9.8 & 2.2 & $12.0(-3.3)$ & 13.6 & 2.2 & 15.8 & 8.8 & 2.2 & 11.0 \\
Int2b & 8.2 & 3.0 & $11.2(-3.0)$ & 13.5 & 3.0 & 16.5 & 8.0 & 3.0 & 11.0 \\
TS3b & 16.6 & 1.0 & $17.6(-2.7)$ & 17.4 & 1.0 & 18.4 & 15.5 & 1.0 & 16.5 \\
Int3b & 13.4 & 0.6 & $14.0(-2.9)$ & 10.8 & 0.6 & 11.4 & 12.5 & 0.6 & 13.1 \\
TS4b & 15.4 & 1.4 & $16.8(-3.1)$ & 13.0 & 1.4 & 14.4 & 14.8 & 1.4 & 16.2 \\
Int4b & 12.6 & 1.7 & $14.3(-3.0)$ & 10.2 & 1.6 & 11.8 & 11.8 & 1.6 & 13.4 \\
TS5b & 14.7 & 2.9 & $17.6(-4.5)$ & 14.5 & 2.9 & 17.4 & 13.0 & 2.9 & 15.9 \\
Int5b & -2.8 & 2.0 & $-0.8(-0.4)$ & -4.4 & 2.1 & -2.3 & -2.2 & 2.1 & -0.1 \\
\hline
\end{tabular}


Table S3. Relative Gibbs energies (in $\mathrm{kcal} / \mathrm{mol}$ ) calculated using three different density functionals with the def2-TZVPP basis sets for the final elimination step outside the enzyme: (A) One water molecule participates in the elimination of 1-hydroxyethane-1-sulfonate (HES) in an aqueous solution. (B) Two water molecules participate in the elimination of 1-hydroxyethane-1-sulfonate (HES) in an aqueous solution.

\begin{tabular}{c|cccc}
\hline \multicolumn{1}{c}{ Species } & B3LYP-D3 & M06-D3 & TPSSh-D3 \\
\hline \multirow{4}{*}{ A } & HES+W1 & 0.0 & 0.0 & 0.0 \\
& TS5' $_{\text {w1 }}$ & 18.9 & 23.2 & 17.5 \\
& Prod"'+W1 & 0.9 & 5.4 & 4.9 \\
\hline \multirow{3}{*}{ B } & HES+W2 & 0.0 & 0.0 & 0.0 \\
& TS5' ${ }^{\text {w2 }}$ & 18.8 & 24.4 & 17.3 \\
& Prod"'+W2 & 0.9 & 5.4 & 4.9 \\
\hline
\end{tabular}


Table S4. $Q M, M M$ and $Q M / M M$ relative energies (in $\mathrm{kcal} / \mathrm{mol}$ ) calculated at the B3LYP-D3/def2-TZVPP:Charmm level: (A) The active region of 1168 atoms in total was selected, containing all atoms within $11 \AA$ from the sulfur atom of isethionate. (B) The active region of 2306 atoms in total was selected, containing all atoms within 15 A from the sulfur atom of isethionate.

\begin{tabular}{c|cccc}
\hline \multicolumn{1}{c}{ Species } & QM & MM & QM/MM \\
\hline \multirow{4}{*}{ A } & React & 0 & 0 & 0 \\
& TS3b & 16.6 & 1.0 & 17.6 \\
& TS5b & 14.7 & 2.9 & 17.6 \\
\hline \multirow{3}{*}{ B } & React & 0.0 & 0.0 & 0.0 \\
& TS3b & 15.9 & 1.3 & 17.2 \\
& TS5b & 14.5 & 2.1 & 16.6 \\
\hline
\end{tabular}




\subsection{All optimized structures of $\mathrm{QM}$ region}

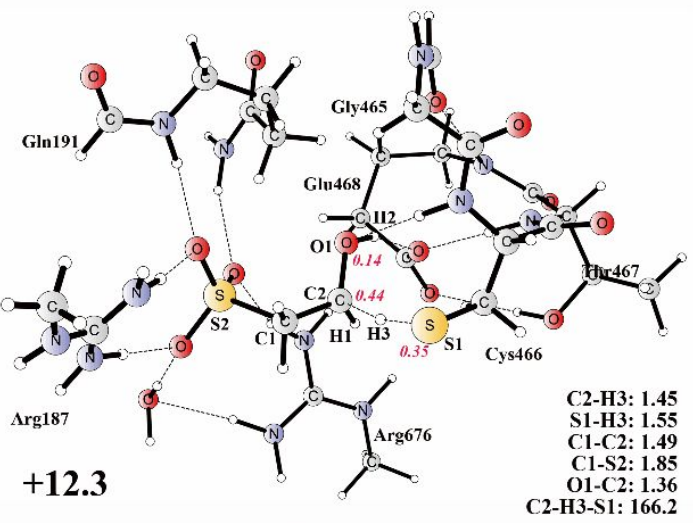

TS1



Int1'

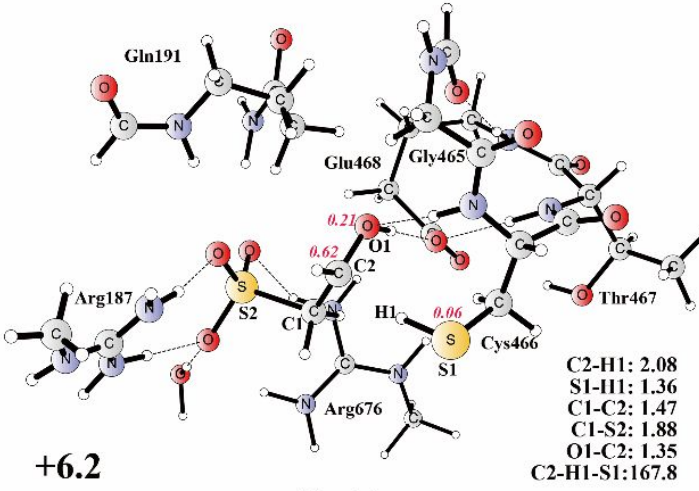

Int1

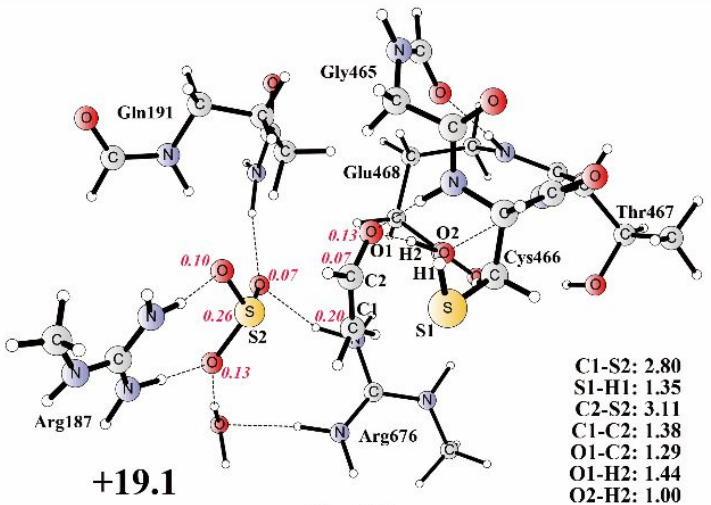

Int2a

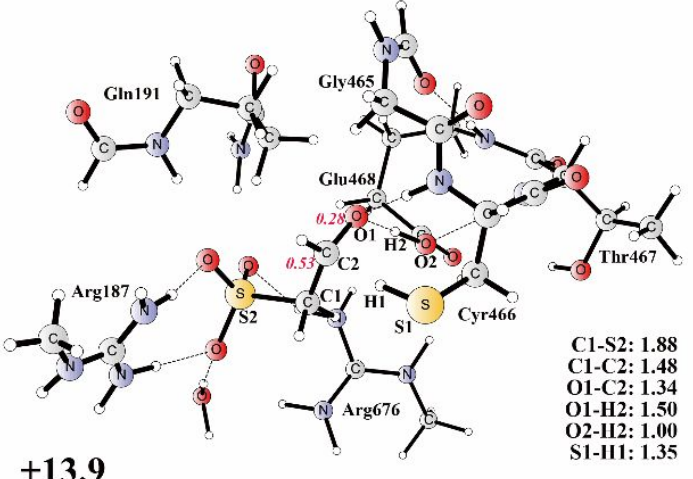

$+13.9$

Int2a'

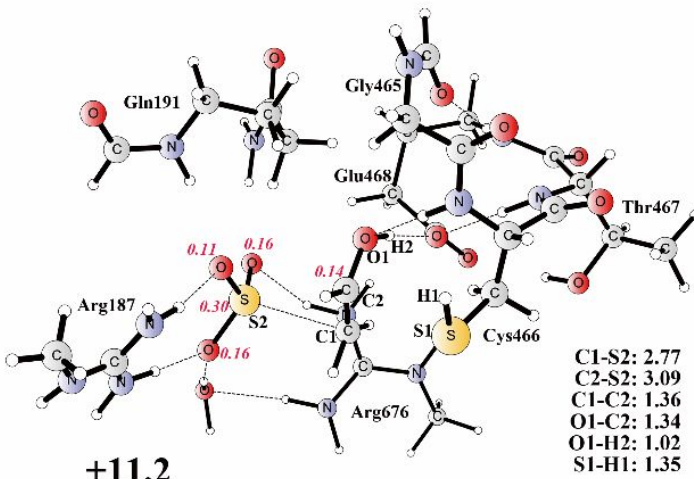

Int2b

Figure S3. QM/MM optimized structures of several intermediates and transition state. Selected parameters are shown in the picture. The spin densities on key atoms are shown in red. All distances are given in $A$, and angles are in degrees. Energies evaluated at the B3LYP-D3/def2-TZVPP:Charmm level are given in $\mathrm{kcal} / \mathrm{mol}$ relative to React. 




Figure S4. Detailed optimized QM/MM geometries (core region) of the reactant, intermediates, and transition states for the migration mechanism. Mulliken spin densities are shown in red italic. The 3D spin densities (green) of key atoms are shown at an isovalue of 0.01 a.u. 

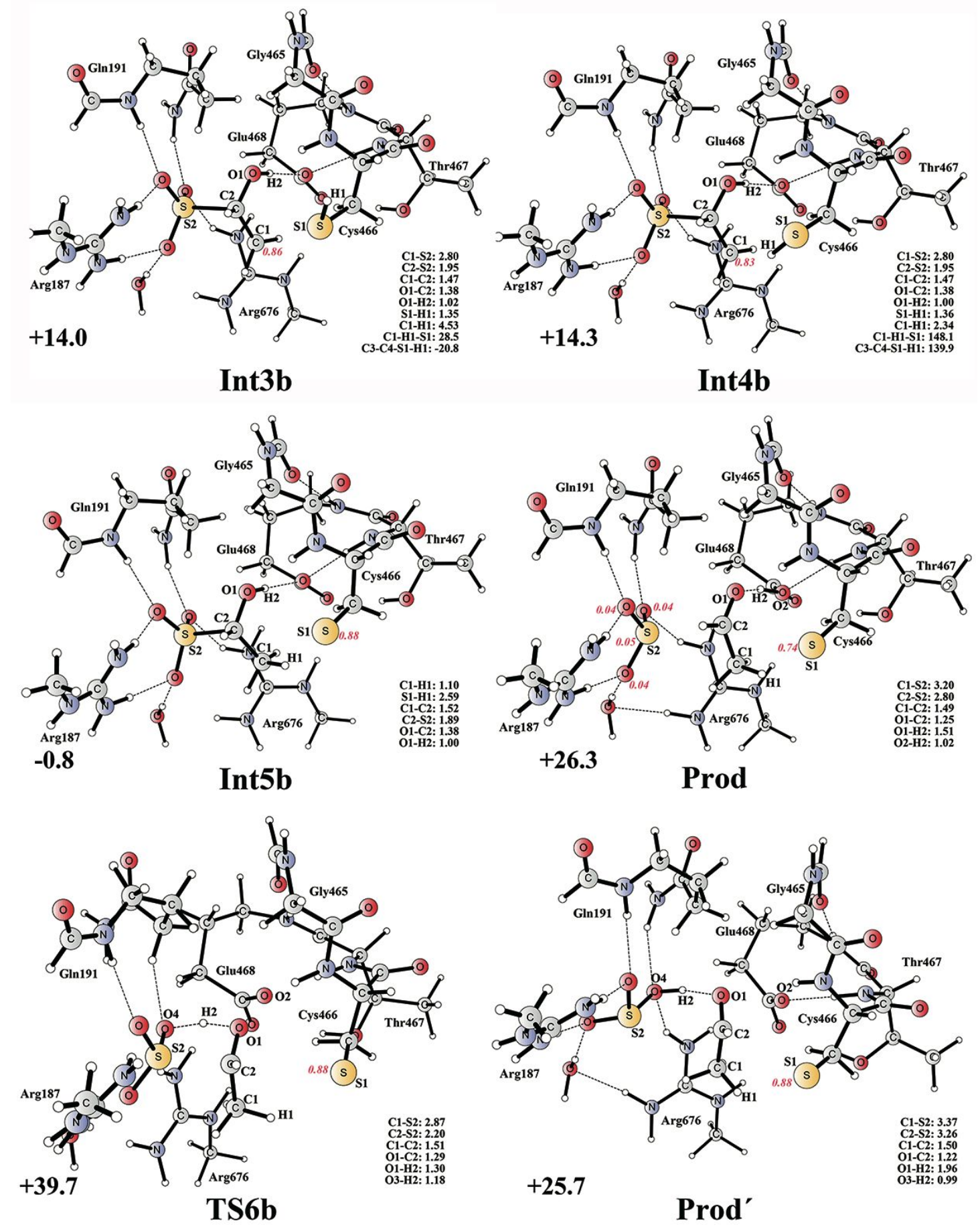

Figure S5. QM/MM optimized structures of several discussed intermediates and transition state. Selected parameters are shown in the picture. The spin densities on key atoms are in red. All distances are given in A. Energies evaluated at the B3LYP-D3/def2-TZVPP:Charmm level are given in $\mathrm{kcal} / \mathrm{mol}$ relative to React. 


\subsection{Potential energy profiles}

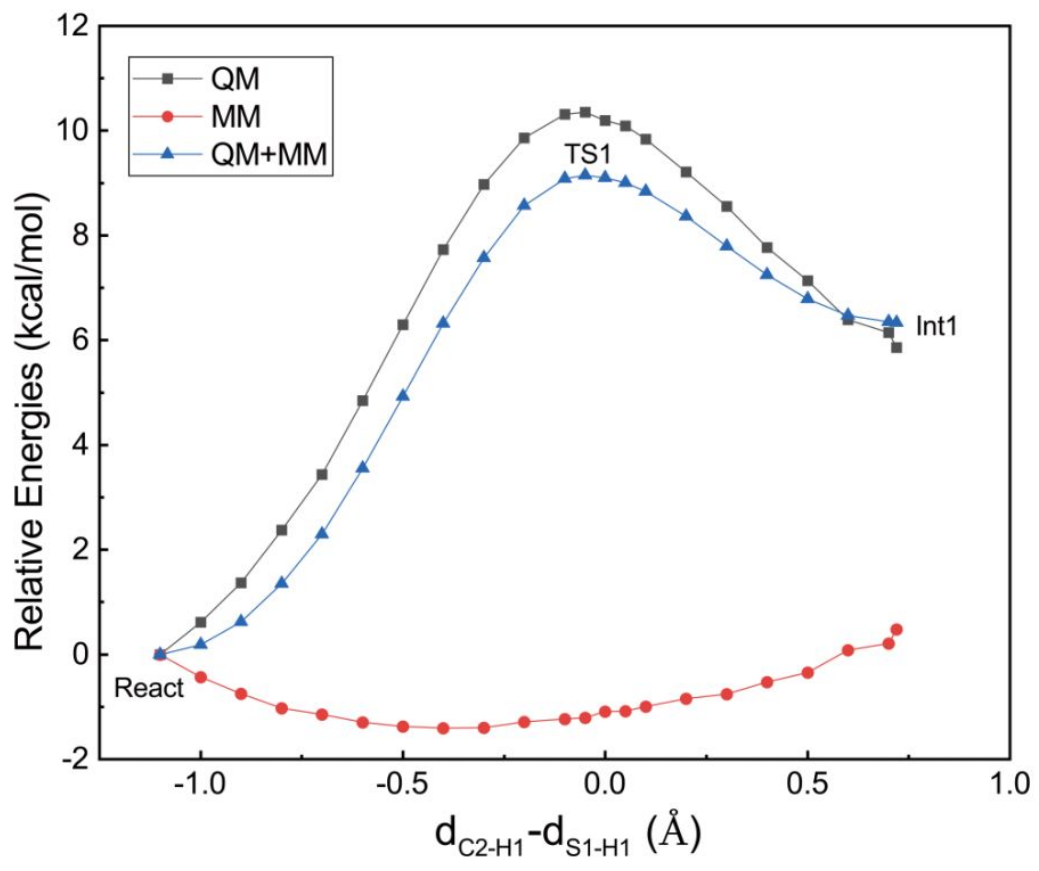

Figure S6. Potential energy profile for the first hydrogen atom transfer step calculated at the B3LYP-D3/def2-SVP:Charmm level using $d_{C 2-H 1}-d_{S 1-H 1}$ as the reaction coordinate.

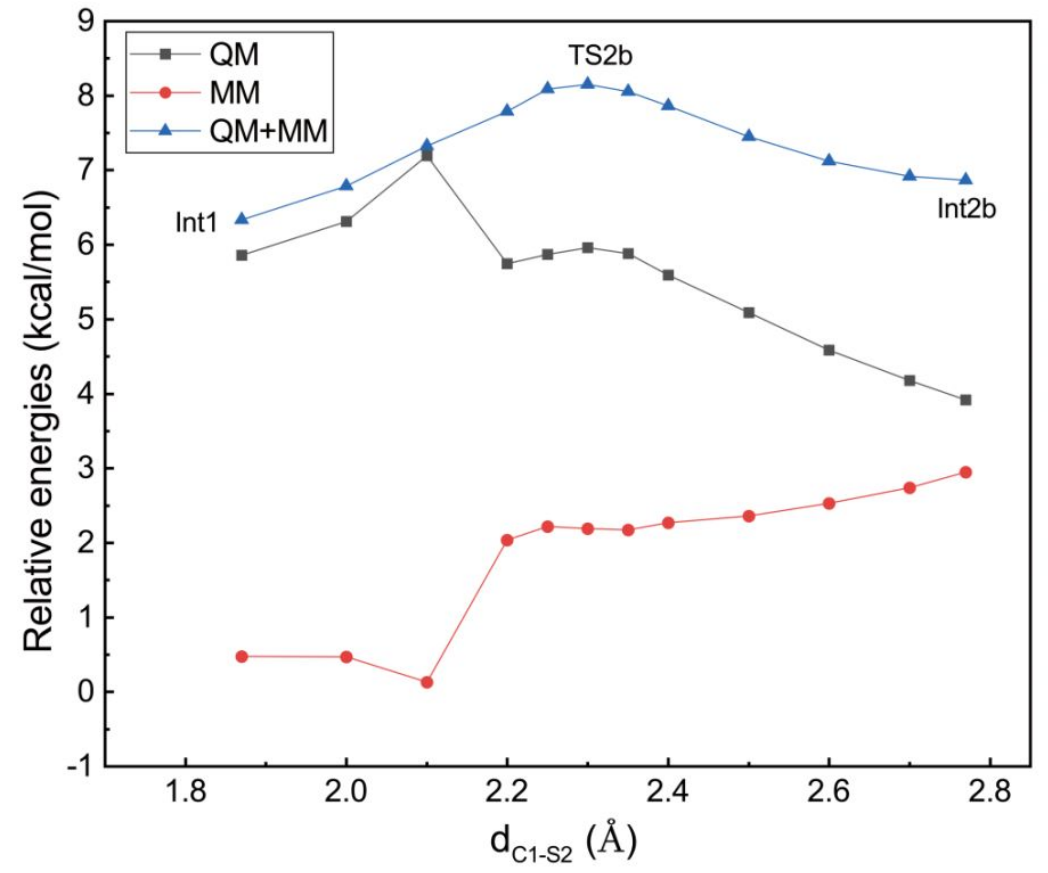

Figure S7. Potential energy profile for the fragmentation step calculated at the B3LYP-D3/def2-SVP:Charmm level using $d_{C 1-S 2}$ as the reaction coordinate. 




Figure S8. Potential energy profile for the recombination step calculated at the B3LYP-D3/def2-SVP:Charmm level using $d_{C 2-S 2}$ as the reaction coordinate.

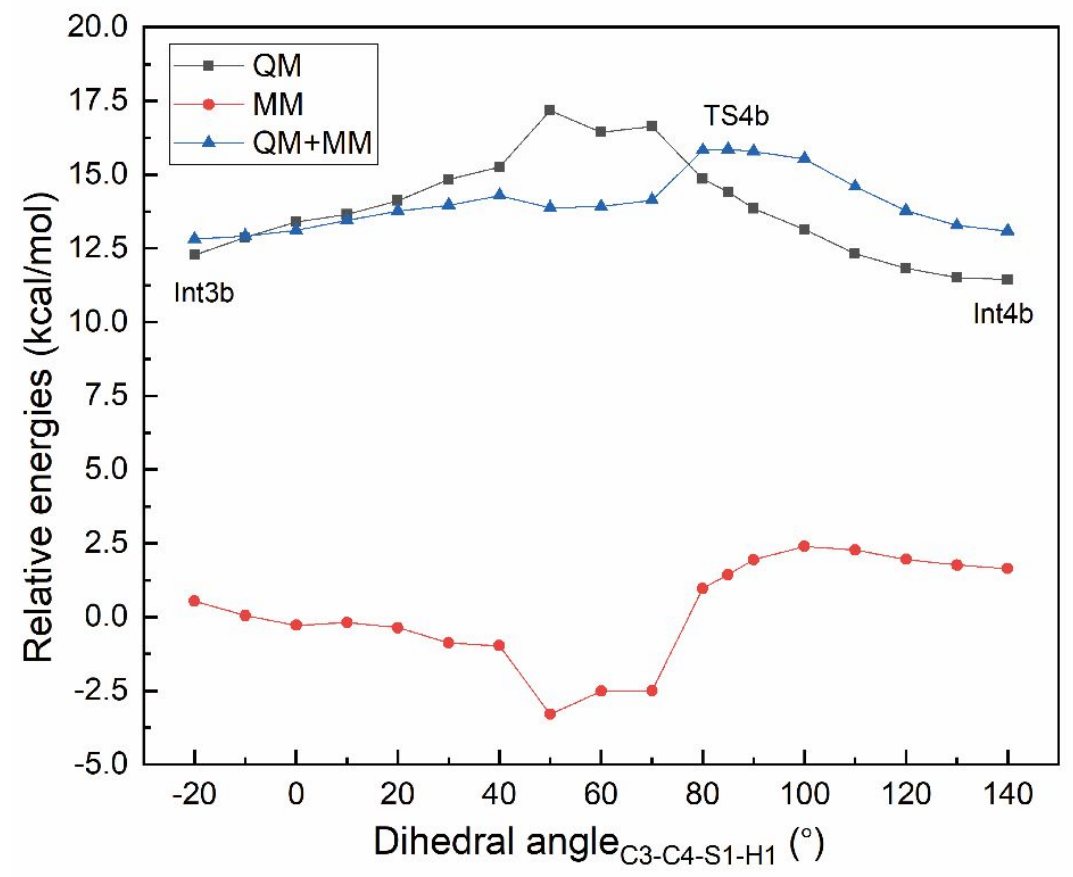

Figure S9. Potential energy profile for the rotation of Cys466's thiol group calculated at the B3LYP-D3/def2-SVP:Charmm level using the C3-C4-S1-H1 dihedral angle as the reaction coordinate. 


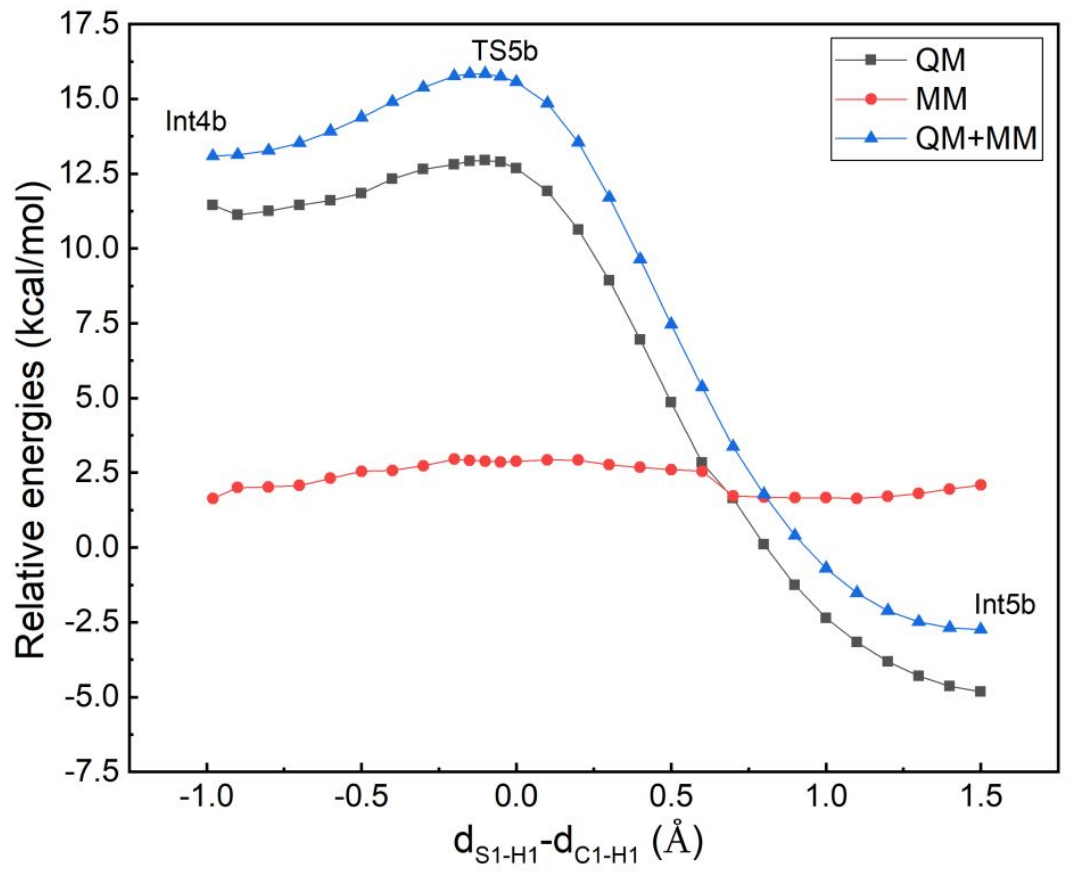

Figure S10. Potential energy profile for the second hydrogen atom transfer step calculated at the B3LYP-D3/def2-SVP:Charmm level using $d_{S 1-H_{1}-d_{C 1-H 1}}$ as the reaction coordinate.

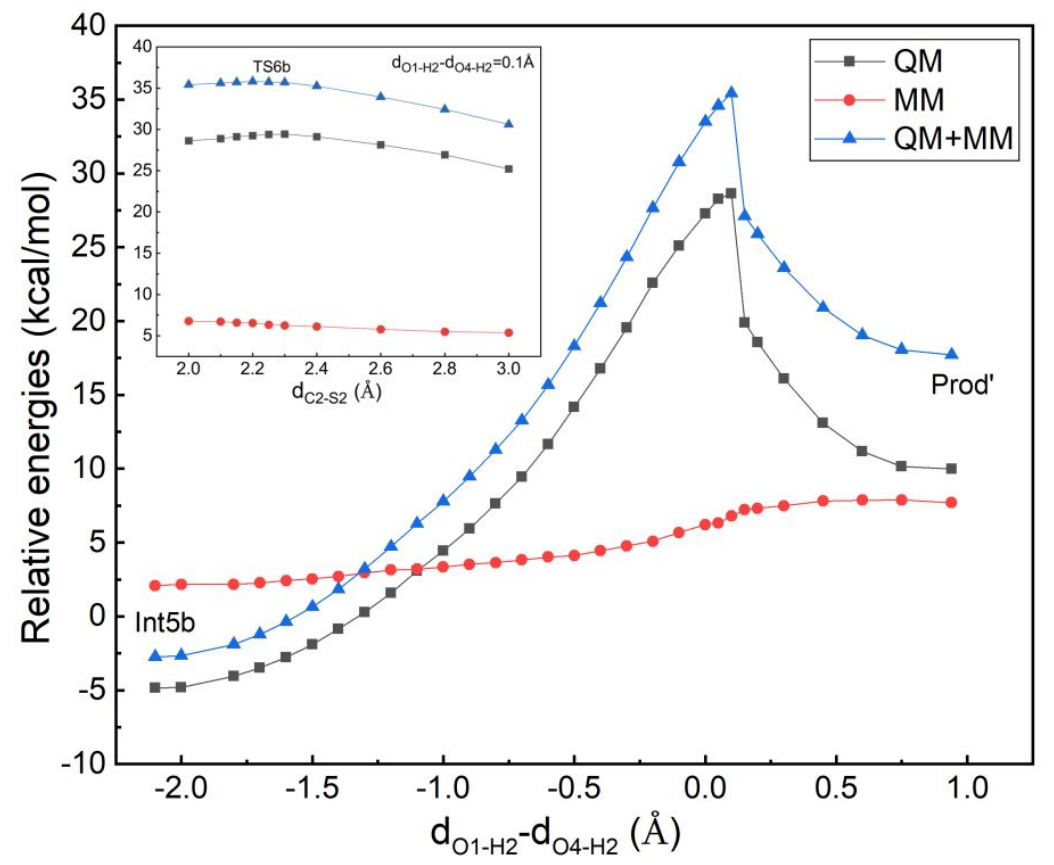

Figure S11. Potential energy profile for the five-cycle elimination step in binding pocket calculated at the B3LYP-D3/def2-SVP:Charmm level using $d_{01-\mathrm{H}^{-}} \mathrm{d}_{04-\mathrm{H} 2}$ as the reaction coordinate. The results reveal that it is a concerted but asynchronous process. 




Figure S12. One-electron oxidation potentials (vS SHE) for sufite ( $A, B, C$ and $D)$ and vinyl alcohol ( $E, F$ and $G$ ). Different residues and water molecules on the influence of the oxidation potentials calculated in water solution $(\varepsilon=81)$ are considered. The calculated oxidation potentials values (VS SHE) in a low dielectric environment $(\varepsilon=4)$ were shown in parentheses. All calculations were carried out at the B3LYP-D3/def2-TZVPP level. 
A

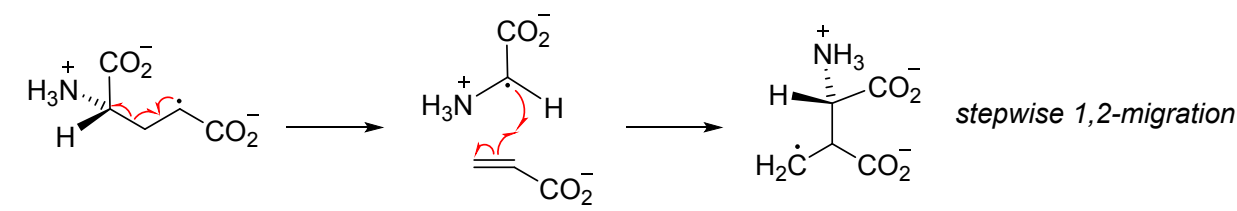

B<smiles>[CH]C([NH-])O</smiles>

concerted 1,2-migration

C

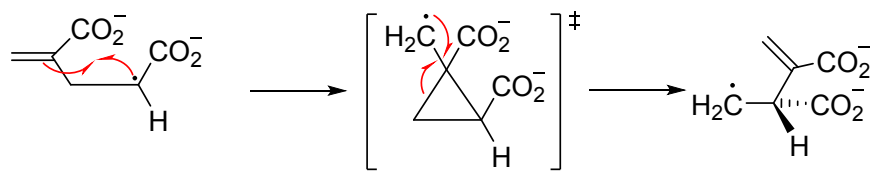

concerted 1,2-migration

Figure S13. Previously proposed mechanisms for free-radical-based 1,2-migrations in three different $B_{12}$-dependent enzymes. (A) Fragmentation-recombination mechanism of glutamate mutase (GM);4 (B) 1,2-migration mechanism of ethanolamine ammonia lyase $(E A L) ;{ }^{5}$ (C) Addition-elimination mechanism of 2-methyeneglutarate mutase (MGM). ${ }^{6}$

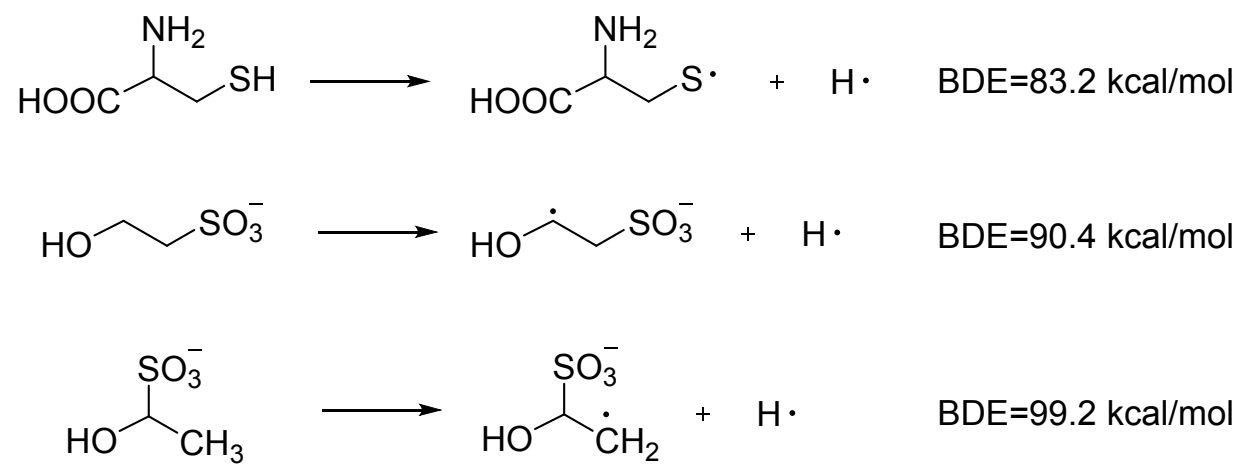

Figure S14. Bond dissociation energies of cysteine, isethionate and 1-hydroxyethane-1-sulfonate. All calculations were carried out at the B3LYP-D3/def2-TZVPP level. 


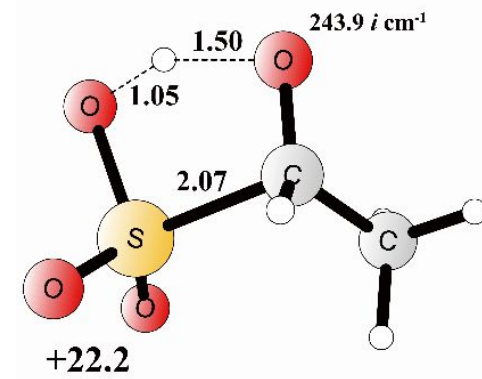

A

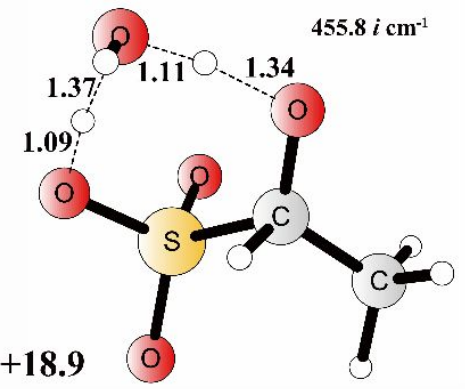

B

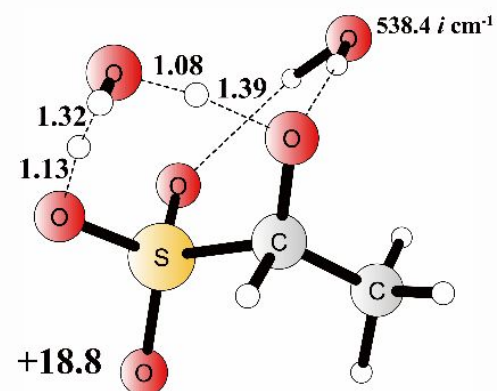

C

Figure S15. Optimized structures of TS6, TS6 $^{\prime}{ }_{w 1}$, TS6 $^{\prime}{ }_{w 2}$. Distances are given in Angstroms. (A) The transition state (TS6) for the direct elimination reaction. (B) The transition state (TS6' $\left.{ }^{\prime} \mathbf{1}\right)$ for the elimination reaction assisted by one water molecule in an aqueous solution. (C) The transition state (TS6' ${ }_{\text {w2 }}$ ) for the elimination reaction assisted by two water molecules in aqueous solution.

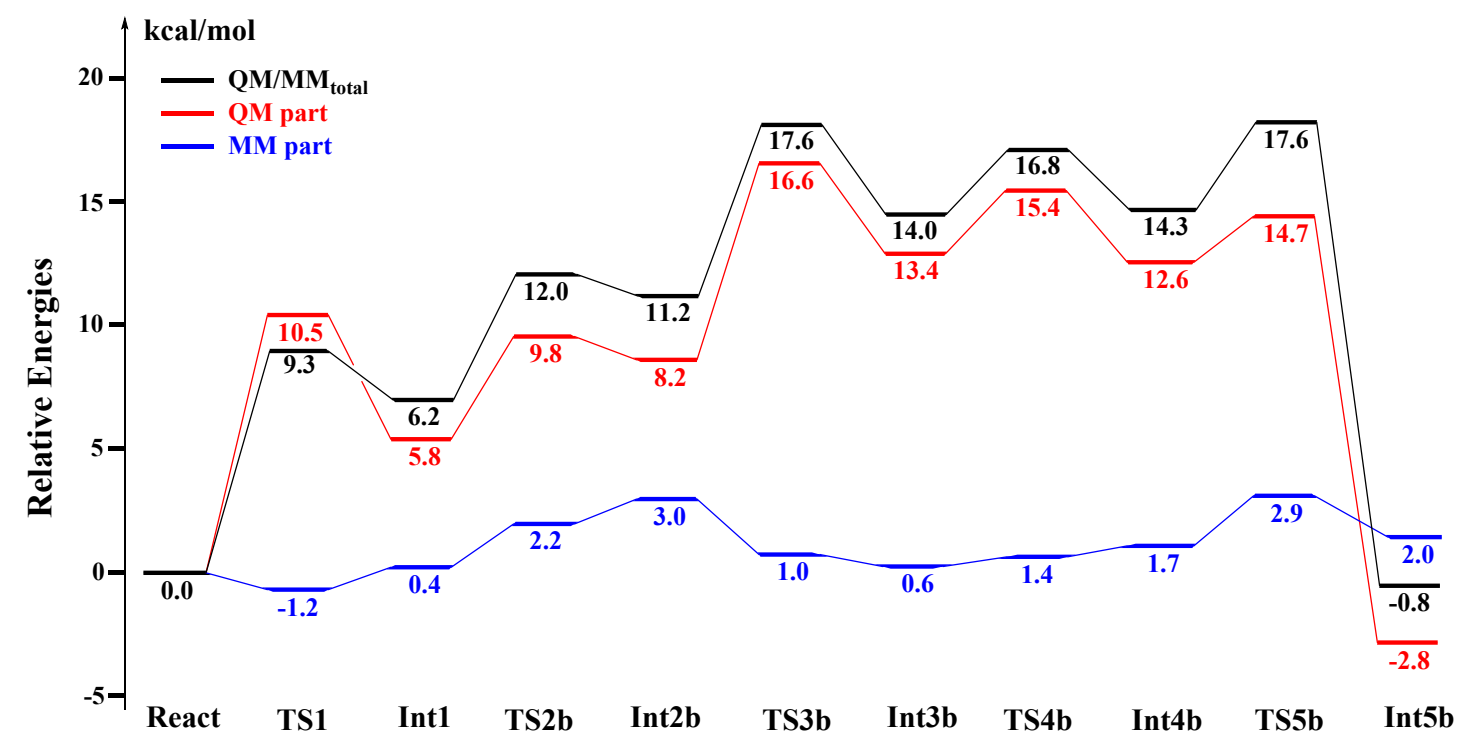

Figure S16. Electronic potential energy profile (in $\mathrm{kcal} / \mathrm{mol}$ ) calculated at the B3LYP-D3/def2-TZVPP:Charmm level. The energy profiles of QM part and MM part are highlighted using red line and blue line, repectively. 


\section{Cartesian coordinates for all optimized structures of QM}

\section{region}

\section{React:}

\begin{tabular}{|c|c|c|c|c|}
\hline \multirow{2}{*}{ Number } & \multirow{2}{*}{$\begin{array}{c}\text { Ato } \\
\text { m }\end{array}$} & \multicolumn{3}{|c|}{ Coordinates (Angstroms) } \\
\hline & & $\mathbf{X}$ & $\mathbf{Y}$ & $\mathbf{Z}$ \\
\hline 1 & $\mathrm{C}$ & -5.8944493 & 0.8225170 & -2.1688621 \\
\hline 2 & $\mathrm{H}$ & -5.5246662 & 1.7829007 & -1.7896014 \\
\hline 3 & $\mathrm{H}$ & -6.5144043 & 0.3656566 & -1.3797486 \\
\hline 4 & $\mathrm{~N}$ & -4.7635957 & -0.0193465 & -2.5474779 \\
\hline 5 & $\mathrm{H}$ & -4.7703122 & -0.4021022 & -3.4975051 \\
\hline 6 & $\mathrm{C}$ & -3.5917273 & -0.0266485 & -1.8921996 \\
\hline 7 & $\mathrm{~N}$ & -3.4358278 & 0.6604660 & -0.7557510 \\
\hline 8 & $\mathrm{H}$ & -2.5008967 & 0.7687389 & -0.3408810 \\
\hline 9 & $\mathrm{H}$ & -4.2066273 & 1.1493468 & -0.2914669 \\
\hline 10 & $\mathrm{~N}$ & -2.5576053 & -0.7228386 & -2.3845958 \\
\hline 11 & $\mathrm{H}$ & -1.6825263 & -0.8002949 & -1.8492073 \\
\hline 12 & $\mathrm{H}$ & -2.6829917 & -1.3378185 & -3.1988264 \\
\hline 13 & $\mathrm{C}$ & -1.5740891 & 4.6960023 & -1.1949664 \\
\hline 14 & 0 & -2.1503290 & 5.7778109 & -1.2314408 \\
\hline 15 & $\mathrm{~N}$ & -0.7603526 & 4.3466576 & -0.1683404 \\
\hline 16 & $\mathrm{H}$ & -0.4168494 & 3.3876201 & -0.1257486 \\
\hline 17 & $\mathrm{C}$ & -0.5217589 & 5.2356024 & 0.9674519 \\
\hline 18 & $\mathrm{H}$ & -1.4982082 & 5.6182464 & 1.3111856 \\
\hline 19 & $\mathrm{C}$ & 0.1408997 & 4.4728975 & 2.1282262 \\
\hline 20 & $\mathrm{H}$ & 0.4065161 & 5.1804997 & 2.9271906 \\
\hline 21 & $\mathrm{H}$ & -0.6324618 & 3.8153668 & 2.5569478 \\
\hline 22 & $\mathrm{C}$ & 1.3373138 & 3.5592643 & 1.8026124 \\
\hline 23 & $\mathrm{H}$ & 1.7528723 & 3.2135722 & 2.7625055 \\
\hline 24 & $\mathrm{H}$ & 1.0011343 & 2.6441798 & 1.3054801 \\
\hline 25 & $\mathrm{C}$ & 2.4805948 & 4.1924899 & 1.0326406 \\
\hline 26 & 0 & 2.8815241 & 5.3363730 & 1.2888397 \\
\hline 27 & $\mathrm{~N}$ & 3.0463867 & 3.4252605 & 0.0833840 \\
\hline 28 & $\mathrm{H}$ & 3.8482349 & 3.7772531 & -0.4446796 \\
\hline 29 & $\mathrm{H}$ & 2.6427133 & 2.5176748 & -0.1477647 \\
\hline 30 & $\mathrm{C}$ & 2.0939913 & 4.6608081 & 5.7080588 \\
\hline 31 & 0 & 2.6528853 & 3.5770395 & 5.5360279 \\
\hline 32 & $\mathrm{~N}$ & 0.7702263 & 4.7869821 & 5.8696685 \\
\hline 33 & $\mathrm{H}$ & 0.3588362 & 5.7214942 & 5.9873788 \\
\hline 34 & $\mathrm{C}$ & -0.0902763 & 3.6279487 & 5.7666687 \\
\hline 35 & $\mathrm{H}$ & -0.0630401 & 3.2126455 & 4.7469954 \\
\hline 36 & $\mathrm{H}$ & -1.1195948 & 3.9491258 & 5.9770088 \\
\hline & & & S21 & \\
\hline
\end{tabular}




\begin{tabular}{|c|c|c|c|c|}
\hline 37 & C & 0.2753836 & 2.5410854 & 6.7770825 \\
\hline 38 & 0 & 0.4437871 & 2.7878155 & 7.9714239 \\
\hline 39 & $\mathrm{~N}$ & 0.3438267 & 1.2995011 & 6.2627965 \\
\hline 40 & $\mathrm{H}$ & 0.2971055 & 1.1805181 & 5.2454437 \\
\hline 41 & $\mathrm{C}$ & 0.6158462 & 0.1635978 & 7.1141931 \\
\hline 42 & $\mathrm{H}$ & -0.1145492 & 0.1452393 & 7.9367887 \\
\hline 43 & C & 0.5190574 & -1.1444715 & 6.3175565 \\
\hline 44 & $\mathrm{H}$ & 1.2212362 & -1.1189940 & 5.4640898 \\
\hline 45 & $\mathrm{H}$ & 0.8665303 & -1.9860702 & 6.9374883 \\
\hline 46 & S & -1.1324144 & -1.5377716 & 5.6885476 \\
\hline 47 & $\mathrm{C}$ & 1.9774893 & 0.2669087 & 7.8365438 \\
\hline 48 & 0 & 2.0269893 & 0.2005620 & 9.0636723 \\
\hline 49 & $\mathrm{~N}$ & 3.0508141 & 0.3834545 & 7.0281152 \\
\hline 50 & $\mathrm{H}$ & 2.8980929 & 0.2647522 & 6.0175413 \\
\hline 51 & $\mathrm{C}$ & 4.4222812 & 0.3013090 & 7.5025483 \\
\hline 52 & $\mathrm{H}$ & 4.4917875 & 0.8814527 & 8.4356040 \\
\hline 53 & $\mathrm{C}$ & 4.8764737 & -1.1676592 & 7.7728002 \\
\hline 54 & $\mathrm{H}$ & 5.9761946 & -1.1626283 & 7.6771492 \\
\hline 55 & 0 & 4.2912006 & -2.0325575 & 6.8273380 \\
\hline 56 & $\mathrm{H}$ & 4.5020209 & -1.7348307 & 5.9219698 \\
\hline 57 & $\mathrm{C}$ & 4.5322275 & -1.6874689 & 9.1628729 \\
\hline 58 & $\mathrm{H}$ & 4.8559012 & -2.7393195 & 9.2412895 \\
\hline 59 & $\mathrm{H}$ & 5.0593350 & -1.1020726 & 9.9330479 \\
\hline 60 & $\mathrm{H}$ & 3.4553044 & -1.6044311 & 9.3611913 \\
\hline 61 & $\mathrm{C}$ & 5.4156517 & 0.9185388 & 6.4908231 \\
\hline 62 & 0 & 6.5989930 & 0.5941218 & 6.5008808 \\
\hline 63 & $\mathrm{~N}$ & 4.9070663 & 1.7881946 & 5.5873092 \\
\hline 64 & $\mathrm{H}$ & 3.9857412 & 2.2106482 & 5.7229845 \\
\hline 65 & $\mathrm{C}$ & 5.6477042 & 2.2132151 & 4.4137911 \\
\hline 66 & $\mathrm{H}$ & 6.3929379 & 1.4331670 & 4.1928905 \\
\hline 67 & $\mathrm{C}$ & 4.6861651 & 2.4256974 & 3.2329874 \\
\hline 68 & $\mathrm{H}$ & 3.8215368 & 3.0055085 & 3.5878447 \\
\hline 69 & $\mathrm{H}$ & 5.1558915 & 3.0161291 & 2.4284096 \\
\hline 70 & $\mathrm{C}$ & 4.2111189 & 1.1058511 & 2.5947634 \\
\hline 71 & $\mathrm{H}$ & 3.3331433 & 1.3070457 & 1.9630912 \\
\hline 72 & $\mathrm{H}$ & 5.0233050 & 0.7520475 & 1.9389596 \\
\hline 73 & $\mathrm{C}$ & 3.8672986 & -0.0149108 & 3.5917162 \\
\hline 74 & 0 & 2.7420589 & 0.0413906 & 4.1707919 \\
\hline 75 & 0 & 4.7229185 & -0.9204928 & 3.7719228 \\
\hline 76 & $\mathrm{C}$ & 4.8760418 & -4.5518602 & 1.6622491 \\
\hline 77 & $\mathrm{H}$ & 4.3143982 & -5.2066428 & 0.9883960 \\
\hline 78 & $\mathrm{H}$ & 4.9300253 & -5.0892787 & 2.6166189 \\
\hline 79 & $\mathrm{~N}$ & 4.1626437 & -3.2863625 & 1.8366025 \\
\hline 80 & $\mathrm{H}$ & 4.4614248 & -2.6612938 & 2.5883451 \\
\hline
\end{tabular}




\begin{tabular}{|c|c|c|c|c|}
\hline 81 & $\mathrm{C}$ & 3.4038483 & -2.7173763 & 0.8887130 \\
\hline 82 & $\mathrm{~N}$ & 2.7593690 & -3.4108419 & -0.0370793 \\
\hline 83 & $\mathrm{H}$ & 2.4211446 & -2.9214699 & -0.8693620 \\
\hline 84 & $\mathrm{H}$ & 2.6461886 & -4.4552342 & -0.0008275 \\
\hline 85 & $\mathrm{~N}$ & 3.3061203 & -1.3748877 & 0.9060777 \\
\hline 86 & $\mathrm{H}$ & 2.7297909 & -0.8258025 & 0.2646442 \\
\hline 87 & $\mathrm{H}$ & 4.0132934 & -0.8612583 & 1.4083892 \\
\hline 88 & $\mathrm{C}$ & -0.3715349 & 0.0041706 & 2.8689781 \\
\hline 89 & $\mathrm{C}$ & 0.1574813 & -0.8001687 & 1.6922181 \\
\hline 90 & $\mathrm{H}$ & 1.3781966 & 0.6768817 & 3.5530857 \\
\hline 91 & $\mathrm{H}$ & -0.5854544 & -0.7455371 & 3.6677751 \\
\hline 92 & $\mathrm{H}$ & -1.3392205 & 0.4523770 & 2.6026045 \\
\hline 93 & $\mathrm{H}$ & 1.1717143 & -1.1756716 & 1.8806488 \\
\hline 94 & $\mathrm{H}$ & -0.5019787 & -1.6561739 & 1.4891781 \\
\hline 95 & 0 & 0.4665618 & 1.0273619 & 3.3331203 \\
\hline 96 & 0 & -0.8231969 & 1.1897022 & 0.1987751 \\
\hline 97 & 0 & 1.6043427 & 0.6652257 & -0.0551326 \\
\hline 98 & 0 & -0.1130010 & -0.9103274 & -0.9394384 \\
\hline 99 & $\mathrm{~S}$ & 0.2204711 & 0.1149158 & 0.1141865 \\
\hline 100 & 0 & 1.8007075 & -2.0967952 & -2.5968535 \\
\hline 101 & $\mathrm{H}$ & 1.0433704 & -1.7644707 & -2.0716824 \\
\hline 102 & $\mathrm{H}$ & 1.6764362 & -3.0690677 & -2.7065490 \\
\hline 103 & $\mathrm{H}$ & -6.5060917 & 1.0574681 & -3.0399296 \\
\hline 104 & $\mathrm{H}$ & -1.6750978 & 3.9980890 & -2.0260991 \\
\hline 105 & $\mathrm{H}$ & 0.0070690 & 6.1404782 & 0.6681058 \\
\hline 106 & $\mathrm{H}$ & 2.6519728 & 5.5970829 & 5.7187030 \\
\hline 107 & $\mathrm{H}$ & 6.2181154 & 3.1154667 & 4.6343366 \\
\hline 108 & $\mathrm{H}$ & 5.8812312 & -4.3984445 & 1.2696616 \\
\hline
\end{tabular}




\begin{tabular}{|c|c|c|c|c|}
\hline \multirow{2}{*}{ Number } & \multirow{2}{*}{$\begin{array}{c}\text { Ato } \\
\text { m }\end{array}$} & \multicolumn{3}{|c|}{ Coordinates (Angstroms) } \\
\hline & & $\mathbf{X}$ & $\mathbf{Y}$ & $\mathbf{Z}$ \\
\hline 1 & $\mathrm{C}$ & -5.8899800 & 0.8365587 & -2.1710469 \\
\hline 2 & $\mathrm{H}$ & -5.5113240 & 1.8023585 & -1.8139368 \\
\hline 3 & $\mathrm{H}$ & -6.5052471 & 0.3972251 & -1.3683827 \\
\hline 4 & $\mathrm{~N}$ & -4.7665388 & -0.0181618 & -2.5422021 \\
\hline 5 & $\mathrm{H}$ & -4.7688587 & -0.4029706 & -3.4913516 \\
\hline 6 & $\mathrm{C}$ & -3.6052844 & -0.0400991 & -1.8693747 \\
\hline 7 & $\mathrm{~N}$ & -3.4598781 & 0.6479580 & -0.7326261 \\
\hline 8 & $\mathrm{H}$ & -2.5312804 & 0.7408713 & -0.3004405 \\
\hline 9 & $\mathrm{H}$ & -4.2331126 & 1.1406248 & -0.2766454 \\
\hline 10 & $\mathrm{~N}$ & -2.5703499 & -0.7488023 & -2.3443745 \\
\hline 11 & $\mathrm{H}$ & -1.7144075 & -0.8518229 & -1.7847394 \\
\hline 12 & $\mathrm{H}$ & -2.6893600 & -1.3610142 & -3.1617189 \\
\hline 13 & $\mathrm{C}$ & -1.5806353 & 4.6884609 & -1.1810178 \\
\hline 14 & 0 & -2.1521618 & 5.7722146 & -1.2194840 \\
\hline 15 & $\mathrm{~N}$ & -0.7670371 & 4.3384037 & -0.1538725 \\
\hline 16 & $\mathrm{H}$ & -0.4254790 & 3.3790462 & -0.1112655 \\
\hline 17 & $\mathrm{C}$ & -0.5195232 & 5.2280341 & 0.9794821 \\
\hline 18 & $\mathrm{H}$ & -1.4926241 & 5.6109619 & 1.3320765 \\
\hline 19 & C & 0.1529472 & 4.4637170 & 2.1336028 \\
\hline 20 & $\mathrm{H}$ & 0.4259602 & 5.1696910 & 2.9314862 \\
\hline 21 & $\mathrm{H}$ & -0.6175887 & 3.8069693 & 2.5692623 \\
\hline 22 & $\mathrm{C}$ & 1.3467735 & 3.5507058 & 1.7953609 \\
\hline 23 & $\mathrm{H}$ & 1.7669033 & 3.2006326 & 2.7515184 \\
\hline 24 & $\mathrm{H}$ & 1.0094117 & 2.6394952 & 1.2902046 \\
\hline 25 & $\mathrm{C}$ & 2.4896643 & 4.1861297 & 1.0261406 \\
\hline 26 & 0 & 2.8897162 & 5.3291640 & 1.2846521 \\
\hline 27 & $\mathrm{~N}$ & 3.0584504 & 3.4202100 & 0.0772019 \\
\hline 28 & $\mathrm{H}$ & 3.8636394 & 3.7702157 & -0.4481724 \\
\hline 29 & $\mathrm{H}$ & 2.6561804 & 2.5133797 & -0.1555662 \\
\hline 30 & $\mathrm{C}$ & 2.1038827 & 4.6599267 & 5.7109066 \\
\hline 31 & 0 & 2.6542445 & 3.5766148 & 5.5089683 \\
\hline 32 & $\mathrm{~N}$ & 0.7865402 & 4.7896906 & 5.9139310 \\
\hline 33 & $\mathrm{H}$ & 0.3805647 & 5.7261225 & 6.0363606 \\
\hline 34 & $\mathrm{C}$ & -0.0837693 & 3.6376324 & 5.8163738 \\
\hline 35 & $\mathrm{H}$ & -0.0762216 & 3.2352115 & 4.7915121 \\
\hline 36 & $\mathrm{H}$ & -1.1067388 & 3.9654011 & 6.0458589 \\
\hline 37 & $\mathrm{C}$ & 0.2857132 & 2.5339822 & 6.8100044 \\
\hline 38 & 0 & 0.4605743 & 2.7641454 & 8.0064506 \\
\hline 39 & $\mathrm{~N}$ & 0.3427132 & 1.3052603 & 6.2700591 \\
\hline 40 & $\mathrm{H}$ & 0.2878215 & 1.2182532 & 5.2540981 \\
\hline
\end{tabular}




\begin{tabular}{|c|c|c|c|c|}
\hline 41 & $\mathrm{C}$ & 0.5852482 & 0.1244493 & 7.0686609 \\
\hline 42 & $\mathrm{H}$ & -0.1630897 & 0.0732482 & 7.8727685 \\
\hline 43 & $\mathrm{C}$ & 0.4976714 & -1.1479715 & 6.2099737 \\
\hline 44 & $\mathrm{H}$ & 1.3008713 & -1.1525333 & 5.4573750 \\
\hline 45 & $\mathrm{H}$ & 0.6772709 & -2.0194776 & 6.8549968 \\
\hline 46 & $\mathrm{~S}$ & -1.1135180 & -1.4167107 & 5.3740133 \\
\hline 47 & $\mathrm{C}$ & 1.9352200 & 0.2009895 & 7.8183771 \\
\hline 48 & 0 & 1.9664868 & 0.1280673 & 9.0454544 \\
\hline 49 & $\mathrm{~N}$ & 3.0249796 & 0.3265578 & 7.0283468 \\
\hline 50 & $\mathrm{H}$ & 2.8895152 & 0.2170815 & 6.0159871 \\
\hline 51 & $\mathrm{C}$ & 4.3918160 & 0.2727716 & 7.5216017 \\
\hline 52 & $\mathrm{H}$ & 4.4411515 & 0.8689291 & 8.4466105 \\
\hline 53 & $\mathrm{C}$ & 4.8735907 & -1.1810693 & 7.8215151 \\
\hline 54 & $\mathrm{H}$ & 5.9743596 & -1.1514453 & 7.7472248 \\
\hline 55 & 0 & 4.3298890 & -2.0727591 & 6.8752816 \\
\hline 56 & $\mathrm{H}$ & 4.5413487 & -1.7710863 & 5.9726573 \\
\hline 57 & $\mathrm{C}$ & 4.5145626 & -1.6895087 & 9.2115283 \\
\hline 58 & $\mathrm{H}$ & 4.8508472 & -2.7359416 & 9.3073579 \\
\hline 59 & $\mathrm{H}$ & 5.0235656 & -1.0886020 & 9.9820196 \\
\hline 60 & $\mathrm{H}$ & 3.4342043 & -1.6159461 & 9.3925409 \\
\hline 61 & $\mathrm{C}$ & 5.3894390 & 0.8920133 & 6.5125120 \\
\hline 62 & 0 & 6.5735015 & 0.5704424 & 6.5284931 \\
\hline 63 & $\mathrm{~N}$ & 4.8887197 & 1.7619175 & 5.6036568 \\
\hline 64 & $\mathrm{H}$ & 3.9676099 & 2.1870682 & 5.7309426 \\
\hline 65 & $\mathrm{C}$ & 5.6520635 & 2.1959714 & 4.4473227 \\
\hline 66 & $\mathrm{H}$ & 6.3974290 & 1.4145927 & 4.2322707 \\
\hline 67 & $\mathrm{C}$ & 4.7171507 & 2.4267638 & 3.2482056 \\
\hline 68 & $\mathrm{H}$ & 3.8482244 & 3.0077411 & 3.5904693 \\
\hline 69 & $\mathrm{H}$ & 5.2080413 & 3.0229337 & 2.4605075 \\
\hline 70 & $\mathrm{C}$ & 4.2503670 & 1.1163828 & 2.5845189 \\
\hline 71 & $\mathrm{H}$ & 3.3815426 & 1.3257134 & 1.9429747 \\
\hline 72 & $\mathrm{H}$ & 5.0700839 & 0.7658697 & 1.9369551 \\
\hline 73 & $\mathrm{C}$ & 3.8913903 & -0.0100596 & 3.5660816 \\
\hline 74 & 0 & 2.7545747 & 0.0424389 & 4.1278861 \\
\hline 75 & 0 & 4.7386324 & -0.9175441 & 3.7566570 \\
\hline 76 & $\mathrm{C}$ & 4.8728126 & -4.5663232 & 1.6588324 \\
\hline 77 & $\mathrm{H}$ & 4.3138499 & -5.2151597 & 0.9770343 \\
\hline 78 & $\mathrm{H}$ & 4.9257642 & -5.1131442 & 2.6079982 \\
\hline 79 & $\mathrm{~N}$ & 4.1573742 & -3.3040480 & 1.8430257 \\
\hline 80 & $\mathrm{H}$ & 4.4581921 & -2.6815543 & 2.5955555 \\
\hline 81 & $\mathrm{C}$ & 3.3812406 & -2.7369473 & 0.9087086 \\
\hline 82 & $\mathrm{~N}$ & 2.7306783 & -3.4300683 & -0.0124850 \\
\hline 83 & $\mathrm{H}$ & 2.3790049 & -2.9380086 & -0.8365226 \\
\hline \multirow[t]{2}{*}{84} & $\mathrm{H}$ & 2.6244833 & -4.4756378 & 0.0178560 \\
\hline & & & S25 & \\
\hline
\end{tabular}




\begin{tabular}{ccccc}
85 & $\mathrm{~N}$ & 3.2741116 & -1.3953336 & 0.9338541 \\
86 & $\mathrm{H}$ & 2.6815407 & -0.8475930 & 0.3057437 \\
87 & $\mathrm{H}$ & 3.9920074 & -0.8812694 & 1.4199384 \\
88 & $\mathrm{C}$ & -0.3283140 & 0.1403686 & 2.9317604 \\
89 & $\mathrm{C}$ & 0.1247411 & -0.7803089 & 1.8442994 \\
90 & $\mathrm{H}$ & 1.4722094 & 0.6962090 & 3.5817473 \\
91 & $\mathrm{H}$ & -0.7162587 & -0.7274308 & 4.0666115 \\
92 & $\mathrm{H}$ & -1.3236529 & 0.5671802 & 2.7624073 \\
93 & $\mathrm{H}$ & 1.1301408 & -1.1857614 & 2.0214400 \\
94 & $\mathrm{H}$ & -0.5841539 & -1.6081150 & 1.7034352 \\
95 & $\mathrm{O}$ & 0.5400242 & 1.0726523 & 3.4038318 \\
96 & $\mathrm{O}$ & -0.8581474 & 1.1424360 & 0.2402083 \\
97 & $\mathrm{O}$ & 1.5620145 & 0.6114588 & -0.0058265 \\
98 & $\mathrm{O}$ & -0.1601915 & -1.0098225 & -0.8118691 \\
99 & $\mathrm{~S}$ & 0.1811672 & 0.0609339 & 0.1912896 \\
100 & $\mathrm{O}$ & 1.7508783 & -2.0993663 & -2.5686647 \\
101 & $\mathrm{H}$ & 0.9976198 & -1.7946443 & -2.0224350 \\
102 & $\mathrm{H}$ & 1.6479890 & -3.0737841 & -2.6832215 \\
103 & $\mathrm{H}$ & -6.5093115 & 1.0594756 & -3.0398413 \\
104 & $\mathrm{H}$ & -1.6836893 & 3.9881356 & -2.0098673 \\
105 & $\mathrm{H}$ & 0.0085801 & 6.1322776 & 0.6769628 \\
106 & $\mathrm{H}$ & 2.6654600 & 5.5940880 & 5.7172839 \\
107 & $\mathrm{H}$ & 6.2256503 & 3.0919837 & 4.6844457 \\
108 & $\mathrm{H}$ & 5.8778428 & -4.4057815 & 1.2686944 \\
\hline & & & & \\
\hline
\end{tabular}


Int1

\begin{tabular}{|c|c|c|c|c|}
\hline \multirow{2}{*}{ Number } & \multirow{2}{*}{$\begin{array}{c}\text { Ato } \\
\text { m }\end{array}$} & \multicolumn{3}{|c|}{ Coordinates (Angstroms) } \\
\hline & & $\mathbf{X}$ & $\mathbf{Y}$ & $\mathbf{Z}$ \\
\hline 1 & $\mathrm{C}$ & -5.8744378 & 0.8375294 & -2.1733927 \\
\hline 2 & $\mathrm{H}$ & -5.4942039 & 1.8043203 & -1.8205749 \\
\hline 3 & $\mathrm{H}$ & -6.4852001 & 0.3995877 & -1.3665328 \\
\hline 4 & $\mathrm{~N}$ & -4.7523362 & -0.0171946 & -2.5491172 \\
\hline 5 & $\mathrm{H}$ & -4.7600044 & -0.4013915 & -3.4984752 \\
\hline 6 & $\mathrm{C}$ & -3.5839748 & -0.0335014 & -1.8879073 \\
\hline 7 & $\mathrm{~N}$ & -3.4308267 & 0.6509755 & -0.7498399 \\
\hline 8 & $\mathrm{H}$ & -2.4941794 & 0.7596649 & -0.3384571 \\
\hline 9 & $\mathrm{H}$ & -4.2008469 & 1.1439082 & -0.2892534 \\
\hline 10 & $\mathrm{~N}$ & -2.5501798 & -0.7343747 & -2.3759025 \\
\hline 11 & $\mathrm{H}$ & -1.6863294 & -0.8336000 & -1.8270475 \\
\hline 12 & $\mathrm{H}$ & -2.6761445 & -1.3490872 & -3.1904197 \\
\hline 13 & $\mathrm{C}$ & -1.5695988 & 4.6977583 & -1.2065618 \\
\hline 14 & 0 & -2.1422298 & 5.7813829 & -1.2414622 \\
\hline 15 & $\mathrm{~N}$ & -0.7586511 & 4.3426089 & -0.1796529 \\
\hline 16 & $\mathrm{H}$ & -0.4194867 & 3.3815506 & -0.1427191 \\
\hline 17 & $\mathrm{C}$ & -0.5163017 & 5.2259229 & 0.9598307 \\
\hline 18 & $\mathrm{H}$ & -1.4908389 & 5.6062916 & 1.3115471 \\
\hline 19 & $\mathrm{C}$ & 0.1531323 & 4.4549296 & 2.1112277 \\
\hline 20 & $\mathrm{H}$ & 0.4254851 & 5.1555565 & 2.9140271 \\
\hline 21 & $\mathrm{H}$ & -0.6186238 & 3.7962991 & 2.5420276 \\
\hline 22 & $\mathrm{C}$ & 1.3470435 & 3.5438686 & 1.7683019 \\
\hline 23 & $\mathrm{H}$ & 1.7629647 & 3.1827468 & 2.7219737 \\
\hline 24 & $\mathrm{H}$ & 1.0095593 & 2.6405890 & 1.2490138 \\
\hline 25 & $\mathrm{C}$ & 2.4930345 & 4.1858203 & 1.0081673 \\
\hline 26 & 0 & 2.8921116 & 5.3263362 & 1.2800462 \\
\hline 27 & $\mathrm{~N}$ & 3.0637738 & 3.4282914 & 0.0541374 \\
\hline 28 & $\mathrm{H}$ & 3.8713437 & 3.7825712 & -0.4650707 \\
\hline 29 & $\mathrm{H}$ & 2.6606391 & 2.5239814 & -0.1903945 \\
\hline 30 & $\mathrm{C}$ & 2.0928595 & 4.6536502 & 5.7085764 \\
\hline 31 & 0 & 2.6466995 & 3.5702601 & 5.5164730 \\
\hline 32 & $\mathrm{~N}$ & 0.7729512 & 4.7800124 & 5.8983148 \\
\hline 33 & $\mathrm{H}$ & 0.3635625 & 5.7149229 & 6.0200985 \\
\hline 34 & $\mathrm{C}$ & -0.0905366 & 3.6220622 & 5.8060041 \\
\hline 35 & $\mathrm{H}$ & -0.0718739 & 3.2072362 & 4.7864412 \\
\hline 36 & $\mathrm{H}$ & -1.1170006 & 3.9467960 & 6.0241062 \\
\hline 37 & $\mathrm{C}$ & 0.2812389 & 2.5346242 & 6.8161794 \\
\hline 38 & 0 & 0.4696760 & 2.7878594 & 8.0056862 \\
\hline 39 & $\mathrm{~N}$ & 0.3301066 & 1.2917827 & 6.3050649 \\
\hline 40 & $\mathrm{H}$ & 0.2695331 & 1.1762401 & 5.2918185 \\
\hline
\end{tabular}




\begin{tabular}{|c|c|c|c|c|}
\hline 41 & $\mathrm{C}$ & 0.6151407 & 0.1434594 & 7.1355705 \\
\hline 42 & $\mathrm{H}$ & -0.1118708 & 0.1032840 & 7.9603954 \\
\hline 43 & $\mathrm{C}$ & 0.5387954 & -1.1590321 & 6.3252422 \\
\hline 44 & $\mathrm{H}$ & 1.2810510 & -1.1483798 & 5.5135896 \\
\hline 45 & $\mathrm{H}$ & 0.8129398 & -1.9938719 & 6.9836083 \\
\hline 46 & $\mathrm{~S}$ & -1.1184041 & -1.5511992 & 5.6381864 \\
\hline 47 & $\mathrm{C}$ & 1.9809542 & 0.2571209 & 7.8478600 \\
\hline 48 & 0 & 2.0435926 & 0.1969560 & 9.0739904 \\
\hline 49 & $\mathrm{~N}$ & 3.0502109 & 0.3789139 & 7.0309869 \\
\hline 50 & $\mathrm{H}$ & 2.8937632 & 0.2547549 & 6.0235934 \\
\hline 51 & $\mathrm{C}$ & 4.4243883 & 0.3028861 & 7.4993276 \\
\hline 52 & $\mathrm{H}$ & 4.4953499 & 0.8855739 & 8.4307070 \\
\hline 53 & $\mathrm{C}$ & 4.8871930 & -1.1628816 & 7.7716932 \\
\hline 54 & $\mathrm{H}$ & 5.9875456 & -1.1489982 & 7.6841794 \\
\hline 55 & 0 & 4.3160113 & -2.0303639 & 6.8200104 \\
\hline 56 & $\mathrm{H}$ & 4.5303103 & -1.7288053 & 5.9173156 \\
\hline 57 & $\mathrm{C}$ & 4.5374863 & -1.6892217 & 9.1578367 \\
\hline 58 & $\mathrm{H}$ & 4.8718785 & -2.7376419 & 9.2362969 \\
\hline 59 & $\mathrm{H}$ & 5.0517372 & -1.0993484 & 9.9332214 \\
\hline 60 & $\mathrm{H}$ & 3.4584916 & -1.6189747 & 9.3483603 \\
\hline 61 & $\mathrm{C}$ & 5.4136542 & 0.9208766 & 6.4840991 \\
\hline 62 & 0 & 6.5982317 & 0.6010521 & 6.4941650 \\
\hline 63 & $\mathrm{~N}$ & 4.9032389 & 1.7864181 & 5.5764487 \\
\hline 64 & $\mathrm{H}$ & 3.9832368 & 2.2118479 & 5.7125541 \\
\hline 65 & $\mathrm{C}$ & 5.6521276 & 2.2167123 & 4.4098836 \\
\hline 66 & $\mathrm{H}$ & 6.3981828 & 1.4370624 & 4.1905449 \\
\hline 67 & $\mathrm{C}$ & 4.7021568 & 2.4381094 & 3.2210977 \\
\hline 68 & $\mathrm{H}$ & 3.8348945 & 3.0172969 & 3.5710352 \\
\hline 69 & $\mathrm{H}$ & 5.1806387 & 3.0324562 & 2.4246066 \\
\hline 70 & $\mathrm{C}$ & 4.2330252 & 1.1222935 & 2.5698526 \\
\hline 71 & $\mathrm{H}$ & 3.3623205 & 1.3258895 & 1.9289606 \\
\hline 72 & $\mathrm{H}$ & 5.0505637 & 0.7688663 & 1.9210383 \\
\hline 73 & $\mathrm{C}$ & 3.8828135 & 0.0012690 & 3.5607643 \\
\hline 74 & 0 & 2.7477748 & 0.0507022 & 4.1276684 \\
\hline 75 & 0 & 4.7362180 & -0.8991515 & 3.7564247 \\
\hline 76 & $\mathrm{C}$ & 4.8759907 & -4.5544563 & 1.6627617 \\
\hline 77 & $\mathrm{H}$ & 4.3154970 & -5.2113748 & 0.9899647 \\
\hline 78 & $\mathrm{H}$ & 4.9302830 & -5.0893984 & 2.6185167 \\
\hline 79 & $\mathrm{~N}$ & 4.1610191 & -3.2893531 & 1.8335596 \\
\hline 80 & $\mathrm{H}$ & 4.4594254 & -2.6620161 & 2.5828828 \\
\hline 81 & $\mathrm{C}$ & 3.3928651 & -2.7273168 & 0.8894632 \\
\hline 82 & $\mathrm{~N}$ & 2.7491861 & -3.4248771 & -0.0325222 \\
\hline 83 & $\mathrm{H}$ & 2.4011077 & -2.9372332 & -0.8612737 \\
\hline \multirow[t]{2}{*}{84} & $\mathrm{H}$ & 2.6407398 & -4.4701440 & 0.0035256 \\
\hline & & & S28 & \\
\hline
\end{tabular}




\begin{tabular}{ccccc}
85 & $\mathrm{~N}$ & 3.2836893 & -1.3841975 & 0.9077095 \\
86 & $\mathrm{H}$ & 2.7175925 & -0.8441368 & 0.2491234 \\
87 & $\mathrm{H}$ & 4.0105638 & -0.8676148 & 1.3782661 \\
88 & $\mathrm{C}$ & -0.3003189 & 0.2291675 & 2.8249777 \\
89 & $\mathrm{C}$ & 0.1708673 & -0.7059059 & 1.7921587 \\
90 & $\mathrm{H}$ & 1.4756187 & 0.7062734 & 3.6005750 \\
91 & $\mathrm{H}$ & -0.9379175 & -0.9088670 & 4.4464792 \\
92 & $\mathrm{H}$ & -1.3287605 & 0.5891218 & 2.7581850 \\
93 & $\mathrm{H}$ & 1.1812060 & -1.0931227 & 1.9808898 \\
94 & $\mathrm{H}$ & -0.5274657 & -1.5418782 & 1.6444055 \\
95 & $\mathrm{O}$ & 0.5359259 & 1.0840080 & 3.4468217 \\
96 & $\mathrm{O}$ & -0.8050793 & 1.1814384 & 0.1329205 \\
97 & $\mathrm{O}$ & 1.6169522 & 0.6426746 & -0.1139502 \\
98 & $\mathrm{O}$ & -0.1168535 & -0.9882955 & -0.8897324 \\
99 & $\mathrm{~S}$ & 0.2338511 & 0.0968780 & 0.0971974 \\
100 & $\mathrm{O}$ & 1.7921156 & -2.1142257 & -2.6000163 \\
101 & $\mathrm{H}$ & 1.0340042 & -1.7971171 & -2.0670218 \\
102 & $\mathrm{H}$ & 1.6805797 & -3.0881093 & -2.7112728 \\
103 & $\mathrm{H}$ & -6.4982032 & 1.0580598 & -3.0396203 \\
104 & $\mathrm{H}$ & -1.6704904 & 3.9999490 & -2.0377959 \\
105 & $\mathrm{H}$ & 0.0118338 & 6.1322730 & 0.6637402 \\
106 & $\mathrm{H}$ & 2.6519768 & 5.5892771 & 5.7161494 \\
107 & $\mathrm{H}$ & 6.2230034 & 3.1168854 & 4.6376037 \\
108 & $\mathrm{H}$ & 5.8810805 & -4.3999870 & 1.2703324 \\
\hline & & & & \\
\hline
\end{tabular}


TS2b

\begin{tabular}{|c|c|c|c|c|}
\hline \multirow{2}{*}{ Number } & \multirow{2}{*}{$\begin{array}{c}\text { Ato } \\
\text { m }\end{array}$} & \multicolumn{3}{|c|}{ Coordinates (Angstroms) } \\
\hline & & $\mathbf{X}$ & $\mathbf{Y}$ & $\mathbf{Z}$ \\
\hline 1 & $\mathrm{C}$ & -5.8812020 & 0.8276253 & -2.1646045 \\
\hline 2 & $\mathrm{H}$ & -5.5004780 & 1.7901821 & -1.8018978 \\
\hline 3 & $\mathrm{H}$ & -6.4959921 & 0.3852458 & -1.3631843 \\
\hline 4 & $\mathrm{~N}$ & -4.7610332 & -0.0262918 & -2.5457079 \\
\hline 5 & $\mathrm{H}$ & -4.7775836 & -0.4090111 & -3.4953410 \\
\hline 6 & $\mathrm{C}$ & -3.5782845 & -0.0366039 & -1.9067883 \\
\hline 7 & $\mathrm{~N}$ & -3.4006464 & 0.6534737 & -0.7744039 \\
\hline 8 & $\mathrm{H}$ & -2.4592449 & 0.7470417 & -0.3659785 \\
\hline 9 & $\mathrm{H}$ & -4.1641888 & 1.1453565 & -0.3025153 \\
\hline 10 & $\mathrm{~N}$ & -2.5575576 & -0.7376487 & -2.4137877 \\
\hline 11 & $\mathrm{H}$ & -1.6697910 & -0.8160687 & -1.8936303 \\
\hline 12 & $\mathrm{H}$ & -2.6970756 & -1.3508700 & -3.2269982 \\
\hline 13 & $\mathrm{C}$ & -1.5763963 & 4.6854400 & -1.1982730 \\
\hline 14 & 0 & -2.1367483 & 5.7763073 & -1.2305317 \\
\hline 15 & $\mathrm{~N}$ & -0.7722844 & 4.3172828 & -0.1705321 \\
\hline 16 & $\mathrm{H}$ & -0.4458557 & 3.3501290 & -0.1346085 \\
\hline 17 & $\mathrm{C}$ & -0.5250705 & 5.1988270 & 0.9702652 \\
\hline 18 & $\mathrm{H}$ & -1.4979123 & 5.5888970 & 1.3167045 \\
\hline 19 & $\mathrm{C}$ & 0.1273589 & 4.4210265 & 2.1277758 \\
\hline 20 & $\mathrm{H}$ & 0.4097425 & 5.1214887 & 2.9272122 \\
\hline 21 & $\mathrm{H}$ & -0.6580084 & 3.7787524 & 2.5581024 \\
\hline 22 & $\mathrm{C}$ & 1.3074618 & 3.4870490 & 1.7939982 \\
\hline 23 & $\mathrm{H}$ & 1.7257389 & 3.1353395 & 2.7513097 \\
\hline 24 & $\mathrm{H}$ & 0.9522717 & 2.5847731 & 1.2834168 \\
\hline 25 & $\mathrm{C}$ & 2.4530478 & 4.1144550 & 1.0168615 \\
\hline 26 & 0 & 2.8825736 & 5.2433995 & 1.2951711 \\
\hline 27 & $\mathrm{~N}$ & 2.9794352 & 3.3610826 & 0.0366545 \\
\hline 28 & $\mathrm{H}$ & 3.7724214 & 3.7195666 & -0.4988530 \\
\hline 29 & $\mathrm{H}$ & 2.5591629 & 2.4616665 & -0.2119686 \\
\hline 30 & $\mathrm{C}$ & 2.0727288 & 4.6374550 & 5.7225690 \\
\hline 31 & 0 & 2.6137975 & 3.5473974 & 5.5324261 \\
\hline 32 & $\mathrm{~N}$ & 0.7540012 & 4.7728472 & 5.9189343 \\
\hline 33 & $\mathrm{H}$ & 0.3498737 & 5.7110399 & 6.0352350 \\
\hline 34 & $\mathrm{C}$ & -0.1170159 & 3.6212051 & 5.8318314 \\
\hline 35 & $\mathrm{H}$ & -0.1061920 & 3.2022461 & 4.8133254 \\
\hline 36 & $\mathrm{H}$ & -1.1405580 & 3.9555828 & 6.0488879 \\
\hline 37 & $\mathrm{C}$ & 0.2491677 & 2.5348524 & 6.8453041 \\
\hline 38 & 0 & 0.4647230 & 2.7888684 & 8.0285607 \\
\hline 39 & $\mathrm{~N}$ & 0.2613119 & 1.2824827 & 6.3455157 \\
\hline 40 & $\mathrm{H}$ & 0.2117194 & 1.1551975 & 5.3305074 \\
\hline
\end{tabular}




\begin{tabular}{|c|c|c|c|c|}
\hline 41 & $\mathrm{C}$ & 0.6159613 & 0.1501464 & 7.1739625 \\
\hline 42 & $\mathrm{H}$ & -0.0843831 & 0.0785981 & 8.0211565 \\
\hline 43 & $\mathrm{C}$ & 0.5880233 & -1.1484484 & 6.3654751 \\
\hline 44 & $\mathrm{H}$ & 1.2619533 & -1.0659277 & 5.5004439 \\
\hline 45 & $\mathrm{H}$ & 0.9730837 & -1.9621898 & 6.9925213 \\
\hline 46 & $\mathrm{~S}$ & -1.0818018 & -1.6404566 & 5.7392041 \\
\hline 47 & $\mathrm{C}$ & 1.9945740 & 0.3039529 & 7.8599073 \\
\hline 48 & 0 & 2.0742223 & 0.2293499 & 9.0831919 \\
\hline 49 & $\mathrm{~N}$ & 3.0432719 & 0.4622263 & 7.0267866 \\
\hline 50 & $\mathrm{H}$ & 2.8716519 & 0.3479644 & 6.0197697 \\
\hline 51 & $\mathrm{C}$ & 4.4232729 & 0.3413642 & 7.4706228 \\
\hline 52 & $\mathrm{H}$ & 4.5228152 & 0.8904461 & 8.4187931 \\
\hline 53 & $\mathrm{C}$ & 4.8425946 & -1.1508089 & 7.6862790 \\
\hline 54 & $\mathrm{H}$ & 5.9351642 & -1.1787441 & 7.5338024 \\
\hline 55 & 0 & 4.1794459 & -1.9709107 & 6.7530112 \\
\hline 56 & $\mathrm{H}$ & 4.3938730 & -1.6850627 & 5.8435259 \\
\hline 57 & $\mathrm{C}$ & 4.5554234 & -1.6905220 & 9.0819285 \\
\hline 58 & $\mathrm{H}$ & 4.8598554 & -2.7498886 & 9.1253743 \\
\hline 59 & $\mathrm{H}$ & 5.1323698 & -1.1307641 & 9.8352247 \\
\hline 60 & $\mathrm{H}$ & 3.4914851 & -1.5919101 & 9.3353813 \\
\hline 61 & $\mathrm{C}$ & 5.4159348 & 0.9617116 & 6.4635669 \\
\hline 62 & 0 & 6.5990534 & 0.6371574 & 6.4803219 \\
\hline 63 & $\mathrm{~N}$ & 4.9092554 & 1.8285847 & 5.5556121 \\
\hline 64 & $\mathrm{H}$ & 3.9899575 & 2.2568319 & 5.6904481 \\
\hline 65 & $\mathrm{C}$ & 5.6531022 & 2.2462338 & 4.3815104 \\
\hline 66 & $\mathrm{H}$ & 6.3964119 & 1.4631116 & 4.1651904 \\
\hline 67 & $\mathrm{C}$ & 4.6985711 & 2.4596663 & 3.1948160 \\
\hline 68 & $\mathrm{H}$ & 3.8318244 & 3.0417353 & 3.5415449 \\
\hline 69 & $\mathrm{H}$ & 5.1738087 & 3.0473897 & 2.3917665 \\
\hline 70 & $\mathrm{C}$ & 4.2270710 & 1.1379384 & 2.5584054 \\
\hline 71 & $\mathrm{H}$ & 3.3647322 & 1.3355075 & 1.9050766 \\
\hline 72 & $\mathrm{H}$ & 5.0490469 & 0.7698751 & 1.9236481 \\
\hline 73 & $\mathrm{C}$ & 3.8595009 & 0.0422180 & 3.5677950 \\
\hline 74 & 0 & 2.7279285 & 0.1296672 & 4.1406892 \\
\hline 75 & 0 & 4.6955779 & -0.8689620 & 3.7851636 \\
\hline 76 & $\mathrm{C}$ & 4.8621875 & -4.5408881 & 1.6205792 \\
\hline 77 & $\mathrm{H}$ & 4.3089995 & -5.1756324 & 0.9219864 \\
\hline 78 & $\mathrm{H}$ & 4.8892736 & -5.1004794 & 2.5631998 \\
\hline 79 & $\mathrm{~N}$ & 4.1561309 & -3.2721617 & 1.8000507 \\
\hline 80 & $\mathrm{H}$ & 4.4616681 & -2.6509248 & 2.5500090 \\
\hline 81 & $\mathrm{C}$ & 3.3881827 & -2.6982231 & 0.8603006 \\
\hline 82 & $\mathrm{~N}$ & 2.7304958 & -3.3895135 & -0.0564200 \\
\hline 83 & $\mathrm{H}$ & 2.3938082 & -2.9019232 & -0.8923096 \\
\hline 84 & $\mathrm{H}$ & 2.6192285 & -4.4338682 & -0.0175618 \\
\hline
\end{tabular}




\begin{tabular}{ccccc}
85 & $\mathrm{~N}$ & 3.2938829 & -1.3544558 & 0.8848085 \\
86 & $\mathrm{H}$ & 2.7270938 & -0.7993986 & 0.2337222 \\
87 & $\mathrm{H}$ & 4.0340786 & -0.8502631 & 1.3477982 \\
88 & $\mathrm{C}$ & -0.3142498 & -0.0094207 & 2.9187786 \\
89 & $\mathrm{C}$ & 0.2310955 & -0.9652250 & 2.0713883 \\
90 & $\mathrm{H}$ & 1.3964446 & 0.6796619 & 3.6348558 \\
91 & $\mathrm{H}$ & -1.7784732 & -0.5963614 & 6.2354588 \\
92 & $\mathrm{H}$ & -1.3942289 & 0.1234267 & 3.0093091 \\
93 & $\mathrm{H}$ & 1.3003551 & -1.1771397 & 2.1465794 \\
94 & $\mathrm{H}$ & -0.4042117 & -1.7976508 & 1.7585680 \\
95 & $\mathrm{O}$ & 0.4052510 & 0.9272636 & 3.5445928 \\
96 & $\mathrm{O}$ & -0.7558077 & 1.1628360 & 0.1491355 \\
97 & $\mathrm{O}$ & 1.6638653 & 0.6421091 & -0.2518702 \\
98 & $\mathrm{O}$ & -0.1206615 & -0.9363560 & -1.0624196 \\
99 & $\mathrm{~S}$ & 0.2819169 & 0.0647889 & 0.0145735 \\
100 & $\mathrm{O}$ & 1.8255966 & -2.1255314 & -2.6313262 \\
101 & $\mathrm{H}$ & 1.0525926 & -1.7816168 & -2.1333059 \\
102 & $\mathrm{H}$ & 1.6943907 & -3.0965727 & -2.7430064 \\
103 & $\mathrm{H}$ & -6.5004911 & 1.0553396 & -3.0321841 \\
104 & $\mathrm{H}$ & -1.6839077 & 3.9933748 & -2.0334701 \\
105 & $\mathrm{H}$ & 0.0109104 & 6.1013017 & 0.6764438 \\
106 & $\mathrm{H}$ & 2.6373610 & 5.5697938 & 5.7239570 \\
107 & $\mathrm{H}$ & 6.2256899 & 3.1473017 & 4.6012538 \\
108 & $\mathrm{H}$ & 5.8763577 & -4.3925587 & 1.2497363 \\
\hline & & & &
\end{tabular}


Int2a

\begin{tabular}{|c|c|c|c|c|}
\hline \multirow{2}{*}{ Number } & \multirow{2}{*}{$\begin{array}{c}\text { Ato } \\
\text { m }\end{array}$} & \multicolumn{3}{|c|}{ Coordinates (Angstroms) } \\
\hline & & $\mathbf{X}$ & $\mathbf{Y}$ & $\mathbf{Z}$ \\
\hline 1 & $\mathrm{C}$ & -5.9074258 & 0.8160869 & -2.1387659 \\
\hline 2 & $\mathrm{H}$ & -5.5276740 & 1.7720332 & -1.7585772 \\
\hline 3 & $\mathrm{H}$ & -6.5335626 & 0.3676897 & -1.3495139 \\
\hline 4 & $\mathrm{~N}$ & -4.7881241 & -0.0380980 & -2.5200257 \\
\hline 5 & $\mathrm{H}$ & -4.8080652 & -0.4147982 & -3.4712772 \\
\hline 6 & $\mathrm{C}$ & -3.5906524 & -0.0332833 & -1.9036536 \\
\hline 7 & $\mathrm{~N}$ & -3.3956889 & 0.6604654 & -0.7762387 \\
\hline 8 & $\mathrm{H}$ & -2.4469616 & 0.7650491 & -0.3838778 \\
\hline 9 & $\mathrm{H}$ & -4.1556083 & 1.1468318 & -0.2933911 \\
\hline 10 & $\mathrm{~N}$ & -2.5788429 & -0.7274594 & -2.4306771 \\
\hline 11 & $\mathrm{H}$ & -1.6565134 & -0.7534844 & -1.9599409 \\
\hline 12 & $\mathrm{H}$ & -2.7261154 & -1.3328887 & -3.2475560 \\
\hline 13 & $\mathrm{C}$ & -1.5668242 & 4.6909499 & -1.2096618 \\
\hline 14 & 0 & -2.1141169 & 5.7894915 & -1.2490527 \\
\hline 15 & $\mathrm{~N}$ & -0.7731538 & 4.3147894 & -0.1776707 \\
\hline 16 & $\mathrm{H}$ & -0.4757664 & 3.3356408 & -0.1297290 \\
\hline 17 & $\mathrm{C}$ & -0.5282980 & 5.1979015 & 0.9628972 \\
\hline 18 & $\mathrm{H}$ & -1.5010351 & 5.5943375 & 1.3034881 \\
\hline 19 & $\mathrm{C}$ & 0.1157977 & 4.4230976 & 2.1265598 \\
\hline 20 & $\mathrm{H}$ & 0.3965070 & 5.1263587 & 2.9245587 \\
\hline 21 & $\mathrm{H}$ & -0.6729782 & 3.7841770 & 2.5552753 \\
\hline 22 & $\mathrm{C}$ & 1.2927207 & 3.4834816 & 1.8012657 \\
\hline 23 & $\mathrm{H}$ & 1.6969034 & 3.1244987 & 2.7615051 \\
\hline 24 & $\mathrm{H}$ & 0.9332824 & 2.5828192 & 1.2918891 \\
\hline 25 & $\mathrm{C}$ & 2.4453683 & 4.1011560 & 1.0282057 \\
\hline 26 & 0 & 2.8917752 & 5.2245919 & 1.3103421 \\
\hline 27 & $\mathrm{~N}$ & 2.9618148 & 3.3423362 & 0.0478280 \\
\hline 28 & $\mathrm{H}$ & 3.7433698 & 3.7059187 & -0.4995271 \\
\hline 29 & $\mathrm{H}$ & 2.5201548 & 2.4508381 & -0.2101287 \\
\hline 30 & $\mathrm{C}$ & 2.1018648 & 4.6212046 & 5.7412348 \\
\hline 31 & 0 & 2.6639089 & 3.5319422 & 5.6000929 \\
\hline 32 & $\mathrm{~N}$ & 0.7778556 & 4.7493843 & 5.8842118 \\
\hline 33 & $\mathrm{H}$ & 0.3670153 & 5.6868069 & 5.9808039 \\
\hline 34 & $\mathrm{C}$ & -0.0839493 & 3.5907676 & 5.7775797 \\
\hline 35 & $\mathrm{H}$ & -0.0363585 & 3.1650840 & 4.7626933 \\
\hline 36 & $\mathrm{H}$ & -1.1162031 & 3.9181857 & 5.9631067 \\
\hline 37 & $\mathrm{C}$ & 0.2575580 & 2.5095679 & 6.8033837 \\
\hline 38 & 0 & 0.4141299 & 2.7602239 & 7.9976761 \\
\hline 39 & $\mathrm{~N}$ & 0.3125206 & 1.2617615 & 6.2952151 \\
\hline 40 & $\mathrm{H}$ & 0.3484534 & 1.1406140 & 5.2680723 \\
\hline
\end{tabular}




\begin{tabular}{|c|c|c|c|c|}
\hline 41 & $\mathrm{C}$ & 0.6005715 & 0.1354495 & 7.1547376 \\
\hline 42 & $\mathrm{H}$ & -0.1186382 & 0.1223941 & 7.9888043 \\
\hline 43 & $\mathrm{C}$ & 0.5177728 & -1.1845323 & 6.3863849 \\
\hline 44 & $\mathrm{H}$ & 1.2027054 & -1.1732037 & 5.5267149 \\
\hline 45 & $\mathrm{H}$ & 0.8467842 & -1.9951318 & 7.0485496 \\
\hline 46 & $\mathrm{~S}$ & -1.1576461 & -1.6074486 & 5.7355274 \\
\hline 47 & $\mathrm{C}$ & 1.9675639 & 0.2551039 & 7.8729817 \\
\hline 48 & 0 & 2.0204415 & 0.2624034 & 9.0983307 \\
\hline 49 & $\mathrm{~N}$ & 3.0489597 & 0.3152560 & 7.0648400 \\
\hline 50 & $\mathrm{H}$ & 2.9091671 & 0.1438826 & 6.0694055 \\
\hline 51 & $\mathrm{C}$ & 4.4216356 & 0.2836954 & 7.5431262 \\
\hline 52 & $\mathrm{H}$ & 4.4762026 & 0.9175272 & 8.4419551 \\
\hline 53 & $\mathrm{C}$ & 4.9084548 & -1.1534679 & 7.8902522 \\
\hline 54 & $\mathrm{H}$ & 6.0109322 & -1.1076621 & 7.8781204 \\
\hline 55 & 0 & 4.4338824 & -2.0644362 & 6.9202197 \\
\hline 56 & $\mathrm{H}$ & 4.7410093 & -1.7909428 & 6.0390681 \\
\hline 57 & $\mathrm{C}$ & 4.4735968 & -1.6589932 & 9.2577780 \\
\hline 58 & $\mathrm{H}$ & 4.8398284 & -2.6911273 & 9.3889862 \\
\hline 59 & $\mathrm{H}$ & 4.9002784 & -1.0308009 & 10.0551282 \\
\hline 60 & $\mathrm{H}$ & 3.3815324 & -1.6279435 & 9.3646835 \\
\hline 61 & $\mathrm{C}$ & 5.3992626 & 0.8677980 & 6.4924518 \\
\hline 62 & 0 & 6.5707247 & 0.5077522 & 6.4630495 \\
\hline 63 & $\mathrm{~N}$ & 4.8863063 & 1.7480523 & 5.5982040 \\
\hline 64 & $\mathrm{H}$ & 3.9970462 & 2.2252181 & 5.7782088 \\
\hline 65 & $\mathrm{C}$ & 5.6385740 & 2.2056566 & 4.4403201 \\
\hline 66 & $\mathrm{H}$ & 6.3813774 & 1.4300144 & 4.1977075 \\
\hline 67 & $\mathrm{C}$ & 4.6804422 & 2.4651079 & 3.2645611 \\
\hline 68 & $\mathrm{H}$ & 3.8150651 & 3.0334563 & 3.6340629 \\
\hline 69 & $\mathrm{H}$ & 5.1511057 & 3.0782355 & 2.4789168 \\
\hline 70 & $\mathrm{C}$ & 4.2016508 & 1.1672065 & 2.5729903 \\
\hline 71 & $\mathrm{H}$ & 3.2828543 & 1.3537715 & 1.9982158 \\
\hline 72 & $\mathrm{H}$ & 4.9853189 & 0.8650539 & 1.8632845 \\
\hline 73 & $\mathrm{C}$ & 3.9969307 & -0.0003925 & 3.5191961 \\
\hline 74 & 0 & 2.8503470 & -0.1117653 & 4.1468622 \\
\hline 75 & 0 & 4.8909433 & -0.8177621 & 3.7304352 \\
\hline 76 & $\mathrm{C}$ & 4.8258031 & -4.5027313 & 1.4955784 \\
\hline 77 & $\mathrm{H}$ & 4.3115178 & -5.0353096 & 0.6908405 \\
\hline 78 & $\mathrm{H}$ & 4.7360964 & -5.1508090 & 2.3764243 \\
\hline 79 & $\mathrm{~N}$ & 4.1693683 & -3.2160899 & 1.7127527 \\
\hline 80 & $\mathrm{H}$ & 4.5333434 & -2.6150765 & 2.4486176 \\
\hline 81 & $\mathrm{C}$ & 3.3520611 & -2.6136404 & 0.8327702 \\
\hline 82 & $\mathrm{~N}$ & 2.5876607 & -3.2781627 & -0.0156620 \\
\hline 83 & $\mathrm{H}$ & 2.2686282 & -2.7914075 & -0.8629195 \\
\hline 84 & $\mathrm{H}$ & 2.4723318 & -4.3227202 & 0.0196298 \\
\hline
\end{tabular}




\begin{tabular}{cccll}
85 & $\mathrm{~N}$ & 3.3202623 & -1.2617082 & 0.8675590 \\
86 & $\mathrm{H}$ & 2.6930938 & -0.6858208 & 0.2825686 \\
87 & $\mathrm{H}$ & 4.2003545 & -0.8123940 & 1.0757272 \\
88 & $\mathrm{C}$ & -0.1032754 & 0.0008364 & 3.0236112 \\
89 & $\mathrm{C}$ & 0.3112435 & -1.1700017 & 2.4158070 \\
90 & $\mathrm{H}$ & 2.0554430 & 0.4032382 & 3.8113904 \\
91 & $\mathrm{H}$ & -1.8227858 & -0.5629162 & 6.2680460 \\
92 & $\mathrm{H}$ & -1.1840135 & 0.2227174 & 3.0290980 \\
93 & $\mathrm{H}$ & 1.3656228 & -1.4431616 & 2.4051824 \\
94 & $\mathrm{H}$ & -0.4152149 & -1.8987422 & 2.0584495 \\
95 & $\mathrm{O}$ & 0.6707156 & 0.8782744 & 3.5686993 \\
96 & $\mathrm{O}$ & -0.7363923 & 1.2263599 & 0.1205071 \\
97 & $\mathrm{O}$ & 1.6973697 & 0.7142867 & -0.2708531 \\
98 & $\mathrm{O}$ & -0.0769829 & -0.7239300 & -1.3060691 \\
99 & $\mathrm{~S}$ & 0.2962148 & 0.1139066 & -0.0713612 \\
100 & $\mathrm{O}$ & 1.8587815 & -2.0846204 & -2.6387566 \\
101 & $\mathrm{H}$ & 1.0721422 & -1.6740132 & -2.2106395 \\
102 & $\mathrm{H}$ & 1.6799574 & -3.0473709 & -2.7501469 \\
103 & $\mathrm{H}$ & -6.5138569 & 1.0553737 & -3.0122934 \\
104 & $\mathrm{H}$ & -1.6778844 & 3.9994428 & -2.0448568 \\
105 & $\mathrm{H}$ & 0.0142743 & 6.0977063 & 0.6730115 \\
106 & $\mathrm{H}$ & 2.6583415 & 5.5584277 & 5.7375471 \\
107 & $\mathrm{H}$ & 6.2151605 & 3.0948823 & 4.6950605 \\
108 & $\mathrm{H}$ & 5.8720507 & -4.3724308 & 1.2190734 \\
\hline & & & & \\
\hline
\end{tabular}


Int2b

\begin{tabular}{|c|c|c|c|c|}
\hline \multirow{2}{*}{ Number } & \multirow{2}{*}{$\begin{array}{c}\text { Ato } \\
\text { m }\end{array}$} & \multicolumn{3}{|c|}{ Coordinates (Angstroms) } \\
\hline & & $\mathbf{X}$ & $\mathbf{Y}$ & $\mathbf{Z}$ \\
\hline 1 & $\mathrm{C}$ & -5.9034894 & 0.8241402 & -2.1585190 \\
\hline 2 & $\mathrm{H}$ & -5.5204699 & 1.7818434 & -1.7861704 \\
\hline 3 & $\mathrm{H}$ & -6.5235857 & 0.3777147 & -1.3636309 \\
\hline 4 & $\mathrm{~N}$ & -4.7860052 & -0.0314255 & -2.5441187 \\
\hline 5 & $\mathrm{H}$ & -4.8089693 & -0.4134979 & -3.4939984 \\
\hline 6 & $\mathrm{C}$ & -3.5975403 & -0.0429969 & -1.9168205 \\
\hline 7 & $\mathrm{~N}$ & -3.4070423 & 0.6457930 & -0.7853075 \\
\hline 8 & $\mathrm{H}$ & -2.4636639 & 0.7327651 & -0.3835987 \\
\hline 9 & $\mathrm{H}$ & -4.1644596 & 1.1402467 & -0.3055404 \\
\hline 10 & $\mathrm{~N}$ & -2.5840623 & -0.7448323 & -2.4349101 \\
\hline 11 & $\mathrm{H}$ & -1.6834292 & -0.8096195 & -1.9373877 \\
\hline 12 & $\mathrm{H}$ & -2.7272795 & -1.3531755 & -3.2513073 \\
\hline 13 & $\mathrm{C}$ & -1.5718428 & 4.6910983 & -1.2015784 \\
\hline 14 & 0 & -2.1379376 & 5.7786896 & -1.2283420 \\
\hline 15 & $\mathrm{~N}$ & -0.7616692 & 4.3238641 & -0.1781798 \\
\hline 16 & $\mathrm{H}$ & -0.4242962 & 3.3609267 & -0.1500421 \\
\hline 17 & $\mathrm{C}$ & -0.5121536 & 5.2006152 & 0.9653975 \\
\hline 18 & $\mathrm{H}$ & -1.4847127 & 5.5867349 & 1.3165303 \\
\hline 19 & $\mathrm{C}$ & 0.1451766 & 4.4182931 & 2.1172116 \\
\hline 20 & $\mathrm{H}$ & 0.4282623 & 5.1155447 & 2.9191547 \\
\hline 21 & $\mathrm{H}$ & -0.6379462 & 3.7724952 & 2.5463180 \\
\hline 22 & $\mathrm{C}$ & 1.3258929 & 3.4876422 & 1.7766543 \\
\hline 23 & $\mathrm{H}$ & 1.7411974 & 3.1256764 & 2.7316801 \\
\hline 24 & $\mathrm{H}$ & 0.9726590 & 2.5892089 & 1.2571163 \\
\hline 25 & $\mathrm{C}$ & 2.4749210 & 4.1249173 & 1.0120260 \\
\hline 26 & 0 & 2.8903234 & 5.2578722 & 1.2952808 \\
\hline 27 & $\mathrm{~N}$ & 3.0213861 & 3.3770548 & 0.0385845 \\
\hline 28 & $\mathrm{H}$ & 3.8200506 & 3.7403138 & -0.4863858 \\
\hline 29 & $\mathrm{H}$ & 2.6094535 & 2.4769725 & -0.2186807 \\
\hline 30 & $\mathrm{C}$ & 2.0758888 & 4.6417891 & 5.7176404 \\
\hline 31 & 0 & 2.6183806 & 3.5522083 & 5.5298737 \\
\hline 32 & $\mathrm{~N}$ & 0.7559709 & 4.7763706 & 5.9066106 \\
\hline 33 & $\mathrm{H}$ & 0.3507175 & 5.7139518 & 6.0240566 \\
\hline 34 & $\mathrm{C}$ & -0.1128821 & 3.6236159 & 5.8169786 \\
\hline 35 & $\mathrm{H}$ & -0.0978157 & 3.2037220 & 4.7987486 \\
\hline 36 & $\mathrm{H}$ & -1.1380524 & 3.9559143 & 6.0298940 \\
\hline 37 & $\mathrm{C}$ & 0.2510560 & 2.5395847 & 6.8329567 \\
\hline 38 & 0 & 0.4647805 & 2.7963651 & 8.0160699 \\
\hline 39 & $\mathrm{~N}$ & 0.2643395 & 1.2856880 & 6.3372777 \\
\hline 40 & $\mathrm{H}$ & 0.2090005 & 1.1509652 & 5.3241200 \\
\hline
\end{tabular}




\begin{tabular}{|c|c|c|c|c|}
\hline 41 & $\mathrm{C}$ & 0.6122317 & 0.1575735 & 7.1743807 \\
\hline 42 & $\mathrm{H}$ & -0.0900058 & 0.0967141 & 8.0210361 \\
\hline 43 & $\mathrm{C}$ & 0.5752808 & -1.1466928 & 6.3758163 \\
\hline 44 & $\mathrm{H}$ & 1.2373285 & -1.0717985 & 5.5006555 \\
\hline 45 & $\mathrm{H}$ & 0.9698610 & -1.9550727 & 7.0035988 \\
\hline 46 & $\mathrm{~S}$ & -1.1039590 & -1.6377891 & 5.7777643 \\
\hline 47 & $\mathrm{C}$ & 1.9898787 & 0.3074465 & 7.8631064 \\
\hline 48 & 0 & 2.0655027 & 0.2222842 & 9.0863547 \\
\hline 49 & $\mathrm{~N}$ & 3.0399714 & 0.4723244 & 7.0340635 \\
\hline 50 & $\mathrm{H}$ & 2.8711397 & 0.3600047 & 6.0255292 \\
\hline 51 & $\mathrm{C}$ & 4.4188439 & 0.3423898 & 7.4787085 \\
\hline 52 & $\mathrm{H}$ & 4.5202255 & 0.8855565 & 8.4300201 \\
\hline 53 & $\mathrm{C}$ & 4.8297329 & -1.1543482 & 7.6874782 \\
\hline 54 & $\mathrm{H}$ & 5.9194550 & -1.1922517 & 7.5185635 \\
\hline 55 & 0 & 4.1456027 & -1.9692087 & 6.7647844 \\
\hline 56 & $\mathrm{H}$ & 4.3586290 & -1.6912159 & 5.8526316 \\
\hline 57 & $\mathrm{C}$ & 4.5586915 & -1.6895888 & 9.0880585 \\
\hline 58 & $\mathrm{H}$ & 4.8505833 & -2.7526538 & 9.1278434 \\
\hline 59 & $\mathrm{H}$ & 5.1552300 & -1.1364512 & 9.8310310 \\
\hline 60 & $\mathrm{H}$ & 3.5001410 & -1.5766223 & 9.3578293 \\
\hline 61 & $\mathrm{C}$ & 5.4162510 & 0.9634045 & 6.4769007 \\
\hline 62 & 0 & 6.5986304 & 0.6361515 & 6.4971380 \\
\hline 63 & $\mathrm{~N}$ & 4.9153642 & 1.8340906 & 5.5694984 \\
\hline 64 & $\mathrm{H}$ & 3.9938929 & 2.2589541 & 5.6987179 \\
\hline 65 & $\mathrm{C}$ & 5.6616259 & 2.2485511 & 4.3961174 \\
\hline 66 & $\mathrm{H}$ & 6.4049886 & 1.4645318 & 4.1829261 \\
\hline 67 & $\mathrm{C}$ & 4.7102949 & 2.4615268 & 3.2066198 \\
\hline 68 & $\mathrm{H}$ & 3.8431339 & 3.0444999 & 3.5506942 \\
\hline 69 & $\mathrm{H}$ & 5.1881572 & 3.0474796 & 2.4037064 \\
\hline 70 & $\mathrm{C}$ & 4.2394144 & 1.1392163 & 2.5717159 \\
\hline 71 & $\mathrm{H}$ & 3.3736620 & 1.3348844 & 1.9221178 \\
\hline 72 & $\mathrm{H}$ & 5.0594382 & 0.7719189 & 1.9342377 \\
\hline 73 & $\mathrm{C}$ & 3.8751918 & 0.0453948 & 3.5844213 \\
\hline 74 & 0 & 2.7539604 & 0.1454280 & 4.1737065 \\
\hline 75 & 0 & 4.7039927 & -0.8757740 & 3.7877343 \\
\hline 76 & $\mathrm{C}$ & 4.8624966 & -4.5385529 & 1.6259272 \\
\hline 77 & $\mathrm{H}$ & 4.3088630 & -5.1757512 & 0.9301312 \\
\hline 78 & $\mathrm{H}$ & 4.8919093 & -5.0962159 & 2.5695020 \\
\hline 79 & $\mathrm{~N}$ & 4.1534609 & -3.2711739 & 1.8058566 \\
\hline 80 & $\mathrm{H}$ & 4.4552195 & -2.6520214 & 2.5591074 \\
\hline 81 & $\mathrm{C}$ & 3.3965341 & -2.6921409 & 0.8595512 \\
\hline 82 & $\mathrm{~N}$ & 2.7471243 & -3.3813732 & -0.0654876 \\
\hline 83 & $\mathrm{H}$ & 2.4225782 & -2.8947473 & -0.9067394 \\
\hline 84 & $\mathrm{H}$ & 2.6310352 & -4.4249719 & -0.0255190 \\
\hline
\end{tabular}




\begin{tabular}{ccccc}
85 & $\mathrm{~N}$ & 3.3079737 & -1.3490737 & 0.8855195 \\
86 & $\mathrm{H}$ & 2.7403859 & -0.7930103 & 0.2395585 \\
87 & $\mathrm{H}$ & 4.0386090 & -0.8471066 & 1.3653011 \\
88 & $\mathrm{C}$ & -0.2479406 & -0.1345305 & 2.9517359 \\
89 & $\mathrm{C}$ & 0.3187901 & -1.2229235 & 2.3753494 \\
90 & $\mathrm{H}$ & 1.4082130 & 0.6597317 & 3.6473933 \\
91 & $\mathrm{H}$ & -1.7898064 & -0.5956072 & 6.2909414 \\
92 & $\mathrm{H}$ & -1.3309970 & 0.0167534 & 2.9457412 \\
93 & $\mathrm{H}$ & 1.3976725 & -1.3734442 & 2.4087326 \\
94 & $\mathrm{H}$ & -0.3068401 & -2.0268122 & 1.9866900 \\
95 & $\mathrm{O}$ & 0.4175338 & 0.8773516 & 3.5172021 \\
96 & $\mathrm{O}$ & -0.7333507 & 1.1554681 & 0.1261607 \\
97 & $\mathrm{O}$ & 1.6971900 & 0.6682438 & -0.3152762 \\
98 & $\mathrm{O}$ & -0.0887461 & -0.8662437 & -1.2102597 \\
99 & $\mathrm{~S}$ & 0.3149781 & 0.0727668 & -0.0744477 \\
100 & $\mathrm{O}$ & 1.8723799 & -2.1508147 & -2.6686174 \\
101 & $\mathrm{H}$ & 1.0867043 & -1.7967221 & -2.2006658 \\
102 & $\mathrm{H}$ & 1.7398798 & -3.1230961 & -2.7718330 \\
103 & $\mathrm{H}$ & -6.5163411 & 1.0596907 & -3.0285741 \\
104 & $\mathrm{H}$ & -1.6787780 & 4.0000147 & -2.0376618 \\
105 & $\mathrm{H}$ & 0.0206230 & 6.1051664 & 0.6721343 \\
106 & $\mathrm{H}$ & 2.6401003 & 5.5743725 & 5.7221811 \\
107 & $\mathrm{H}$ & 6.2342297 & 3.1499225 & 4.6145701 \\
108 & $\mathrm{H}$ & 5.8759852 & -4.3906390 & 1.2530606 \\
\hline & & & &
\end{tabular}


TS3b

\begin{tabular}{|c|c|c|c|c|}
\hline \multirow{2}{*}{ Number } & \multirow{2}{*}{$\begin{array}{c}\text { Ato } \\
\text { m }\end{array}$} & \multicolumn{3}{|c|}{ Coordinates (Angstroms) } \\
\hline & & $\mathbf{X}$ & $\mathbf{Y}$ & $\mathbf{Z}$ \\
\hline 1 & $\mathrm{C}$ & -5.8470558 & 0.8179703 & -2.1830686 \\
\hline 2 & $\mathrm{H}$ & -5.4806070 & 1.7858970 & -1.8198394 \\
\hline 3 & $\mathrm{H}$ & -6.4636154 & 0.3724985 & -1.3845458 \\
\hline 4 & $\mathrm{~N}$ & -4.7106576 & -0.0249042 & -2.5410833 \\
\hline 5 & $\mathrm{H}$ & -4.7005044 & -0.4171414 & -3.4866058 \\
\hline 6 & $\mathrm{C}$ & -3.5555740 & -0.0319000 & -1.8543163 \\
\hline 7 & $\mathrm{~N}$ & -3.4291313 & 0.6622114 & -0.7192712 \\
\hline 8 & $\mathrm{H}$ & -2.4966469 & 0.8193508 & -0.3091746 \\
\hline 9 & $\mathrm{H}$ & -4.2125994 & 1.1580922 & -0.2845198 \\
\hline 10 & $\mathrm{~N}$ & -2.5065075 & -0.7328871 & -2.3100685 \\
\hline 11 & $\mathrm{H}$ & -1.6772405 & -0.8401921 & -1.7089036 \\
\hline 12 & $\mathrm{H}$ & -2.6153291 & -1.3570336 & -3.1190886 \\
\hline 13 & $\mathrm{C}$ & -1.5616685 & 4.7011529 & -1.2063138 \\
\hline 14 & 0 & -2.1267953 & 5.7897835 & -1.2546348 \\
\hline 15 & $\mathrm{~N}$ & -0.7577731 & 4.3493946 & -0.1727806 \\
\hline 16 & $\mathrm{H}$ & -0.4690814 & 3.3703444 & -0.1006654 \\
\hline 17 & $\mathrm{C}$ & -0.5342739 & 5.2457117 & 0.9599594 \\
\hline 18 & $\mathrm{H}$ & -1.5139803 & 5.6387961 & 1.2845598 \\
\hline 19 & $\mathrm{C}$ & 0.1015557 & 4.4973063 & 2.1454143 \\
\hline 20 & $\mathrm{H}$ & 0.3547654 & 5.2174947 & 2.9373388 \\
\hline 21 & $\mathrm{H}$ & -0.6876017 & 3.8536893 & 2.5665560 \\
\hline 22 & $\mathrm{C}$ & 1.3006633 & 3.5703438 & 1.8666609 \\
\hline 23 & $\mathrm{H}$ & 1.7214067 & 3.2686858 & 2.8409209 \\
\hline 24 & $\mathrm{H}$ & 0.9621811 & 2.6415414 & 1.4016103 \\
\hline 25 & $\mathrm{C}$ & 2.4398431 & 4.1735279 & 1.0643419 \\
\hline 26 & 0 & 2.8686702 & 5.3118017 & 1.3015552 \\
\hline 27 & $\mathrm{~N}$ & 2.9595613 & 3.3884712 & 0.1056552 \\
\hline 28 & $\mathrm{H}$ & 3.7439263 & 3.7310113 & -0.4514955 \\
\hline 29 & $\mathrm{H}$ & 2.5336389 & 2.4826414 & -0.1051413 \\
\hline 30 & $\mathrm{C}$ & 2.0771970 & 4.6434706 & 5.6935552 \\
\hline 31 & 0 & 2.6280888 & 3.5590700 & 5.5018451 \\
\hline 32 & $\mathrm{~N}$ & 0.7527146 & 4.7699075 & 5.8569563 \\
\hline 33 & $\mathrm{H}$ & 0.3422738 & 5.7038494 & 5.9842021 \\
\hline 34 & $\mathrm{C}$ & -0.1088948 & 3.6156105 & 5.7225644 \\
\hline 35 & $\mathrm{H}$ & -0.0783635 & 3.2248165 & 4.6932120 \\
\hline 36 & $\mathrm{H}$ & -1.1390514 & 3.9354743 & 5.9309755 \\
\hline 37 & $\mathrm{C}$ & 0.2428548 & 2.5063879 & 6.7111641 \\
\hline 38 & 0 & 0.4170273 & 2.7288286 & 7.9086991 \\
\hline 39 & $\mathrm{~N}$ & 0.2879914 & 1.2621570 & 6.1892478 \\
\hline 40 & $\mathrm{H}$ & 0.2174208 & 1.1136241 & 5.1809507 \\
\hline
\end{tabular}




\begin{tabular}{|c|c|c|c|c|}
\hline 41 & $\mathrm{C}$ & 0.5623726 & 0.1360708 & 7.0558659 \\
\hline 42 & $\mathrm{H}$ & -0.1716097 & 0.1260538 & 7.8769452 \\
\hline 43 & $\mathrm{C}$ & 0.4974990 & -1.1877810 & 6.2970302 \\
\hline 44 & $\mathrm{H}$ & 1.2373258 & -1.1924625 & 5.4829234 \\
\hline 45 & $\mathrm{H}$ & 0.7640665 & -1.9974276 & 6.9875727 \\
\hline 46 & $\mathrm{~S}$ & -1.1425033 & -1.5570872 & 5.5246978 \\
\hline 47 & $\mathrm{C}$ & 1.9172520 & 0.2569125 & 7.7999552 \\
\hline 48 & 0 & 1.9430705 & 0.1611445 & 9.0254329 \\
\hline 49 & $\mathrm{~N}$ & 2.9967859 & 0.4221471 & 7.0103543 \\
\hline 50 & $\mathrm{H}$ & 2.8566132 & 0.3166898 & 5.9957664 \\
\hline 51 & $\mathrm{C}$ & 4.3653326 & 0.3133765 & 7.4931365 \\
\hline 52 & $\mathrm{H}$ & 4.4372199 & 0.8653378 & 8.4430278 \\
\hline 53 & $\mathrm{C}$ & 4.7930353 & -1.1747002 & 7.7241988 \\
\hline 54 & $\mathrm{H}$ & 5.8869259 & -1.1975687 & 7.5824025 \\
\hline 55 & 0 & 4.1451420 & -2.0082449 & 6.7917574 \\
\hline 56 & $\mathrm{H}$ & 4.3732636 & -1.7286909 & 5.8838605 \\
\hline 57 & $\mathrm{C}$ & 4.4950159 & -1.6953931 & 9.1244484 \\
\hline 58 & $\mathrm{H}$ & 4.7851485 & -2.7582105 & 9.1819531 \\
\hline 59 & $\mathrm{H}$ & 5.0796860 & -1.1347491 & 9.8714486 \\
\hline 60 & $\mathrm{H}$ & 3.4318456 & -1.5753596 & 9.3726773 \\
\hline 61 & $\mathrm{C}$ & 5.3811847 & 0.9386085 & 6.5097220 \\
\hline 62 & 0 & 6.5638726 & 0.6149851 & 6.5517757 \\
\hline 63 & $\mathrm{~N}$ & 4.8961401 & 1.8072304 & 5.5921041 \\
\hline 64 & $\mathrm{H}$ & 3.9722084 & 2.2308123 & 5.7033638 \\
\hline 65 & $\mathrm{C}$ & 5.6633701 & 2.2206267 & 4.4315680 \\
\hline 66 & $\mathrm{H}$ & 6.4098141 & 1.4360226 & 4.2320952 \\
\hline 67 & $\mathrm{C}$ & 4.7309083 & 2.4305024 & 3.2272574 \\
\hline 68 & $\mathrm{H}$ & 3.8639667 & 3.0235897 & 3.5538897 \\
\hline 69 & $\mathrm{H}$ & 5.2255063 & 3.0061696 & 2.4270441 \\
\hline 70 & $\mathrm{C}$ & 4.2571321 & 1.1075617 & 2.5941622 \\
\hline 71 & $\mathrm{H}$ & 3.3870671 & 1.3075893 & 1.9511884 \\
\hline 72 & $\mathrm{H}$ & 5.0742473 & 0.7403494 & 1.9526623 \\
\hline 73 & $\mathrm{C}$ & 3.8964298 & 0.0061299 & 3.6008326 \\
\hline 74 & 0 & 2.7622305 & 0.0837262 & 4.1685909 \\
\hline 75 & 0 & 4.7353126 & -0.9035993 & 3.8144661 \\
\hline 76 & $\mathrm{C}$ & 4.8502337 & -4.5320894 & 1.6412044 \\
\hline 77 & $\mathrm{H}$ & 4.2800036 & -5.1603434 & 0.9503388 \\
\hline 78 & $\mathrm{H}$ & 4.8965872 & -5.0972875 & 2.5799438 \\
\hline 79 & $\mathrm{~N}$ & 4.1541761 & -3.2595331 & 1.8414562 \\
\hline 80 & $\mathrm{H}$ & 4.4955754 & -2.6292926 & 2.5689722 \\
\hline 81 & $\mathrm{C}$ & 3.3340401 & -2.6978080 & 0.9376934 \\
\hline 82 & $\mathrm{~N}$ & 2.6301341 & -3.4030427 & 0.0662455 \\
\hline 83 & $\mathrm{H}$ & 2.2502437 & -2.9153385 & -0.7465608 \\
\hline 84 & $\mathrm{H}$ & 2.5452701 & -4.4498069 & 0.0937579 \\
\hline
\end{tabular}




\begin{tabular}{ccccc}
85 & $\mathrm{~N}$ & 3.2284747 & -1.3581054 & 0.9455403 \\
86 & $\mathrm{H}$ & 2.5792126 & -0.8191250 & 0.3651012 \\
87 & $\mathrm{H}$ & 3.9807689 & -0.8290981 & 1.3571042 \\
88 & $\mathrm{C}$ & -0.0381518 & -0.3314822 & 2.4782150 \\
89 & $\mathrm{C}$ & 0.5092170 & -1.6331164 & 2.5338218 \\
90 & $\mathrm{H}$ & 1.4452956 & 0.5133972 & 3.4566043 \\
91 & $\mathrm{H}$ & -1.8778193 & -0.7049610 & 6.2686693 \\
92 & $\mathrm{H}$ & -1.1270412 & -0.2409303 & 2.3773856 \\
93 & $\mathrm{H}$ & 1.5040907 & -1.7898162 & 2.9508872 \\
94 & $\mathrm{H}$ & 0.0053470 & -2.4569734 & 2.0261691 \\
95 & $\mathrm{O}$ & 0.4930203 & 0.7023699 & 3.1619954 \\
96 & $\mathrm{O}$ & -0.8540520 & 1.3666965 & 0.1886151 \\
97 & $\mathrm{O}$ & 1.5641347 & 0.7068319 & -0.0026564 \\
98 & $\mathrm{O}$ & -0.2375786 & -0.9458715 & -0.5605286 \\
99 & $\mathrm{~S}$ & 0.1550618 & 0.2381753 & 0.3096986 \\
100 & $\mathrm{O}$ & 1.6186964 & -2.0109000 & -2.4607698 \\
101 & $\mathrm{H}$ & 0.8986209 & -1.7077366 & -1.8690730 \\
102 & $\mathrm{H}$ & 1.5118185 & -2.9828822 & -2.5783458 \\
103 & $\mathrm{H}$ & -6.4660895 & 1.0413025 & -3.0519686 \\
104 & $\mathrm{H}$ & -1.6646511 & 4.0009713 & -2.0352935 \\
105 & $\mathrm{H}$ & 0.0032935 & 6.1466365 & 0.6642857 \\
106 & $\mathrm{H}$ & 2.6390215 & 5.5772216 & 5.7165654 \\
107 & $\mathrm{H}$ & 6.2337841 & 3.1218768 & 4.6561640 \\
108 & $\mathrm{H}$ & 5.8566688 & -4.3881366 & 1.2482246 \\
\hline
\end{tabular}


Int3b

\begin{tabular}{|c|c|c|c|c|}
\hline \multirow{2}{*}{ Number } & \multirow{2}{*}{$\begin{array}{c}\text { Ato } \\
\text { m }\end{array}$} & \multicolumn{3}{|c|}{ Coordinates (Angstroms) } \\
\hline & & $\mathbf{X}$ & $\mathbf{Y}$ & $\mathbf{Z}$ \\
\hline 1 & $\mathrm{C}$ & -5.8410927 & 0.8224241 & -2.1776805 \\
\hline 2 & $\mathrm{H}$ & -5.4776134 & 1.7928257 & -1.8181587 \\
\hline 3 & $\mathrm{H}$ & -6.4584487 & 0.3790549 & -1.3786844 \\
\hline 4 & $\mathrm{~N}$ & -4.7012891 & -0.0198409 & -2.5261907 \\
\hline 5 & $\mathrm{H}$ & -4.6833479 & -0.4168917 & -3.4692011 \\
\hline 6 & $\mathrm{C}$ & -3.5572362 & -0.0320022 & -1.8227825 \\
\hline 7 & $\mathrm{~N}$ & -3.4432760 & 0.6663647 & -0.6891421 \\
\hline 8 & $\mathrm{H}$ & -2.5193347 & 0.8181590 & -0.2614843 \\
\hline 9 & $\mathrm{H}$ & -4.2307986 & 1.1643050 & -0.2632144 \\
\hline 10 & $\mathrm{~N}$ & -2.5047385 & -0.7413567 & -2.2595479 \\
\hline 11 & $\mathrm{H}$ & -1.6926261 & -0.8549342 & -1.6392674 \\
\hline 12 & $\mathrm{H}$ & -2.6040714 & -1.3657185 & -3.0693248 \\
\hline 13 & $\mathrm{C}$ & -1.5697739 & 4.6991886 & -1.1889515 \\
\hline 14 & 0 & -2.1359297 & 5.7866443 & -1.2444002 \\
\hline 15 & $\mathrm{~N}$ & -0.7656012 & 4.3554586 & -0.1525982 \\
\hline 16 & $\mathrm{H}$ & -0.4767086 & 3.3780903 & -0.0699102 \\
\hline 17 & $\mathrm{C}$ & -0.5430375 & 5.2587917 & 0.9743865 \\
\hline 18 & $\mathrm{H}$ & -1.5231371 & 5.6526157 & 1.2969075 \\
\hline 19 & $\mathrm{C}$ & 0.0947775 & 4.5192947 & 2.1641304 \\
\hline 20 & $\mathrm{H}$ & 0.3411365 & 5.2444739 & 2.9535467 \\
\hline 21 & $\mathrm{H}$ & -0.6913787 & 3.8722409 & 2.5852798 \\
\hline 22 & $\mathrm{C}$ & 1.2994916 & 3.5975091 & 1.8935881 \\
\hline 23 & $\mathrm{H}$ & 1.7220250 & 3.3055227 & 2.8701482 \\
\hline 24 & $\mathrm{H}$ & 0.9665758 & 2.6620157 & 1.4405533 \\
\hline 25 & $\mathrm{C}$ & 2.4357686 & 4.1957827 & 1.0858373 \\
\hline 26 & 0 & 2.8605898 & 5.3380848 & 1.3076942 \\
\hline 27 & $\mathrm{~N}$ & 2.9604565 & 3.4009199 & 0.1364922 \\
\hline 28 & $\mathrm{H}$ & 3.7464730 & 3.7361206 & -0.4227153 \\
\hline 29 & $\mathrm{H}$ & 2.5419110 & 2.4900765 & -0.0574728 \\
\hline 30 & $\mathrm{C}$ & 2.0734900 & 4.6456107 & 5.6901327 \\
\hline 31 & 0 & 2.6273228 & 3.5638648 & 5.4932818 \\
\hline 32 & $\mathrm{~N}$ & 0.7481897 & 4.7684270 & 5.8498996 \\
\hline 33 & $\mathrm{H}$ & 0.3362598 & 5.7009551 & 5.9816426 \\
\hline 34 & $\mathrm{C}$ & -0.1118493 & 3.6137241 & 5.7046577 \\
\hline 35 & $\mathrm{H}$ & -0.0782780 & 3.2293635 & 4.6732605 \\
\hline 36 & $\mathrm{H}$ & -1.1428002 & 3.9314161 & 5.9124954 \\
\hline 37 & $\mathrm{C}$ & 0.2361498 & 2.4974219 & 6.6858952 \\
\hline 38 & 0 & 0.4057177 & 2.7119434 & 7.8860093 \\
\hline 39 & $\mathrm{~N}$ & 0.2826482 & 1.2566174 & 6.1562118 \\
\hline 40 & $\mathrm{H}$ & 0.2125419 & 1.1090346 & 5.1467672 \\
\hline
\end{tabular}




\begin{tabular}{|c|c|c|c|c|}
\hline 41 & $\mathrm{C}$ & 0.5482546 & 0.1284361 & 7.0231442 \\
\hline 42 & $\mathrm{H}$ & -0.1932701 & 0.1166439 & 7.8373185 \\
\hline 43 & $\mathrm{C}$ & 0.4915785 & -1.1944187 & 6.2622053 \\
\hline 44 & $\mathrm{H}$ & 1.2429834 & -1.1977554 & 5.4590176 \\
\hline 45 & $\mathrm{H}$ & 0.7448594 & -2.0054804 & 6.9564011 \\
\hline 46 & $\mathrm{~S}$ & -1.1356555 & -1.5591390 & 5.459735 \\
\hline 47 & $\mathrm{C}$ & 1.8963880 & 0.2491898 & 7.780196 \\
\hline 48 & 0 & 1.9130921 & 0.1429959 & 9.005253 \\
\hline 49 & $\mathrm{~N}$ & 2.9808239 & 0.4270466 & $6.999877^{\prime}$ \\
\hline 50 & $\mathrm{H}$ & 2.8449686 & 0.3306710 & 5.9833084 \\
\hline 51 & $\mathrm{C}$ & 4.3465436 & 0.3157010 & 7.4902360 \\
\hline 52 & $\mathrm{H}$ & 4.4138605 & 0.8627015 & 8.44357 \\
\hline 53 & $\mathrm{C}$ & 4.7717329 & -1.1745800 & 7.716416 \\
\hline 54 & $\mathrm{H}$ & 5.8647856 & -1.2004557 & $7.569242 \mathrm{C}$ \\
\hline 55 & 0 & 4.1170373 & -2.0045664 & 6.7860887 \\
\hline 56 & $\mathrm{H}$ & 4.3415569 & -1.7230954 & 5.877446 \\
\hline 57 & $\mathrm{C}$ & 4.4794264 & -1.6949342 & 9.118044 \\
\hline 58 & $\mathrm{H}$ & 4.7669156 & -2.7586028 & 9.1742970 \\
\hline 59 & $\mathrm{H}$ & 5.0698953 & -1.1360465 & 9.8618643 \\
\hline 60 & $\mathrm{H}$ & 3.4176736 & -1.5714214 & 9.3708874 \\
\hline 61 & $\mathrm{C}$ & 5.3691048 & 0.9443206 & 6.516226 \\
\hline 62 & 0 & 6.5519055 & 0.6212178 & 6.5662145 \\
\hline 63 & $\mathrm{~N}$ & 4.8905967 & 1.8150582 & 5.5974770 \\
\hline 64 & $\mathrm{H}$ & 3.9644180 & 2.2355583 & 5.7010046 \\
\hline 65 & $\mathrm{C}$ & 5.6642295 & 2.2256077 & 4.4406441 \\
\hline 66 & $\mathrm{H}$ & 6.4098610 & 1.4389617 & 4.246118 \\
\hline 67 & $\mathrm{C}$ & 4.7394663 & 2.4369352 & 3.230646 \\
\hline 68 & $\mathrm{H}$ & 3.8714331 & 3.0310041 & 3.5523553 \\
\hline 69 & $\mathrm{H}$ & 5.2396682 & 3.0115200 & 2.4329517 \\
\hline 70 & $\mathrm{C}$ & 4.2667803 & 1.1149759 & 2.5967330 \\
\hline 71 & $\mathrm{H}$ & 3.4049916 & 1.3180963 & 1.944320 \\
\hline 72 & $\mathrm{H}$ & 5.0893704 & 0.7415058 & 1.965941 \\
\hline 73 & $\mathrm{C}$ & 3.8835776 & 0.0216590 & 3.605511 \\
\hline 74 & 0 & 2.7444669 & 0.1146732 & 4.1544028 \\
\hline 75 & 0 & 4.7149794 & -0.8955929 & 3.8310304 \\
\hline 76 & $\mathrm{C}$ & 4.8684399 & -4.5484852 & 1.669947 \\
\hline 77 & $\mathrm{H}$ & 4.2869716 & -5.1858899 & 0.9962865 \\
\hline 78 & $\mathrm{H}$ & 4.9401242 & -5.1044842 & 2.612669 \\
\hline 79 & $\mathrm{~N}$ & 4.1692891 & -3.2792683 & 1.877402 \\
\hline 80 & $\mathrm{H}$ & 4.5118700 & -2.6481337 & 2.6034417 \\
\hline 81 & $\mathrm{C}$ & 3.3447482 & -2.7174920 & 0.9783765 \\
\hline 82 & $\mathrm{~N}$ & 2.6320851 & -3.4227497 & 0.112951 \\
\hline 83 & $\mathrm{H}$ & 2.2469720 & -2.9332014 & -0.695098 \\
\hline 84 & $\mathrm{H}$ & 2.5483361 & -4.4695780 & 0.137059 \\
\hline
\end{tabular}




\begin{tabular}{ccccc}
85 & $\mathrm{~N}$ & 3.2508477 & -1.3797051 & 0.9744163 \\
86 & $\mathrm{H}$ & 2.5623606 & -0.8380015 & 0.4459302 \\
87 & $\mathrm{H}$ & 3.9761942 & -0.8507476 & 1.4302701 \\
88 & $\mathrm{C}$ & -0.0546548 & -0.2849859 & 2.3088424 \\
89 & $\mathrm{C}$ & 0.5258956 & -1.6185681 & 2.4842593 \\
90 & $\mathrm{H}$ & 1.3913527 & 0.5498665 & 3.3700990 \\
91 & $\mathrm{H}$ & -1.8892482 & -0.7338549 & 6.2170722 \\
92 & $\mathrm{H}$ & -1.1572628 & -0.2945543 & 2.3728621 \\
93 & $\mathrm{H}$ & 1.4721586 & -1.7245212 & 3.0143957 \\
94 & $\mathrm{H}$ & 0.0950721 & -2.4756302 & 1.9644489 \\
95 & $\mathrm{O}$ & 0.4460185 & 0.7343634 & 3.0900721 \\
96 & $\mathrm{O}$ & -0.8800515 & 1.3819818 & 0.2865335 \\
97 & $\mathrm{O}$ & 1.5180121 & 0.6736526 & 0.1770198 \\
98 & $\mathrm{O}$ & -0.2975305 & -0.9505281 & -0.3937058 \\
99 & $\mathrm{~S}$ & 0.0985296 & 0.2462554 & 0.4395210 \\
100 & $\mathrm{O}$ & 1.5575711 & -1.9976560 & -2.3850816 \\
101 & $\mathrm{H}$ & 0.8481090 & -1.7223855 & -1.7703607 \\
102 & $\mathrm{H}$ & 1.4726550 & -2.9711307 & -2.5098984 \\
103 & $\mathrm{H}$ & -6.4593125 & 1.0415299 & -3.0482344 \\
104 & $\mathrm{H}$ & -1.6709232 & 3.9948337 & -2.0146150 \\
105 & $\mathrm{H}$ & -0.0060285 & 6.1582883 & 0.6733976 \\
106 & $\mathrm{H}$ & 2.6347293 & 5.5795398 & 5.7193555 \\
107 & $\mathrm{H}$ & 6.2361240 & 3.1258125 & 4.6656666 \\
108 & $\mathrm{H}$ & 5.8654139 & -4.3985125 & 1.2556787 \\
\hline & & & &
\end{tabular}


TS4b

\begin{tabular}{|c|c|c|c|c|}
\hline \multirow{2}{*}{ Number } & \multirow{2}{*}{$\begin{array}{c}\text { Ato } \\
\text { m }\end{array}$} & \multicolumn{3}{|c|}{ Coordinates (Angstroms) } \\
\hline & & $\mathbf{X}$ & $\mathbf{Y}$ & $\mathbf{Z}$ \\
\hline 1 & $\mathrm{C}$ & -5.854719 & 0.826414 & -2.181846 \\
\hline 2 & $\mathrm{H}$ & -5.488951 & 1.795507 & -1.821379 \\
\hline 3 & $\mathrm{H}$ & -6.470506 & 0.381566 & -1.382558 \\
\hline 4 & $\mathrm{~N}$ & -4.717159 & -0.016028 & -2.538286 \\
\hline 5 & $\mathrm{H}$ & -4.706777 & -0.413143 & -3.481991 \\
\hline 6 & $\mathrm{C}$ & -3.566790 & -0.025438 & -1.846650 \\
\hline 7 & $\mathrm{~N}$ & -3.443964 & 0.671131 & -0.712270 \\
\hline 8 & $\mathrm{H}$ & -2.516695 & 0.823845 & -0.293514 \\
\hline 9 & $\mathrm{H}$ & -4.228827 & 1.165461 & -0.276994 \\
\hline 10 & $\mathrm{~N}$ & -2.516295 & -0.730721 & -2.295165 \\
\hline 11 & $\mathrm{H}$ & -1.697619 & -0.844764 & -1.684449 \\
\hline 12 & $\mathrm{H}$ & -2.621088 & -1.355494 & -3.104497 \\
\hline 13 & $\mathrm{C}$ & -1.569350 & 4.701513 & -1.191371 \\
\hline 14 & 0 & -2.135800 & 5.788717 & -1.244136 \\
\hline 15 & $\mathrm{~N}$ & -0.765799 & 4.355888 & -0.154885 \\
\hline 16 & $\mathrm{H}$ & -0.467572 & 3.381550 & -0.079471 \\
\hline 17 & $\mathrm{C}$ & -0.543903 & 5.256595 & 0.974679 \\
\hline 18 & $\mathrm{H}$ & -1.524254 & 5.650296 & 1.295840 \\
\hline 19 & $\mathrm{C}$ & 0.090154 & 4.513511 & 2.164705 \\
\hline 20 & $\mathrm{H}$ & 0.342848 & 5.237429 & 2.953288 \\
\hline 21 & $\mathrm{H}$ & -0.699620 & 3.872314 & 2.588258 \\
\hline 22 & $\mathrm{C}$ & 1.289691 & 3.584502 & 1.893082 \\
\hline 23 & $\mathrm{H}$ & 1.713271 & 3.292543 & 2.869403 \\
\hline 24 & $\mathrm{H}$ & 0.951554 & 2.650055 & 1.439548 \\
\hline 25 & $\mathrm{C}$ & 2.425824 & 4.182379 & 1.082750 \\
\hline 26 & 0 & 2.855199 & 5.321894 & 1.308349 \\
\hline 27 & $\mathrm{~N}$ & 2.943022 & 3.392572 & 0.124985 \\
\hline 28 & $\mathrm{H}$ & 3.727768 & 3.729879 & -0.434581 \\
\hline 29 & $\mathrm{H}$ & 2.526004 & 2.481823 & -0.071118 \\
\hline 30 & $\mathrm{C}$ & 2.077290 & 4.666907 & 5.686970 \\
\hline 31 & 0 & 2.628425 & 3.583057 & 5.493885 \\
\hline 32 & $\mathrm{~N}$ & 0.752915 & 4.793932 & 5.849590 \\
\hline 33 & $\mathrm{H}$ & 0.342531 & 5.727482 & 5.981252 \\
\hline 34 & $\mathrm{C}$ & -0.109077 & 3.638638 & 5.733081 \\
\hline 35 & $\mathrm{H}$ & -0.089897 & 3.239267 & 4.706645 \\
\hline 36 & $\mathrm{H}$ & -1.137339 & 3.958352 & 5.950054 \\
\hline 37 & $\mathrm{C}$ & 0.256633 & 2.541404 & 6.729354 \\
\hline 38 & 0 & 0.435279 & 2.779733 & 7.922892 \\
\hline 39 & $\mathrm{~N}$ & 0.315031 & 1.294888 & 6.218432 \\
\hline 40 & $\mathrm{H}$ & 0.242217 & 1.146094 & 5.213361 \\
\hline
\end{tabular}




\begin{tabular}{|c|c|c|c|c|}
\hline 41 & $\mathrm{C}$ & 0.597853 & 0.173225 & 7.087813 \\
\hline 42 & $\mathrm{H}$ & -0.138983 & 0.154801 & 7.904072 \\
\hline 43 & $\mathrm{C}$ & 0.535930 & -1.147549 & 6.316436 \\
\hline 44 & $\mathrm{H}$ & 1.241128 & -1.135258 & 5.473430 \\
\hline 45 & $\mathrm{H}$ & 0.853162 & -1.961935 & 6.980139 \\
\hline 46 & $\mathrm{~S}$ & -1.143196 & -1.592506 & 5.729699 \\
\hline 47 & $\mathrm{C}$ & 1.955173 & 0.302563 & 7.814442 \\
\hline 48 & 0 & 2.005674 & 0.188322 & 9.037511 \\
\hline 49 & $\mathrm{~N}$ & 3.021332 & 0.482922 & 7.008504 \\
\hline 50 & $\mathrm{H}$ & 2.856984 & 0.388255 & 5.997891 \\
\hline 51 & $\mathrm{C}$ & 4.393989 & 0.338972 & 7.464937 \\
\hline 52 & $\mathrm{H}$ & 4.491074 & 0.868057 & 8.425083 \\
\hline 53 & $\mathrm{C}$ & 4.795081 & -1.164034 & 7.656073 \\
\hline 54 & $\mathrm{H}$ & 5.883073 & -1.209385 & 7.477634 \\
\hline 55 & 0 & 4.096483 & -1.964464 & 6.732782 \\
\hline 56 & $\mathrm{H}$ & 4.306658 & -1.679542 & 5.820876 \\
\hline 57 & $\mathrm{C}$ & 4.531699 & -1.703310 & 9.056799 \\
\hline 58 & $\mathrm{H}$ & 4.804223 & -2.771844 & 9.087654 \\
\hline 59 & $\mathrm{H}$ & 5.148704 & -1.165422 & 9.794584 \\
\hline 60 & $\mathrm{H}$ & 3.478711 & -1.570365 & 9.339517 \\
\hline 61 & $\mathrm{C}$ & 5.401029 & 0.967067 & 6.479173 \\
\hline 62 & 0 & 6.584163 & 0.643474 & 6.510276 \\
\hline 63 & $\mathrm{~N}$ & 4.905475 & 1.836912 & 5.567312 \\
\hline 64 & $\mathrm{H}$ & 3.984032 & 2.262815 & 5.690740 \\
\hline 65 & $\mathrm{C}$ & 5.657586 & 2.246067 & 4.396930 \\
\hline 66 & $\mathrm{H}$ & 6.400975 & 1.460184 & 4.191717 \\
\hline 67 & $\mathrm{C}$ & 4.712503 & 2.452705 & 3.202553 \\
\hline 68 & $\mathrm{H}$ & 3.850997 & 3.050070 & 3.535469 \\
\hline 69 & $\mathrm{H}$ & 5.200787 & 3.022386 & 2.394142 \\
\hline 70 & $\mathrm{C}$ & 4.227968 & 1.128613 & 2.582246 \\
\hline 71 & $\mathrm{H}$ & 3.367551 & 1.331939 & 1.928793 \\
\hline 72 & $\mathrm{H}$ & 5.046967 & 0.744706 & 1.952727 \\
\hline 73 & $\mathrm{C}$ & 3.835404 & 0.044046 & 3.597203 \\
\hline 74 & 0 & 2.690802 & 0.138906 & 4.133905 \\
\hline 75 & 0 & 4.666965 & -0.871183 & 3.835974 \\
\hline 76 & $\mathrm{C}$ & 4.876054 & -4.555187 & 1.686611 \\
\hline 77 & $\mathrm{H}$ & 4.290414 & -5.212803 & 1.036083 \\
\hline 78 & $\mathrm{H}$ & 4.970035 & -5.090092 & 2.639319 \\
\hline 79 & $\mathrm{~N}$ & 4.165638 & -3.290427 & 1.885126 \\
\hline 80 & $\mathrm{H}$ & 4.495613 & -2.657458 & 2.615111 \\
\hline 81 & $\mathrm{C}$ & 3.354086 & -2.731564 & 0.972754 \\
\hline 82 & $\mathrm{~N}$ & 2.667660 & -3.438960 & 0.088064 \\
\hline 83 & $\mathrm{H}$ & 2.287659 & -2.949616 & -0.722681 \\
\hline 84 & $\mathrm{H}$ & 2.583269 & -4.485967 & 0.112565 \\
\hline
\end{tabular}




\begin{tabular}{ccccc}
85 & $\mathrm{~N}$ & 3.248343 & -1.394679 & 0.975069 \\
86 & $\mathrm{H}$ & 2.571643 & -0.855948 & 0.430081 \\
87 & $\mathrm{H}$ & 3.954280 & -0.862902 & 1.457462 \\
88 & $\mathrm{C}$ & -0.093323 & -0.359203 & 2.232588 \\
89 & $\mathrm{C}$ & 0.492031 & -1.691265 & 2.411160 \\
90 & $\mathrm{H}$ & 1.309326 & 0.493892 & 3.345801 \\
91 & $\mathrm{H}$ & -1.129506 & -0.886772 & 4.582630 \\
92 & $\mathrm{H}$ & -1.199399 & -0.379917 & 2.240935 \\
93 & $\mathrm{H}$ & 1.421246 & -1.790619 & 2.972422 \\
94 & $\mathrm{H}$ & 0.091722 & -2.547871 & 1.866776 \\
95 & $\mathrm{O}$ & 0.352983 & 0.639281 & 3.075668 \\
96 & $\mathrm{O}$ & -0.871795 & 1.363848 & 0.258257 \\
97 & $\mathrm{O}$ & 1.524933 & 0.655154 & 0.161950 \\
98 & $\mathrm{O}$ & -0.286750 & -0.955831 & -0.464407 \\
99 & $\mathrm{~S}$ & 0.102870 & 0.224555 & 0.392120 \\
100 & $\mathrm{O}$ & 1.589493 & -2.024706 & -2.412996 \\
101 & $\mathrm{H}$ & 0.869909 & -1.749778 & -1.810457 \\
102 & $\mathrm{H}$ & 1.505711 & -2.998419 & -2.539441 \\
103 & $\mathrm{H}$ & -6.473752 & 1.047098 & -3.051422 \\
104 & $\mathrm{H}$ & -1.671140 & 3.998750 & -2.018311 \\
105 & $\mathrm{H}$ & -0.006300 & 6.156259 & 0.675255 \\
106 & $\mathrm{H}$ & 2.638952 & 5.600692 & 5.712410 \\
107 & $\mathrm{H}$ & 6.229417 & 3.148681 & 4.612254 \\
108 & $\mathrm{H}$ & 5.865322 & -4.402406 & 1.255251 \\
\hline & & & &
\end{tabular}


Int4b

\begin{tabular}{|c|c|c|c|c|}
\hline \multirow{2}{*}{ Number } & \multirow{2}{*}{$\begin{array}{c}\text { Ato } \\
\text { m }\end{array}$} & \multicolumn{3}{|c|}{ Coordinates (Angstroms) } \\
\hline & & $\mathbf{X}$ & $\mathbf{Y}$ & $\mathbf{Z}$ \\
\hline 1 & $\mathrm{C}$ & -5.8553647 & 0.8272620 & -2.1796646 \\
\hline 2 & $\mathrm{H}$ & -5.4892843 & 1.7973773 & -1.8221761 \\
\hline 3 & $\mathrm{H}$ & -6.4715549 & 0.3855046 & -1.3790076 \\
\hline 4 & $\mathrm{~N}$ & -4.7179777 & -0.0171288 & -2.5320298 \\
\hline 5 & $\mathrm{H}$ & -4.7033429 & -0.4133592 & -3.4759644 \\
\hline 6 & $\mathrm{C}$ & -3.5722742 & -0.0297701 & -1.8326191 \\
\hline 7 & $\mathrm{~N}$ & -3.4556747 & 0.6653765 & -0.6971544 \\
\hline 8 & $\mathrm{H}$ & -2.5307677 & 0.8168713 & -0.2731262 \\
\hline 9 & $\mathrm{H}$ & -4.2422006 & 1.1612693 & -0.2664642 \\
\hline 10 & $\mathrm{~N}$ & -2.5198633 & -0.7369941 & -2.2744419 \\
\hline 11 & $\mathrm{H}$ & -1.7075811 & -0.8543009 & -1.6563356 \\
\hline 12 & $\mathrm{H}$ & -2.6201453 & -1.3600329 & -3.0854602 \\
\hline 13 & $\mathrm{C}$ & -1.5707906 & 4.7000935 & -1.1882441 \\
\hline 14 & 0 & -2.1368286 & 5.7874823 & -1.2420016 \\
\hline 15 & $\mathrm{~N}$ & -0.7656957 & 4.3557489 & -0.1526729 \\
\hline 16 & $\mathrm{H}$ & -0.4717382 & 3.3802921 & -0.0738517 \\
\hline 17 & $\mathrm{C}$ & -0.5428662 & 5.2575023 & 0.9757937 \\
\hline 18 & $\mathrm{H}$ & -1.5228556 & 5.6512954 & 1.2979290 \\
\hline 19 & $\mathrm{C}$ & 0.0931158 & 4.5159859 & 2.1654947 \\
\hline 20 & $\mathrm{H}$ & 0.3431865 & 5.2405651 & 2.9543222 \\
\hline 21 & $\mathrm{H}$ & -0.6947559 & 3.8721148 & 2.5885221 \\
\hline 22 & $\mathrm{C}$ & 1.2951407 & 3.5903656 & 1.8942565 \\
\hline 23 & $\mathrm{H}$ & 1.7185425 & 3.2996329 & 2.8708934 \\
\hline 24 & $\mathrm{H}$ & 0.9597486 & 2.6547406 & 1.4422079 \\
\hline 25 & $\mathrm{C}$ & 2.4307139 & 4.1877275 & 1.0842502 \\
\hline 26 & 0 & 2.8595134 & 5.3278117 & 1.3080427 \\
\hline 27 & $\mathrm{~N}$ & 2.9499156 & 3.3957953 & 0.1289771 \\
\hline 28 & $\mathrm{H}$ & 3.7343326 & 3.7321983 & -0.4316124 \\
\hline 29 & $\mathrm{H}$ & 2.5308173 & 2.4862525 & -0.0673277 \\
\hline 30 & $\mathrm{C}$ & 2.0742170 & 4.6528317 & 5.6825687 \\
\hline 31 & 0 & 2.6281727 & 3.5717343 & 5.4815469 \\
\hline 32 & $\mathrm{~N}$ & 0.7496900 & 4.7756311 & 5.8463231 \\
\hline 33 & $\mathrm{H}$ & 0.3384789 & 5.7077612 & 5.9836718 \\
\hline 34 & $\mathrm{C}$ & -0.1105010 & 3.6190630 & 5.7191134 \\
\hline 35 & $\mathrm{H}$ & -0.0859345 & 3.2258398 & 4.6907632 \\
\hline 36 & $\mathrm{H}$ & -1.1401829 & 3.9366574 & 5.9332083 \\
\hline 37 & $\mathrm{C}$ & 0.2526692 & 2.5142780 & 6.7086569 \\
\hline 38 & 0 & 0.4581359 & 2.7505972 & 7.8993776 \\
\hline 39 & $\mathrm{~N}$ & 0.2827553 & 1.2686763 & 6.1958761 \\
\hline 40 & $\mathrm{H}$ & 0.1342062 & 1.1083581 & 5.1986251 \\
\hline
\end{tabular}




\begin{tabular}{|c|c|c|c|c|}
\hline 41 & $\mathrm{C}$ & 0.5547145 & 0.1375920 & 7.0562019 \\
\hline 42 & $\mathrm{H}$ & -0.1845298 & 0.1074215 & 7.8714352 \\
\hline 43 & $\mathrm{C}$ & 0.5199216 & -1.1735611 & 6.2690178 \\
\hline 44 & $\mathrm{H}$ & 1.2227136 & -1.1134742 & 5.4267843 \\
\hline 45 & $\mathrm{H}$ & 0.8589966 & -1.9883842 & 6.9209671 \\
\hline 46 & $\mathrm{~S}$ & -1.1678764 & -1.5513131 & 5.6110616 \\
\hline 47 & $\mathrm{C}$ & 1.9062128 & 0.2617776 & 7.8019898 \\
\hline 48 & 0 & 1.9406977 & 0.1321998 & 9.0239967 \\
\hline 49 & $\mathrm{~N}$ & 2.9813644 & 0.4601559 & 7.0116416 \\
\hline 50 & $\mathrm{H}$ & 2.8311275 & 0.3789606 & 5.9973787 \\
\hline 51 & $\mathrm{C}$ & 4.3509873 & 0.3234876 & 7.4827512 \\
\hline 52 & $\mathrm{H}$ & 4.4381305 & 0.8578882 & 8.4414109 \\
\hline 53 & $\mathrm{C}$ & 4.7575658 & -1.1773925 & 7.6881972 \\
\hline 54 & $\mathrm{H}$ & 5.8432341 & -1.2233689 & 7.4983928 \\
\hline 55 & 0 & 4.0520585 & -1.9917194 & 6.7812428 \\
\hline 56 & $\mathrm{H}$ & 4.2637066 & -1.7201205 & 5.8666336 \\
\hline 57 & $\mathrm{C}$ & 4.5114789 & -1.6968701 & 9.0991668 \\
\hline 58 & $\mathrm{H}$ & 4.7826354 & -2.7654422 & 9.1423689 \\
\hline 59 & $\mathrm{H}$ & 5.1407638 & -1.1502511 & 9.8199906 \\
\hline 60 & $\mathrm{H}$ & 3.4625550 & -1.5558451 & 9.3932499 \\
\hline 61 & $\mathrm{C}$ & 5.3702348 & 0.9489802 & 6.5056268 \\
\hline 62 & 0 & 6.5522699 & 0.6222333 & 6.5511428 \\
\hline 63 & $\mathrm{~N}$ & 4.8895671 & 1.8196952 & 5.5872799 \\
\hline 64 & $\mathrm{H}$ & 3.9680953 & 2.2493538 & 5.6979627 \\
\hline 65 & $\mathrm{C}$ & 5.6599653 & 2.2287976 & 4.4282994 \\
\hline 66 & $\mathrm{H}$ & 6.4043311 & 1.4416100 & 4.2321006 \\
\hline 67 & $\mathrm{C}$ & 4.7315647 & 2.4400152 & 3.2214309 \\
\hline 68 & $\mathrm{H}$ & 3.8662248 & 3.0368813 & 3.5450872 \\
\hline 69 & $\mathrm{H}$ & 5.2308615 & 3.0115286 & 2.4210136 \\
\hline 70 & $\mathrm{C}$ & 4.2536731 & 1.1184709 & 2.5906672 \\
\hline 71 & $\mathrm{H}$ & 3.3957242 & 1.3247360 & 1.9342663 \\
\hline 72 & $\mathrm{H}$ & 5.0761962 & 0.7392739 & 1.9630225 \\
\hline 73 & $\mathrm{C}$ & 3.8586470 & 0.0268390 & 3.5972277 \\
\hline 74 & 0 & 2.7152732 & 0.1220654 & 4.1363849 \\
\hline 75 & 0 & 4.6842595 & -0.8954022 & 3.8259400 \\
\hline 76 & $\mathrm{C}$ & 4.8753775 & -4.5501892 & 1.6825267 \\
\hline 77 & $\mathrm{H}$ & 4.2881030 & -5.2056318 & 1.0313601 \\
\hline 78 & $\mathrm{H}$ & 4.9694524 & -5.0868297 & 2.6343268 \\
\hline 79 & $\mathrm{~N}$ & 4.1680978 & -3.2835979 & 1.8824499 \\
\hline 80 & $\mathrm{H}$ & 4.5025524 & -2.6488045 & 2.6094018 \\
\hline 81 & $\mathrm{C}$ & 3.3466334 & -2.7284664 & 0.9771039 \\
\hline 82 & $\mathrm{~N}$ & 2.6507462 & -3.4380739 & 0.1015276 \\
\hline 83 & $\mathrm{H}$ & 2.2678040 & -2.9499578 & -0.7087206 \\
\hline 84 & $\mathrm{H}$ & 2.5722253 & -4.4857217 & 0.1242837 \\
\hline
\end{tabular}




\begin{tabular}{ccccc}
85 & $\mathrm{~N}$ & 3.2359799 & -1.3913581 & 0.9778062 \\
86 & $\mathrm{H}$ & 2.5560223 & -0.8565445 & 0.4326423 \\
87 & $\mathrm{H}$ & 3.9522277 & -0.8551687 & 1.4398801 \\
88 & $\mathrm{C}$ & -0.0827301 & -0.2995282 & 2.2868269 \\
89 & $\mathrm{C}$ & 0.4937859 & -1.6379379 & 2.4623958 \\
90 & $\mathrm{H}$ & 1.3517306 & 0.5414038 & 3.3648912 \\
91 & $\mathrm{H}$ & -0.7396427 & -2.0160437 & 4.4086052 \\
92 & $\mathrm{H}$ & -1.1871440 & -0.3075890 & 2.3299337 \\
93 & $\mathrm{H}$ & 1.4467913 & -1.7402656 & 2.9825840 \\
94 & $\mathrm{H}$ & 0.0970027 & -2.4811494 & 1.8936809 \\
95 & $\mathrm{O}$ & 0.4018000 & 0.7101180 & 3.0862502 \\
96 & $\mathrm{O}$ & -0.8856395 & 1.3703621 & 0.2700335 \\
97 & $\mathrm{O}$ & 1.5100367 & 0.6574595 & 0.1710907 \\
98 & $\mathrm{O}$ & -0.3059992 & -0.9638638 & -0.4087853 \\
99 & $\mathrm{~S}$ & 0.0892000 & 0.2339251 & 0.4215466 \\
100 & $\mathrm{O}$ & 1.5689405 & -2.0188490 & -2.3880349 \\
101 & $\mathrm{H}$ & 0.8498574 & -1.7496368 & -1.7825323 \\
102 & $\mathrm{H}$ & 1.4872934 & -2.9918671 & -2.5208731 \\
103 & $\mathrm{H}$ & -6.4745458 & 1.0461829 & -3.0495816 \\
104 & $\mathrm{H}$ & -1.6738164 & 3.9963345 & -2.0141836 \\
105 & $\mathrm{H}$ & -0.0054254 & 6.1569190 & 0.6753372 \\
106 & $\mathrm{H}$ & 2.6354624 & 5.5867180 & 5.7130163 \\
107 & $\mathrm{H}$ & 6.2323907 & 3.1292329 & 4.6510380 \\
108 & $\mathrm{H}$ & 5.8646702 & -4.3998023 & 1.2503799 \\
\hline
\end{tabular}


TS5b

\begin{tabular}{|c|c|c|c|c|}
\hline \multirow{2}{*}{ Number } & \multirow{2}{*}{$\begin{array}{c}\text { Ato } \\
\text { m }\end{array}$} & \multicolumn{3}{|c|}{ Coordinates (Angstroms) } \\
\hline & & $\mathbf{X}$ & $\mathbf{Y}$ & $\mathbf{Z}$ \\
\hline 1 & $\mathrm{C}$ & -5.8520963 & 0.8329716 & -2.1820778 \\
\hline 2 & $\mathrm{H}$ & -5.4854905 & 1.8048456 & -1.8297043 \\
\hline 3 & $\mathrm{H}$ & -6.4685486 & 0.3953761 & -1.3792882 \\
\hline 4 & $\mathrm{~N}$ & -4.7141615 & -0.0134629 & -2.5281000 \\
\hline 5 & $\mathrm{H}$ & -4.6958396 & -0.4147776 & -3.4697479 \\
\hline 6 & $\mathrm{C}$ & -3.5748015 & -0.0262131 & -1.8193871 \\
\hline 7 & $\mathrm{~N}$ & -3.4667108 & 0.6711629 & -0.6849720 \\
\hline 8 & $\mathrm{H}$ & -2.5427393 & 0.8317759 & -0.2621239 \\
\hline 9 & $\mathrm{H}$ & -4.2556532 & 1.1682067 & -0.2597956 \\
\hline 10 & $\mathrm{~N}$ & -2.5178698 & -0.7361030 & -2.2492855 \\
\hline 11 & $\mathrm{H}$ & -1.7220171 & -0.8636615 & -1.6140381 \\
\hline 12 & $\mathrm{H}$ & -2.6111248 & -1.3605852 & -3.0599791 \\
\hline 13 & $\mathrm{C}$ & -1.5673374 & 4.7091409 & -1.1984933 \\
\hline 14 & 0 & -2.1376266 & 5.7939485 & -1.2549396 \\
\hline 15 & $\mathrm{~N}$ & -0.7601947 & 4.3698781 & -0.1632209 \\
\hline 16 & $\mathrm{H}$ & -0.4684624 & 3.3942499 & -0.0813367 \\
\hline 17 & $\mathrm{C}$ & -0.5395077 & 5.2707432 & 0.9658771 \\
\hline 18 & $\mathrm{H}$ & -1.5200632 & 5.6584061 & 1.2937265 \\
\hline 19 & $\mathrm{C}$ & 0.1084280 & 4.5293584 & 2.1488020 \\
\hline 20 & $\mathrm{H}$ & 0.3539502 & 5.2506828 & 2.9420583 \\
\hline 21 & $\mathrm{H}$ & -0.6696356 & 3.8729180 & 2.5706392 \\
\hline 22 & $\mathrm{C}$ & 1.3172111 & 3.6184633 & 1.8639158 \\
\hline 23 & $\mathrm{H}$ & 1.7352471 & 3.3077969 & 2.8363244 \\
\hline 24 & $\mathrm{H}$ & 0.9910517 & 2.6905442 & 1.3907525 \\
\hline 25 & $\mathrm{C}$ & 2.4568166 & 4.2330442 & 1.0744830 \\
\hline 26 & 0 & 2.8633960 & 5.3797436 & 1.3031946 \\
\hline 27 & $\mathrm{~N}$ & 3.0102456 & 3.4458486 & 0.1332784 \\
\hline 28 & $\mathrm{H}$ & 3.8079636 & 3.7823736 & -0.4103333 \\
\hline 29 & $\mathrm{H}$ & 2.6041557 & 2.5340040 & -0.0699087 \\
\hline 30 & $\mathrm{C}$ & 2.0738084 & 4.6680262 & 5.6754661 \\
\hline 31 & 0 & 2.6310385 & 3.5890010 & 5.4714771 \\
\hline 32 & $\mathrm{~N}$ & 0.7491875 & 4.7867288 & 5.8370142 \\
\hline 33 & $\mathrm{H}$ & 0.3352946 & 5.7171278 & 5.9779313 \\
\hline 34 & $\mathrm{C}$ & -0.1063104 & 3.6262982 & 5.7118040 \\
\hline 35 & $\mathrm{H}$ & -0.0770364 & 3.2281992 & 4.6854327 \\
\hline 36 & $\mathrm{H}$ & -1.1375028 & 3.9408022 & 5.9229277 \\
\hline 37 & $\mathrm{C}$ & 0.2605338 & 2.5279838 & 6.7073509 \\
\hline 38 & 0 & 0.4649245 & 2.7726901 & 7.8969184 \\
\hline 39 & $\mathrm{~N}$ & 0.2965933 & 1.2815977 & 6.1990214 \\
\hline 40 & $\mathrm{H}$ & 0.1421607 & 1.1222972 & 5.2023992 \\
\hline
\end{tabular}




\begin{tabular}{|c|c|c|c|c|}
\hline 41 & $\mathrm{C}$ & 0.5628109 & 0.1519793 & 7.0616931 \\
\hline 42 & $\mathrm{H}$ & -0.1863611 & 0.1166341 & 7.8665844 \\
\hline 43 & $\mathrm{C}$ & 0.5493916 & -1.1677462 & 6.2824300 \\
\hline 44 & $\mathrm{H}$ & 1.3337005 & -1.1472531 & 5.5122326 \\
\hline 45 & $\mathrm{H}$ & 0.8004631 & -1.9828587 & 6.9724959 \\
\hline 46 & $\mathrm{~S}$ & -1.0589514 & -1.5346849 & 5.4587932 \\
\hline 47 & $\mathrm{C}$ & 1.9083904 & 0.2832604 & 7.8146271 \\
\hline 48 & 0 & 1.9437701 & 0.1414570 & 9.0352246 \\
\hline 49 & $\mathrm{~N}$ & 2.9824872 & 0.4962988 & 7.0251506 \\
\hline 50 & $\mathrm{H}$ & 2.8255466 & 0.4241041 & 6.0122282 \\
\hline 51 & $\mathrm{C}$ & 4.3543467 & 0.3457900 & 7.4835149 \\
\hline 52 & $\mathrm{H}$ & 4.4517708 & 0.8644976 & 8.4494851 \\
\hline 53 & $\mathrm{C}$ & 4.7539884 & -1.1609302 & 7.6605186 \\
\hline 54 & $\mathrm{H}$ & 5.8405681 & -1.2065070 & 7.4753246 \\
\hline 55 & 0 & 4.0495661 & -1.9536098 & 6.7350307 \\
\hline 56 & $\mathrm{H}$ & 4.2661460 & -1.6677541 & 5.8244600 \\
\hline 57 & $\mathrm{C}$ & 4.5007965 & -1.7057389 & 9.0608260 \\
\hline 58 & $\mathrm{H}$ & 4.7705971 & -2.7752077 & 9.0854545 \\
\hline 59 & $\mathrm{H}$ & 5.1268129 & -1.1725794 & 9.7946470 \\
\hline 60 & $\mathrm{H}$ & 3.4507666 & -1.5694928 & 9.3528757 \\
\hline 61 & $\mathrm{C}$ & 5.3709366 & 0.9772065 & 6.5077505 \\
\hline 62 & 0 & 6.5537610 & 0.6531708 & 6.5520277 \\
\hline 63 & $\mathrm{~N}$ & 4.8871914 & 1.8447761 & 5.5871909 \\
\hline 64 & $\mathrm{H}$ & 3.9679493 & 2.2788454 & 5.7004968 \\
\hline 65 & $\mathrm{C}$ & 5.6532758 & 2.2446276 & 4.4222996 \\
\hline 66 & $\mathrm{H}$ & 6.3952977 & 1.4544784 & 4.2288682 \\
\hline 67 & $\mathrm{C}$ & 4.7214100 & 2.4492181 & 3.2170978 \\
\hline 68 & $\mathrm{H}$ & 3.8607145 & 3.0540332 & 3.5381035 \\
\hline 69 & $\mathrm{H}$ & 5.2210398 & 3.0100252 & 2.4093501 \\
\hline 70 & $\mathrm{C}$ & 4.2335980 & 1.1244756 & 2.6007634 \\
\hline 71 & $\mathrm{H}$ & 3.3777008 & 1.3297103 & 1.9415723 \\
\hline 72 & $\mathrm{H}$ & 5.0537887 & 0.7301156 & 1.9797940 \\
\hline 73 & $\mathrm{C}$ & 3.8292249 & 0.0478895 & 3.6191404 \\
\hline 74 & 0 & 2.6793074 & 0.1476852 & 4.1417308 \\
\hline 75 & 0 & 4.6562537 & -0.8683917 & 3.8705435 \\
\hline 76 & $\mathrm{C}$ & 4.8778172 & -4.5667703 & 1.7003889 \\
\hline 77 & $\mathrm{H}$ & 4.2879051 & -5.2272069 & 1.0562966 \\
\hline 78 & $\mathrm{H}$ & 4.9915690 & -5.1032937 & 2.6504018 \\
\hline 79 & $\mathrm{~N}$ & 4.1602969 & -3.3091770 & 1.9150280 \\
\hline 80 & $\mathrm{H}$ & 4.4866593 & -2.6797884 & 2.6496430 \\
\hline 81 & $\mathrm{C}$ & 3.3013385 & -2.7659839 & 1.0393859 \\
\hline 82 & $\mathrm{~N}$ & 2.6239351 & -3.4766454 & 0.1515887 \\
\hline 83 & $\mathrm{H}$ & 2.2122853 & -2.9811272 & -0.6390978 \\
\hline 84 & $\mathrm{H}$ & 2.5668727 & -4.5262380 & 0.1545960 \\
\hline
\end{tabular}




\begin{tabular}{ccccc}
85 & $\mathrm{~N}$ & 3.1321305 & -1.4343022 & 1.0845869 \\
86 & $\mathrm{H}$ & 2.4602219 & -0.9099562 & 0.5208689 \\
87 & $\mathrm{H}$ & 3.8529176 & -0.8843570 & 1.5235420 \\
88 & $\mathrm{C}$ & -0.1979710 & -0.1383920 & 2.3807551 \\
89 & $\mathrm{C}$ & 0.2257073 & -1.5213409 & 2.7067430 \\
90 & $\mathrm{H}$ & 1.3250913 & 0.6397252 & 3.3859862 \\
91 & $\mathrm{H}$ & -0.4814632 & -1.6822441 & 4.1071947 \\
92 & $\mathrm{H}$ & -1.2960566 & -0.0339202 & 2.4175551 \\
93 & $\mathrm{H}$ & 1.2706215 & -1.6571975 & 3.0029208 \\
94 & $\mathrm{H}$ & -0.2314741 & -2.3383590 & 2.1397861 \\
95 & $\mathrm{O}$ & 0.3809588 & 0.8656440 & 3.1240873 \\
96 & $\mathrm{O}$ & -0.9062852 & 1.3932128 & 0.2674943 \\
97 & $\mathrm{O}$ & 1.4735321 & 0.6361812 & 0.2664082 \\
98 & $\mathrm{O}$ & -0.3564556 & -0.9838491 & -0.2704216 \\
99 & $\mathrm{~S}$ & 0.0423792 & 0.2495065 & 0.5016208 \\
100 & $\mathrm{O}$ & 1.5082990 & -2.0267026 & -2.3014132 \\
101 & $\mathrm{H}$ & 0.7886045 & -1.7800887 & -1.6883295 \\
102 & $\mathrm{H}$ & 1.4419226 & -2.9977863 & -2.4546514 \\
103 & $\mathrm{H}$ & -6.4718911 & 1.0482463 & -3.0524675 \\
104 & $\mathrm{H}$ & -1.6669438 & 4.0014620 & -2.0214977 \\
105 & $\mathrm{H}$ & -0.0066055 & 6.1728866 & 0.6655113 \\
106 & $\mathrm{H}$ & 2.6332610 & 5.6028372 & 5.7102250 \\
107 & $\mathrm{H}$ & 6.2276717 & 3.1461044 & 4.6355507 \\
108 & $\mathrm{H}$ & 5.8595556 & -4.4057574 & 1.2550442 \\
\hline
\end{tabular}


Int5b

\begin{tabular}{|c|c|c|c|c|}
\hline \multirow{2}{*}{ Number } & \multirow{2}{*}{$\begin{array}{c}\text { Ato } \\
\text { m }\end{array}$} & \multicolumn{3}{|c|}{ Coordinates (Angstroms) } \\
\hline & & $\mathbf{X}$ & $\mathbf{Y}$ & $\mathbf{Z}$ \\
\hline 1 & $\mathrm{C}$ & -5.8531065 & 0.8261046 & -2.1781796 \\
\hline 2 & $\mathrm{H}$ & -5.4911501 & 1.7956170 & -1.8154080 \\
\hline 3 & $\mathrm{H}$ & -6.4699582 & 0.3790120 & -1.3809552 \\
\hline 4 & $\mathrm{~N}$ & -4.7118579 & -0.0127615 & -2.5309208 \\
\hline 5 & $\mathrm{H}$ & -4.6987219 & -0.4127306 & -3.4732407 \\
\hline 6 & $\mathrm{C}$ & -3.5627488 & -0.0170874 & -1.8366902 \\
\hline 7 & $\mathrm{~N}$ & -3.4428618 & 0.6838760 & -0.7048153 \\
\hline 8 & $\mathrm{H}$ & -2.5165473 & 0.8417862 & -0.2852664 \\
\hline 9 & $\mathrm{H}$ & -4.2304170 & 1.1746032 & -0.2701067 \\
\hline 10 & $\mathrm{~N}$ & -2.5098842 & -0.7221174 & -2.2804786 \\
\hline 11 & $\mathrm{H}$ & -1.6958014 & -0.8347417 & -1.6634697 \\
\hline 12 & $\mathrm{H}$ & -2.6125949 & -1.3497197 & -3.0874461 \\
\hline 13 & $\mathrm{C}$ & -1.5693905 & 4.7026654 & -1.1902506 \\
\hline 14 & 0 & -2.1357976 & 5.7899924 & -1.2464118 \\
\hline 15 & $\mathrm{~N}$ & -0.7669190 & 4.3594983 & -0.1523586 \\
\hline 16 & $\mathrm{H}$ & -0.4751349 & 3.3831633 & -0.0700478 \\
\hline 17 & $\mathrm{C}$ & -0.5492868 & 5.2637499 & 0.9750365 \\
\hline 18 & $\mathrm{H}$ & -1.5308229 & 5.6588859 & 1.2910477 \\
\hline 19 & $\mathrm{C}$ & 0.0805990 & 4.5268936 & 2.1709351 \\
\hline 20 & $\mathrm{H}$ & 0.3296321 & 5.2549094 & 2.9569538 \\
\hline 21 & $\mathrm{H}$ & -0.7102098 & 3.8868646 & 2.5940700 \\
\hline 22 & $\mathrm{C}$ & 1.2813567 & 3.5969952 & 1.9093832 \\
\hline 23 & $\mathrm{H}$ & 1.7040498 & 3.3129481 & 2.8883157 \\
\hline 24 & $\mathrm{H}$ & 0.9437072 & 2.6588403 & 1.4647555 \\
\hline 25 & $\mathrm{C}$ & 2.4179421 & 4.1862188 & 1.0946111 \\
\hline 26 & 0 & 2.8497100 & 5.3269303 & 1.3094201 \\
\hline 27 & $\mathrm{~N}$ & 2.9336367 & 3.3862839 & 0.1441208 \\
\hline 28 & $\mathrm{H}$ & 3.7172145 & 3.7178318 & -0.4200611 \\
\hline 29 & $\mathrm{H}$ & 2.5168721 & 2.4729892 & -0.0395596 \\
\hline 30 & $\mathrm{C}$ & 2.0738760 & 4.6670663 & 5.6832037 \\
\hline 31 & 0 & 2.6284304 & 3.5832622 & 5.5008604 \\
\hline 32 & $\mathrm{~N}$ & 0.7474396 & 4.7930593 & 5.8274989 \\
\hline 33 & $\mathrm{H}$ & 0.3354055 & 5.7256583 & 5.9601438 \\
\hline 34 & $\mathrm{C}$ & -0.1109095 & 3.6352507 & 5.7069941 \\
\hline 35 & $\mathrm{H}$ & -0.0827255 & 3.2312316 & 4.6829897 \\
\hline 36 & $\mathrm{H}$ & -1.1418960 & 3.9524322 & 5.9156326 \\
\hline 37 & $\mathrm{C}$ & 0.2525066 & 2.5425127 & 6.7075252 \\
\hline 38 & 0 & 0.4564474 & 2.7898091 & 7.8962529 \\
\hline 39 & $\mathrm{~N}$ & 0.2843722 & 1.2900074 & 6.2101016 \\
\hline 40 & $\mathrm{H}$ & 0.1377456 & 1.1131640 & 5.2149411 \\
\hline
\end{tabular}




\begin{tabular}{|c|c|c|c|c|}
\hline 41 & $\mathrm{C}$ & 0.5537558 & 0.1818808 & 7.0961914 \\
\hline 42 & $\mathrm{H}$ & -0.1700323 & 0.1891154 & 7.9248931 \\
\hline 43 & $\mathrm{C}$ & 0.4734009 & -1.1607421 & 6.3556169 \\
\hline 44 & $\mathrm{H}$ & 1.1575354 & -1.1323430 & 5.4858572 \\
\hline 45 & $\mathrm{H}$ & 0.8326541 & -1.9616179 & 7.0152686 \\
\hline 46 & $\mathrm{~S}$ & -1.1790894 & -1.5308562 & 5.7015249 \\
\hline 47 & $\mathrm{C}$ & 1.9170778 & 0.2956205 & 7.8203977 \\
\hline 48 & 0 & 1.9620794 & 0.1666868 & 9.0422753 \\
\hline 49 & $\mathrm{~N}$ & 2.9822180 & 0.4824400 & 7.0175157 \\
\hline 50 & $\mathrm{H}$ & 2.8185078 & 0.3971491 & 6.0048325 \\
\hline 51 & $\mathrm{C}$ & 4.3541745 & 0.3296356 & 7.4769195 \\
\hline 52 & $\mathrm{H}$ & 4.4517469 & 0.8551613 & 8.4392051 \\
\hline 53 & $\mathrm{C}$ & 4.7432658 & -1.1782587 & 7.6664464 \\
\hline 54 & $\mathrm{H}$ & 5.8255973 & -1.2375327 & 7.4617170 \\
\hline 55 & 0 & 4.0131960 & -1.9754746 & 6.7647892 \\
\hline 56 & $\mathrm{H}$ & 4.2235798 & -1.7070715 & 5.8477656 \\
\hline 57 & $\mathrm{C}$ & 4.5088146 & -1.7028610 & 9.0776729 \\
\hline 58 & $\mathrm{H}$ & 4.7727231 & -2.7735102 & 9.1127924 \\
\hline 59 & $\mathrm{H}$ & 5.1484283 & -1.1639348 & 9.7952070 \\
\hline 60 & $\mathrm{H}$ & 3.4636366 & -1.5569586 & 9.3830037 \\
\hline 61 & $\mathrm{C}$ & 5.3712711 & 0.9530594 & 6.4978997 \\
\hline 62 & 0 & 6.5515662 & 0.6197456 & 6.5365922 \\
\hline 63 & $\mathrm{~N}$ & 4.8880276 & 1.8287149 & 5.5860652 \\
\hline 64 & $\mathrm{H}$ & 3.9698124 & 2.2629659 & 5.7058217 \\
\hline 65 & $\mathrm{C}$ & 5.6459744 & 2.2315340 & 4.4168054 \\
\hline 66 & $\mathrm{H}$ & 6.3878898 & 1.4429849 & 4.2163636 \\
\hline 67 & $\mathrm{C}$ & 4.7014048 & 2.4351362 & 3.2215421 \\
\hline 68 & $\mathrm{H}$ & 3.8447481 & 3.0410543 & 3.5511079 \\
\hline 69 & $\mathrm{H}$ & 5.1925471 & 2.9959000 & 2.4087432 \\
\hline 70 & $\mathrm{C}$ & 4.2062411 & 1.1105899 & 2.6101307 \\
\hline 71 & $\mathrm{H}$ & 3.3438924 & 1.3160028 & 1.9603941 \\
\hline 72 & $\mathrm{H}$ & 5.0209700 & 0.7229158 & 1.9771505 \\
\hline 73 & $\mathrm{C}$ & 3.8137710 & 0.0246910 & 3.6264214 \\
\hline 74 & 0 & 2.6674024 & 0.1152556 & 4.1595489 \\
\hline 75 & 0 & 4.6488009 & -0.8882146 & 3.8645864 \\
\hline 76 & $\mathrm{C}$ & 4.8824606 & -4.5630794 & 1.6881818 \\
\hline 77 & $\mathrm{H}$ & 4.2950939 & -5.2218894 & 1.0403233 \\
\hline 78 & $\mathrm{H}$ & 4.9832601 & -5.0980356 & 2.6402050 \\
\hline 79 & $\mathrm{~N}$ & 4.1697260 & -3.3008708 & 1.8918719 \\
\hline 80 & $\mathrm{H}$ & 4.4954159 & -2.6720878 & 2.6273226 \\
\hline 81 & $\mathrm{C}$ & 3.3604390 & -2.7376557 & 0.9805403 \\
\hline 82 & $\mathrm{~N}$ & 2.6717480 & -3.4404974 & 0.0936094 \\
\hline 83 & $\mathrm{H}$ & 2.2990061 & -2.9484216 & -0.7191014 \\
\hline 84 & $\mathrm{H}$ & 2.5825828 & -4.4871124 & 0.1188013 \\
\hline
\end{tabular}




\begin{tabular}{ccccc}
85 & $\mathrm{~N}$ & 3.2627761 & -1.4007535 & 0.9829751 \\
86 & $\mathrm{H}$ & 2.5646157 & -0.8547893 & 0.4716024 \\
87 & $\mathrm{H}$ & 3.9580180 & -0.8746787 & 1.4863743 \\
88 & $\mathrm{C}$ & -0.1864606 & -0.2871692 & 2.2535924 \\
89 & $\mathrm{C}$ & 0.3314566 & -1.6955172 & 2.4876866 \\
90 & $\mathrm{H}$ & 1.2703325 & 0.5000333 & 3.3510525 \\
91 & $\mathrm{H}$ & -0.1507236 & -2.1021637 & 3.3901220 \\
92 & $\mathrm{H}$ & -1.2878069 & -0.2542038 & 2.3011016 \\
93 & $\mathrm{H}$ & 1.4156354 & -1.6855770 & 2.6533985 \\
94 & $\mathrm{H}$ & 0.0924596 & -2.3645986 & 1.6502392 \\
95 & $\mathrm{O}$ & 0.3201114 & 0.6840680 & 3.0995712 \\
96 & $\mathrm{O}$ & -0.8750657 & 1.3949904 & 0.2736806 \\
97 & $\mathrm{O}$ & 1.5062245 & 0.6397529 & 0.2678778 \\
98 & $\mathrm{O}$ & -0.3112869 & -0.9391290 & -0.4083814 \\
99 & $\mathrm{~S}$ & 0.0695310 & 0.2386907 & 0.4568741 \\
100 & $\mathrm{O}$ & 1.5657126 & -2.0109789 & -2.3809078 \\
101 & $\mathrm{H}$ & 0.8544411 & -1.7364440 & -1.7682894 \\
102 & $\mathrm{H}$ & 1.4769660 & -2.9834934 & -2.5111691 \\
103 & $\mathrm{H}$ & -6.4705882 & 1.0467073 & -3.0488792 \\
104 & $\mathrm{H}$ & -1.6706421 & 3.9986855 & -2.0162213 \\
105 & $\mathrm{H}$ & -0.0104011 & 6.1624780 & 0.6751080 \\
106 & $\mathrm{H}$ & 2.6355529 & 5.6007517 & 5.7117992 \\
107 & $\mathrm{H}$ & 6.2194155 & 3.1335416 & 4.6303815 \\
108 & $\mathrm{H}$ & 5.8690473 & -4.4066762 & 1.2520031 \\
\hline
\end{tabular}


Prod

\begin{tabular}{|c|c|c|c|c|}
\hline \multirow{2}{*}{ Number } & \multirow{2}{*}{$\begin{array}{c}\text { Ato } \\
\text { m }\end{array}$} & \multicolumn{3}{|c|}{ Coordinates (Angstroms) } \\
\hline & & $\mathbf{X}$ & $\mathbf{Y}$ & $\mathbf{Z}$ \\
\hline 1 & $\mathrm{C}$ & -5.8829625 & 0.8264018 & -2.1713126 \\
\hline 2 & $\mathrm{H}$ & -5.5162678 & 1.7885907 & -1.7936386 \\
\hline 3 & $\mathrm{H}$ & -6.5001816 & 0.3709185 & -1.3789065 \\
\hline 4 & $\mathrm{~N}$ & -4.7510156 & -0.0110502 & -2.5514199 \\
\hline 5 & $\mathrm{H}$ & -4.7655232 & -0.3969607 & -3.4992323 \\
\hline 6 & $\mathrm{C}$ & -3.5554195 & 0.0225287 & -1.9315728 \\
\hline 7 & $\mathrm{~N}$ & -3.3825588 & 0.7236447 & -0.8061128 \\
\hline 8 & $\mathrm{H}$ & -2.4279284 & 0.8963563 & -0.4386604 \\
\hline 9 & $\mathrm{H}$ & -4.1581147 & 1.1920454 & -0.3312810 \\
\hline 10 & $\mathrm{~N}$ & -2.5205604 & -0.6477254 & -2.4491288 \\
\hline 11 & $\mathrm{H}$ & -1.6344019 & -0.7129521 & -1.9096086 \\
\hline 12 & $\mathrm{H}$ & -2.6618333 & -1.2812431 & -3.2450371 \\
\hline 13 & $\mathrm{C}$ & -1.5396813 & 4.7120726 & -1.2470618 \\
\hline 14 & 0 & -2.0742331 & 5.8186689 & -1.2969433 \\
\hline 15 & $\mathrm{~N}$ & -0.7634696 & 4.3274932 & -0.2064124 \\
\hline 16 & $\mathrm{H}$ & -0.5113305 & 3.3307353 & -0.1443872 \\
\hline 17 & $\mathrm{C}$ & -0.5375864 & 5.2119277 & 0.9368290 \\
\hline 18 & $\mathrm{H}$ & -1.5146886 & 5.6132597 & 1.2608833 \\
\hline 19 & $\mathrm{C}$ & 0.0839292 & 4.4460179 & 2.1187151 \\
\hline 20 & $\mathrm{H}$ & 0.3548200 & 5.1563691 & 2.9142047 \\
\hline 21 & $\mathrm{H}$ & -0.7154787 & 3.8146904 & 2.5395657 \\
\hline 22 & $\mathrm{C}$ & 1.2644154 & 3.4994048 & 1.8250076 \\
\hline 23 & $\mathrm{H}$ & 1.6766598 & 3.1777769 & 2.7961240 \\
\hline 24 & $\mathrm{H}$ & 0.9030473 & 2.5901870 & 1.3355910 \\
\hline 25 & $\mathrm{C}$ & 2.4135271 & 4.0968678 & 1.0276622 \\
\hline 26 & 0 & 2.8856117 & 5.2112887 & 1.3042768 \\
\hline 27 & $\mathrm{~N}$ & 2.8883647 & 3.3305948 & 0.0343843 \\
\hline 28 & $\mathrm{H}$ & 3.6565298 & 3.6884489 & -0.5342270 \\
\hline 29 & $\mathrm{H}$ & 2.4260956 & 2.4421242 & -0.2141808 \\
\hline 30 & $\mathrm{C}$ & 2.0934709 & 4.6384157 & 5.6984552 \\
\hline 31 & 0 & 2.6538582 & 3.5490244 & 5.5495598 \\
\hline 32 & $\mathrm{~N}$ & 0.7681228 & 4.7633483 & 5.8311801 \\
\hline 33 & $\mathrm{H}$ & 0.3525741 & 5.6975218 & 5.9426189 \\
\hline 34 & $\mathrm{C}$ & -0.0827689 & 3.5969100 & 5.7433416 \\
\hline 35 & $\mathrm{H}$ & -0.0493027 & 3.1646198 & 4.7302853 \\
\hline 36 & $\mathrm{H}$ & -1.1166378 & 3.9117993 & 5.9422414 \\
\hline 37 & $\mathrm{C}$ & 0.2948847 & 2.5386913 & 6.7770759 \\
\hline 38 & 0 & 0.5148731 & 2.8229784 & 7.9534728 \\
\hline 39 & $\mathrm{~N}$ & 0.3288694 & 1.2692751 & 6.3197356 \\
\hline 40 & $\mathrm{H}$ & 0.1372006 & 1.0687266 & 5.3385818 \\
\hline
\end{tabular}




\begin{tabular}{|c|c|c|c|c|}
\hline 41 & $\mathrm{C}$ & 0.5844440 & 0.1849337 & 7.2383191 \\
\hline 42 & $\mathrm{H}$ & -0.1290482 & 0.2360227 & 8.0747208 \\
\hline 43 & $\mathrm{C}$ & 0.4713149 & -1.1889775 & 6.5617906 \\
\hline 44 & $\mathrm{H}$ & 1.2120728 & -1.2638857 & 5.7471758 \\
\hline 45 & $\mathrm{H}$ & 0.7520917 & -1.9518056 & 7.2989802 \\
\hline 46 & $\mathrm{~S}$ & -1.1615815 & -1.5465791 & 5.8435361 \\
\hline 47 & $\mathrm{C}$ & 1.9606936 & 0.2957247 & 7.9326409 \\
\hline 48 & 0 & 2.0402762 & 0.2028691 & 9.1526898 \\
\hline 49 & $\mathrm{~N}$ & 3.0180252 & 0.4300979 & 7.1024171 \\
\hline 50 & $\mathrm{H}$ & 2.8349069 & 0.3288876 & 6.1061611 \\
\hline 51 & $\mathrm{C}$ & 4.4028234 & 0.2968064 & 7.5235858 \\
\hline 52 & $\mathrm{H}$ & 4.5257698 & 0.8691323 & 8.4549672 \\
\hline 53 & $\mathrm{C}$ & 4.8163613 & -1.1914143 & 7.7702699 \\
\hline 54 & $\mathrm{H}$ & 5.9084986 & -1.2273920 & 7.6185541 \\
\hline 55 & 0 & 4.1529574 & -2.0294993 & 6.8476454 \\
\hline 56 & $\mathrm{H}$ & 4.4157932 & -1.7845090 & 5.9435555 \\
\hline 57 & $\mathrm{C}$ & 4.5234712 & -1.7030135 & 9.1737399 \\
\hline 58 & $\mathrm{H}$ & 4.8238600 & -2.7622037 & 9.2385733 \\
\hline 59 & $\mathrm{H}$ & 5.1011590 & -1.1307966 & 9.9167593 \\
\hline 60 & $\mathrm{H}$ & 3.4599945 & -1.5923792 & 9.4224312 \\
\hline 61 & $\mathrm{C}$ & 5.3805698 & 0.8883044 & 6.4815316 \\
\hline 62 & 0 & 6.5543427 & 0.5375647 & 6.4656092 \\
\hline 63 & $\mathrm{~N}$ & 4.8649303 & 1.7543882 & 5.5719401 \\
\hline 64 & $\mathrm{H}$ & 3.9898838 & 2.2556205 & 5.7578784 \\
\hline 65 & $\mathrm{C}$ & 5.6132544 & 2.1923386 & 4.4035105 \\
\hline 66 & $\mathrm{H}$ & 6.3546509 & 1.4127212 & 4.1695781 \\
\hline 67 & $\mathrm{C}$ & 4.6433710 & 2.4321074 & 3.2338277 \\
\hline 68 & $\mathrm{H}$ & 3.7994250 & 3.0368664 & 3.5939394 \\
\hline 69 & $\mathrm{H}$ & 5.1181688 & 3.0029765 & 2.4198577 \\
\hline 70 & $\mathrm{C}$ & 4.1166884 & 1.1251073 & 2.5906165 \\
\hline 71 & $\mathrm{H}$ & 3.1979695 & 1.3197431 & 2.0171357 \\
\hline 72 & $\mathrm{H}$ & 4.8812279 & 0.7859464 & 1.8772098 \\
\hline 73 & $\mathrm{C}$ & 3.9068893 & -0.0250354 & 3.5573253 \\
\hline 74 & 0 & 2.7456719 & -0.1505954 & 4.1756152 \\
\hline 75 & 0 & 4.8021332 & -0.8270578 & 3.8029198 \\
\hline 76 & $\mathrm{C}$ & 4.7471197 & -4.5181684 & 1.4162103 \\
\hline 77 & $\mathrm{H}$ & 4.2814613 & -4.9724043 & 0.5372983 \\
\hline 78 & $\mathrm{H}$ & 4.5914562 & -5.2371430 & 2.2307915 \\
\hline 79 & $\mathrm{~N}$ & 4.0918609 & -3.2385924 & 1.6802716 \\
\hline 80 & $\mathrm{H}$ & 4.4753445 & -2.6476584 & 2.4132432 \\
\hline 81 & $\mathrm{C}$ & 3.2126788 & -2.6423440 & 0.8569510 \\
\hline 82 & $\mathrm{~N}$ & 2.4330491 & -3.3155603 & 0.0318477 \\
\hline 83 & $\mathrm{H}$ & 2.0662146 & -2.8185945 & -0.7885990 \\
\hline 84 & $\mathrm{H}$ & 2.3633849 & -4.3651963 & 0.0453630 \\
\hline
\end{tabular}




\begin{tabular}{cllll}
85 & $\mathrm{~N}$ & 3.1330495 & -1.2898758 & 0.9282054 \\
86 & $\mathrm{H}$ & 2.5154627 & -0.7228350 & 0.3178057 \\
87 & $\mathrm{H}$ & 4.0210064 & -0.8299344 & 1.0785798 \\
88 & $\mathrm{C}$ & -0.2433054 & -0.1787219 & 2.8170353 \\
89 & $\mathrm{C}$ & 0.0940016 & -1.6242926 & 2.7040124 \\
90 & $\mathrm{H}$ & 1.9435233 & 0.3020059 & 3.7813024 \\
91 & $\mathrm{H}$ & -0.3670595 & -2.1162168 & 3.5929796 \\
92 & $\mathrm{H}$ & -1.2605367 & 0.1264844 & 2.5054507 \\
93 & $\mathrm{H}$ & 1.1784388 & -1.7890767 & 2.7387561 \\
94 & $\mathrm{H}$ & -0.3400641 & -2.0878251 & 1.8086241 \\
95 & $\mathrm{O}$ & 0.4692848 & 0.6619150 & 3.3937141 \\
96 & $\mathrm{O}$ & -0.7976836 & 1.3973096 & 0.0293239 \\
97 & $\mathrm{O}$ & 1.6234515 & 0.7444825 & -0.2594555 \\
98 & $\mathrm{O}$ & -0.1961108 & -0.8223980 & -0.9881182 \\
99 & $\mathrm{~S}$ & 0.2058990 & 0.2214593 & 0.0839014 \\
100 & $\mathrm{O}$ & 1.7025033 & -2.0104878 & -2.5486657 \\
101 & $\mathrm{H}$ & 0.9418706 & -1.6141690 & -2.0576644 \\
102 & $\mathrm{H}$ & 1.5234774 & -2.9678919 & -2.6799263 \\
103 & $\mathrm{H}$ & -6.4980117 & 1.0591017 & -3.0405837 \\
104 & $\mathrm{H}$ & -1.6516351 & 4.0199956 & -2.0816653 \\
105 & $\mathrm{H}$ & 0.0117259 & 6.1111897 & 0.6581699 \\
106 & $\mathrm{H}$ & 2.6504703 & 5.5752856 & 5.7081267 \\
107 & $\mathrm{H}$ & 6.1876685 & 3.0873831 & 4.6422800 \\
108 & $\mathrm{H}$ & 5.8090287 & -4.3863932 & 1.2087211 \\
\hline & & & & \\
\hline & & & \\
96 & & & \\
96 & & & \\
96 & & &
\end{tabular}


TS6b

\begin{tabular}{|c|c|c|c|c|}
\hline \multirow{2}{*}{ Number } & \multirow{2}{*}{$\begin{array}{c}\text { Ato } \\
\text { m }\end{array}$} & \multicolumn{3}{|c|}{ Coordinates (Angstroms) } \\
\hline & & $\mathbf{X}$ & $\mathbf{Y}$ & $\mathbf{Z}$ \\
\hline 1 & $\mathrm{C}$ & -5.9793816 & 0.7926831 & -2.2014559 \\
\hline 2 & $\mathrm{H}$ & -5.6378815 & 1.7435615 & -1.7749027 \\
\hline 3 & $\mathrm{H}$ & -6.6172346 & 0.3001264 & -1.4491144 \\
\hline 4 & $\mathrm{~N}$ & -4.8244310 & -0.0194111 & -2.5711549 \\
\hline 5 & $\mathrm{H}$ & -4.8085762 & -0.4032770 & -3.5217032 \\
\hline 6 & $\mathrm{C}$ & -3.6704310 & 0.0054798 & -1.8904724 \\
\hline 7 & $\mathrm{~N}$ & -3.5741084 & 0.6783616 & -0.7405362 \\
\hline 8 & $\mathrm{H}$ & -2.6500656 & 0.9127761 & -0.3508378 \\
\hline 9 & $\mathrm{H}$ & -4.3740065 & 1.1484531 & -0.3056146 \\
\hline 10 & $\mathrm{~N}$ & -2.5969030 & -0.6540196 & -2.3590756 \\
\hline 11 & $\mathrm{H}$ & -1.7614304 & -0.7426817 & -1.7713255 \\
\hline 12 & $\mathrm{H}$ & -2.6845830 & -1.2760943 & -3.1729150 \\
\hline 13 & $\mathrm{C}$ & -1.5748912 & 4.7203251 & -1.2074819 \\
\hline 14 & 0 & -2.1425439 & 5.8056790 & -1.2603630 \\
\hline 15 & $\mathrm{~N}$ & -0.7660325 & 4.3727669 & -0.1763351 \\
\hline 16 & $\mathrm{H}$ & -0.4914986 & 3.3926092 & -0.1033169 \\
\hline 17 & $\mathrm{C}$ & -0.5226052 & 5.2564767 & 0.9612166 \\
\hline 18 & $\mathrm{H}$ & -1.4948643 & 5.6320325 & 1.3253434 \\
\hline 19 & $\mathrm{C}$ & 0.1611593 & 4.4866078 & 2.1063872 \\
\hline 20 & $\mathrm{H}$ & 0.4191632 & 5.1825219 & 2.9180983 \\
\hline 21 & $\mathrm{H}$ & -0.6030736 & 3.8095544 & 2.5223772 \\
\hline 22 & $\mathrm{C}$ & 1.3678391 & 3.5956122 & 1.7594043 \\
\hline 23 & $\mathrm{H}$ & 1.7610341 & 3.1765481 & 2.7035101 \\
\hline 24 & $\mathrm{H}$ & 1.0492836 & 2.7224427 & 1.1806860 \\
\hline 25 & $\mathrm{C}$ & 2.5358579 & 4.2752428 & 1.0645290 \\
\hline 26 & 0 & 2.8589625 & 5.4410112 & 1.3187537 \\
\hline 27 & $\mathrm{~N}$ & 3.2133342 & 3.5264316 & 0.1719818 \\
\hline 28 & $\mathrm{H}$ & 4.0474472 & 3.8861258 & -0.3056539 \\
\hline 29 & $\mathrm{H}$ & 2.8897964 & 2.5928585 & -0.0516730 \\
\hline 30 & $\mathrm{C}$ & 2.0887222 & 4.6694600 & 5.6985617 \\
\hline 31 & 0 & 2.6617879 & 3.5942695 & 5.5347850 \\
\hline 32 & $\mathrm{~N}$ & 0.7565628 & 4.7791828 & 5.8149475 \\
\hline 33 & $\mathrm{H}$ & 0.3314540 & 5.7056993 & 5.9442249 \\
\hline 34 & $\mathrm{C}$ & -0.0805817 & 3.6075714 & 5.6777382 \\
\hline 35 & $\mathrm{H}$ & -0.0229401 & 3.2023679 & 4.6548362 \\
\hline 36 & $\mathrm{H}$ & -1.1209885 & 3.9082384 & 5.8628765 \\
\hline 37 & $\mathrm{C}$ & 0.2718776 & 2.5202439 & 6.6888526 \\
\hline 38 & 0 & 0.4045165 & 2.7732037 & 7.8873991 \\
\hline 39 & $\mathrm{~N}$ & 0.3630135 & 1.2721758 & 6.1921638 \\
\hline 40 & $\mathrm{H}$ & 0.3725880 & 1.0967155 & 5.1847933 \\
\hline
\end{tabular}




\begin{tabular}{|c|c|c|c|c|}
\hline 41 & $\mathrm{C}$ & 0.6253966 & 0.1733180 & 7.0917878 \\
\hline 42 & $\mathrm{H}$ & -0.1168989 & 0.1845986 & 7.9042347 \\
\hline 43 & $\mathrm{C}$ & 0.5685986 & -1.1696663 & 6.3454646 \\
\hline 44 & $\mathrm{H}$ & 1.1551850 & -1.0767849 & 5.4096434 \\
\hline 45 & $\mathrm{H}$ & 1.0498378 & -1.9526373 & 6.9459940 \\
\hline 46 & $\mathrm{~S}$ & -1.1180830 & -1.6280119 & 5.8680949 \\
\hline 47 & $\mathrm{C}$ & 1.9719425 & 0.2911123 & 7.8451685 \\
\hline 48 & 0 & 1.9945206 & 0.1425498 & 9.0669279 \\
\hline 49 & $\mathrm{~N}$ & 3.0408369 & 0.4979775 & 7.0580236 \\
\hline 50 & $\mathrm{H}$ & 2.8822281 & 0.4231436 & 6.0363218 \\
\hline 51 & $\mathrm{C}$ & 4.4089766 & 0.3227032 & 7.5092134 \\
\hline 52 & $\mathrm{H}$ & 4.5157037 & 0.8223110 & 8.4831271 \\
\hline 53 & $\mathrm{C}$ & 4.7804051 & -1.1956137 & 7.6629645 \\
\hline 54 & $\mathrm{H}$ & 5.8563743 & -1.2692560 & 7.4312910 \\
\hline 55 & 0 & 4.0157570 & -1.9733457 & 6.7727684 \\
\hline 56 & $\mathrm{H}$ & 4.1993048 & -1.6996998 & 5.8502237 \\
\hline 57 & $\mathrm{C}$ & 4.5690269 & -1.7311782 & 9.0743874 \\
\hline 58 & $\mathrm{H}$ & 4.8055980 & -2.8085812 & 9.0931311 \\
\hline 59 & $\mathrm{H}$ & 5.2395088 & -1.2157543 & 9.7811063 \\
\hline 60 & $\mathrm{H}$ & 3.5348832 & -1.5613572 & 9.4054124 \\
\hline 61 & $\mathrm{C}$ & 5.4266773 & 0.9615910 & 6.5407124 \\
\hline 62 & 0 & 6.6071992 & 0.6254056 & 6.5734042 \\
\hline 63 & $\mathrm{~N}$ & 4.9467895 & 1.8556529 & 5.6468434 \\
\hline 64 & $\mathrm{H}$ & 4.0075887 & 2.2449391 & 5.7447844 \\
\hline 65 & $\mathrm{C}$ & 5.6888690 & 2.2500522 & 4.4661387 \\
\hline 66 & $\mathrm{H}$ & 6.4352210 & 1.4647536 & 4.2691008 \\
\hline 67 & $\mathrm{C}$ & 4.7457838 & 2.4409289 & 3.2660913 \\
\hline 68 & $\mathrm{H}$ & 3.8782841 & 3.0330705 & 3.5941493 \\
\hline 69 & $\mathrm{H}$ & 5.2347464 & 3.0088721 & 2.4560263 \\
\hline 70 & $\mathrm{C}$ & 4.2756750 & 1.1096288 & 2.6585574 \\
\hline 71 & $\mathrm{H}$ & 3.4261858 & 1.3103491 & 1.9882329 \\
\hline 72 & $\mathrm{H}$ & 5.0942094 & 0.6997047 & 2.0485581 \\
\hline 73 & $\mathrm{C}$ & 3.8332113 & 0.0595667 & 3.6988801 \\
\hline 74 & 0 & 2.7735149 & 0.2937144 & 4.3189875 \\
\hline 75 & 0 & 4.5756271 & -0.9642400 & 3.8405688 \\
\hline 76 & $\mathrm{C}$ & 4.8835936 & -4.5912495 & 1.7056076 \\
\hline 77 & $\mathrm{H}$ & 4.3037443 & -5.2827062 & 1.0847345 \\
\hline 78 & $\mathrm{H}$ & 5.0230360 & -5.1017010 & 2.6663109 \\
\hline 79 & $\mathrm{~N}$ & 4.1337145 & -3.3524955 & 1.9116724 \\
\hline 80 & $\mathrm{H}$ & 4.4012871 & -2.7356722 & 2.6823547 \\
\hline 81 & $\mathrm{C}$ & 3.2931369 & -2.8170068 & 1.0186412 \\
\hline 82 & $\mathrm{~N}$ & 2.7348417 & -3.5151598 & 0.0389344 \\
\hline 83 & $\mathrm{H}$ & 2.3205418 & -3.0070096 & -0.7407470 \\
\hline 84 & $\mathrm{H}$ & 2.6727304 & -4.5643032 & 0.0355783 \\
\hline
\end{tabular}




\begin{tabular}{cllll}
85 & $\mathrm{~N}$ & 3.0517061 & -1.4986489 & 1.1038774 \\
86 & $\mathrm{H}$ & 2.2416095 & -1.0475122 & 0.6894526 \\
87 & $\mathrm{H}$ & 3.5207410 & -0.9862347 & 1.8348557 \\
88 & $\mathrm{C}$ & -0.7517511 & -0.5712443 & 2.6281619 \\
89 & $\mathrm{C}$ & -0.3971320 & -2.0304707 & 2.4774190 \\
90 & $\mathrm{H}$ & 0.9162569 & 0.4436533 & 2.2155347 \\
91 & $\mathrm{H}$ & -0.6533734 & -2.5465016 & 3.4183488 \\
92 & $\mathrm{H}$ & -1.8330203 & -0.3515660 & 2.7233470 \\
93 & $\mathrm{H}$ & 0.6826346 & -2.1464232 & 2.3098847 \\
94 & $\mathrm{H}$ & -0.9508517 & -2.5234630 & 1.6672355 \\
95 & $\mathrm{O}$ & 0.0813723 & 0.2418874 & 3.1910808 \\
96 & $\mathrm{O}$ & -1.0522683 & 1.4004244 & 0.1880638 \\
97 & $\mathrm{O}$ & 1.1290987 & 0.5109664 & 1.0583303 \\
98 & $\mathrm{O}$ & -0.2312887 & -0.8542522 & -0.5713283 \\
99 & $\mathrm{~S}$ & -0.3512694 & 0.1215346 & 0.5762837 \\
100 & $\mathrm{O}$ & 1.6112212 & -2.0770385 & -2.4745931 \\
101 & $\mathrm{H}$ & 0.8881324 & -1.7949225 & -1.8840500 \\
102 & $\mathrm{H}$ & 1.5324206 & -3.0534136 & -2.5862993 \\
103 & $\mathrm{H}$ & -6.5665061 & 1.0538180 & -3.0818879 \\
104 & $\mathrm{H}$ & -1.6709394 & 4.0121961 & -2.0305221 \\
105 & $\mathrm{H}$ & 0.0003537 & 6.1649714 & 0.6625222 \\
106 & $\mathrm{H}$ & 2.6389654 & 5.6098148 & 5.7306916 \\
107 & $\mathrm{H}$ & 6.2590360 & 3.1577571 & 4.6637455 \\
108 & $\mathrm{H}$ & 5.8579436 & -4.4157407 & 1.2496406 \\
\hline & & & & \\
\hline
\end{tabular}


Prod'

\begin{tabular}{|c|c|c|c|c|}
\hline \multirow{2}{*}{ Number } & \multirow{2}{*}{$\begin{array}{c}\text { Ato } \\
\text { m }\end{array}$} & \multicolumn{3}{|c|}{ Coordinates (Angstroms) } \\
\hline & & $\mathbf{X}$ & $\mathbf{Y}$ & $\mathbf{Z}$ \\
\hline 1 & $\mathrm{C}$ & -6.0740144 & 0.7309307 & -2.1745275 \\
\hline 2 & $\mathrm{H}$ & -5.7586857 & 1.6480713 & -1.6632000 \\
\hline 3 & $\mathrm{H}$ & -6.7324739 & 0.1786416 & -1.4844678 \\
\hline 4 & $\mathrm{~N}$ & -4.9056083 & -0.0443479 & -2.5833901 \\
\hline 5 & $\mathrm{H}$ & -4.9167644 & -0.3957337 & -3.5470261 \\
\hline 6 & $\mathrm{C}$ & -3.6980403 & 0.0606529 & -2.0062885 \\
\hline 7 & $\mathrm{~N}$ & -3.5401067 & 0.7421853 & -0.8708825 \\
\hline 8 & $\mathrm{H}$ & -2.5998909 & 0.9098342 & -0.4791277 \\
\hline 9 & $\mathrm{H}$ & -4.3258553 & 1.1723521 & -0.3756127 \\
\hline 10 & $\mathrm{~N}$ & -2.6468207 & -0.5402815 & -2.5849016 \\
\hline 11 & $\mathrm{H}$ & -1.6920659 & -0.4299939 & -2.2284179 \\
\hline 12 & $\mathrm{H}$ & -2.7800188 & -1.1802761 & -3.3782303 \\
\hline 13 & $\mathrm{C}$ & -1.5931994 & 4.7370441 & -1.2333176 \\
\hline 14 & 0 & -2.1472973 & 5.8304778 & -1.2764906 \\
\hline 15 & $\mathrm{~N}$ & -0.8031300 & 4.3659114 & -0.1966172 \\
\hline 16 & $\mathrm{H}$ & -0.5108298 & 3.3881687 & -0.1520025 \\
\hline 17 & $\mathrm{C}$ & -0.5606012 & 5.2463878 & 0.9459348 \\
\hline 18 & $\mathrm{H}$ & -1.5331847 & 5.6367048 & 1.2927848 \\
\hline 19 & $\mathrm{C}$ & 0.0957421 & 4.4692753 & 2.1011432 \\
\hline 20 & $\mathrm{H}$ & 0.3871140 & 5.1642417 & 2.9021859 \\
\hline 21 & $\mathrm{H}$ & -0.6861341 & 3.8235355 & 2.5324542 \\
\hline 22 & $\mathrm{C}$ & 1.2642049 & 3.5355769 & 1.7436022 \\
\hline 23 & $\mathrm{H}$ & 1.6540253 & 3.1014483 & 2.6805026 \\
\hline 24 & $\mathrm{H}$ & 0.8898693 & 2.6841583 & 1.1671926 \\
\hline 25 & $\mathrm{C}$ & 2.4428111 & 4.1727444 & 1.0231119 \\
\hline 26 & 0 & 2.8432910 & 5.3090970 & 1.3045125 \\
\hline 27 & $\mathrm{~N}$ & 3.0366618 & 3.4138501 & 0.0823587 \\
\hline 28 & $\mathrm{H}$ & 3.8582121 & 3.7628617 & -0.4182235 \\
\hline 29 & $\mathrm{H}$ & 2.6415120 & 2.5107439 & -0.1702776 \\
\hline 30 & $\mathrm{C}$ & 2.0964086 & 4.6646159 & 5.7326439 \\
\hline 31 & 0 & 2.6698364 & 3.5807861 & 5.6299707 \\
\hline 32 & $\mathrm{~N}$ & 0.7629383 & 4.7763004 & 5.8170144 \\
\hline 33 & $\mathrm{H}$ & 0.3319079 & 5.7044634 & 5.9125377 \\
\hline 34 & $\mathrm{C}$ & -0.0625453 & 3.5948693 & 5.6922958 \\
\hline 35 & $\mathrm{H}$ & 0.0150560 & 3.1660523 & 4.6799211 \\
\hline 36 & $\mathrm{H}$ & -1.1091087 & 3.8875687 & 5.8544174 \\
\hline 37 & $\mathrm{C}$ & 0.2801931 & 2.5337104 & 6.7338458 \\
\hline 38 & 0 & 0.3135850 & 2.7915198 & 7.9358232 \\
\hline 39 & $\mathrm{~N}$ & 0.4616887 & 1.2830540 & 6.2498789 \\
\hline 40 & $\mathrm{H}$ & 0.7409711 & 1.1627497 & 5.2757903 \\
\hline
\end{tabular}




\begin{tabular}{|c|c|c|c|c|}
\hline 41 & $\mathrm{C}$ & 0.7280868 & 0.2147444 & 7.1843196 \\
\hline 42 & $\mathrm{H}$ & -0.0103448 & 0.2757932 & 7.9962611 \\
\hline 43 & $\mathrm{C}$ & 0.6634584 & -1.1673257 & 6.5134914 \\
\hline 44 & $\mathrm{H}$ & 1.2848937 & -1.1428637 & 5.5971654 \\
\hline 45 & $\mathrm{H}$ & 1.1198540 & -1.9132746 & 7.1759388 \\
\hline 46 & $\mathrm{~S}$ & -1.0159174 & -1.6384168 & 6.0327756 \\
\hline 47 & $\mathrm{C}$ & 2.0856064 & 0.3432075 & 7.9094054 \\
\hline 48 & 0 & 2.1383909 & 0.2382103 & 9.1327585 \\
\hline 49 & $\mathrm{~N}$ & 3.1335257 & 0.5063347 & 7.0862876 \\
\hline 50 & $\mathrm{H}$ & 2.9446877 & 0.4007310 & 6.0746980 \\
\hline 51 & $\mathrm{C}$ & 4.5108153 & 0.3228401 & 7.4969395 \\
\hline 52 & $\mathrm{H}$ & 4.6450267 & 0.8213218 & 8.4659113 \\
\hline 53 & $\mathrm{C}$ & 4.8724118 & -1.1957582 & 7.6333681 \\
\hline 54 & $\mathrm{H}$ & 5.9524936 & -1.2695141 & 7.4190145 \\
\hline 55 & 0 & 4.1169227 & -1.9534041 & 6.7189394 \\
\hline 56 & $\mathrm{H}$ & 4.3021907 & -1.6638798 & 5.8000691 \\
\hline 57 & $\mathrm{C}$ & 4.6321678 & -1.7560814 & 9.0306115 \\
\hline 58 & $\mathrm{H}$ & 4.8674529 & -2.8336123 & 9.0331989 \\
\hline 59 & $\mathrm{H}$ & 5.2856201 & -1.2533767 & 9.7618321 \\
\hline 60 & $\mathrm{H}$ & 3.5915900 & -1.5952804 & 9.3456461 \\
\hline 61 & $\mathrm{C}$ & 5.4918998 & 0.9604993 & 6.4936621 \\
\hline 62 & 0 & 6.6718006 & 0.6248483 & 6.4788412 \\
\hline 63 & $\mathrm{~N}$ & 4.9712466 & 1.8528828 & 5.6202342 \\
\hline 64 & $\mathrm{H}$ & 4.0461377 & 2.2591860 & 5.7729880 \\
\hline 65 & $\mathrm{C}$ & 5.6622312 & 2.2598756 & 4.4130861 \\
\hline 66 & $\mathrm{H}$ & 6.4006562 & 1.4781243 & 4.1770634 \\
\hline 67 & $\mathrm{C}$ & 4.6608827 & 2.4534186 & 3.2629101 \\
\hline 68 & $\mathrm{H}$ & 3.8101527 & 3.0455207 & 3.6321915 \\
\hline 69 & $\mathrm{H}$ & 5.1095690 & 3.0208228 & 2.4299479 \\
\hline 70 & $\mathrm{C}$ & 4.1580227 & 1.1226721 & 2.6782394 \\
\hline 71 & $\mathrm{H}$ & 3.2933512 & 1.3326902 & 2.0334030 \\
\hline 72 & $\mathrm{H}$ & 4.9555902 & 0.7170115 & 2.0366576 \\
\hline 73 & $\mathrm{C}$ & 3.7596504 & 0.0596344 & 3.7234141 \\
\hline 74 & 0 & 2.6907394 & 0.2376601 & 4.3556949 \\
\hline 75 & 0 & 4.5513283 & -0.9239260 & 3.8647779 \\
\hline 76 & $\mathrm{C}$ & 4.8555313 & -4.6443637 & 1.6911174 \\
\hline 77 & $\mathrm{H}$ & 4.3067820 & -5.3007303 & 1.0087982 \\
\hline 78 & $\mathrm{H}$ & 4.9389504 & -5.2035397 & 2.6306893 \\
\hline 79 & $\mathrm{~N}$ & 4.0967860 & -3.4140150 & 1.9120891 \\
\hline 80 & $\mathrm{H}$ & 4.3681019 & -2.8121546 & 2.6903368 \\
\hline 81 & $\mathrm{C}$ & 3.3290925 & -2.8237811 & 0.9830205 \\
\hline 82 & $\mathrm{~N}$ & 2.7503952 & -3.4898011 & -0.0058694 \\
\hline 83 & $\mathrm{H}$ & 2.4307656 & -2.9760519 & -0.8331907 \\
\hline 84 & $\mathrm{H}$ & 2.6560168 & -4.5365721 & -0.0014983 \\
\hline
\end{tabular}




\begin{tabular}{cllll}
85 & $\mathrm{~N}$ & 3.1774159 & -1.4945173 & 1.0787916 \\
86 & $\mathrm{H}$ & 2.5232031 & -0.9389334 & 0.5265860 \\
87 & $\mathrm{H}$ & 3.7706906 & -1.0061954 & 1.7328834 \\
88 & $\mathrm{C}$ & -0.5222670 & -0.2928272 & 3.0810875 \\
89 & $\mathrm{C}$ & -0.0046091 & -1.6689618 & 2.7928719 \\
90 & $\mathrm{H}$ & 1.3008154 & 0.6844091 & 1.3276003 \\
91 & $\mathrm{H}$ & -0.3477184 & -2.3830145 & 3.5567620 \\
92 & $\mathrm{H}$ & -1.5570200 & -0.2288658 & 3.4876271 \\
93 & $\mathrm{H}$ & 1.0915177 & -1.6637512 & 2.7212592 \\
94 & $\mathrm{H}$ & -0.4264674 & -2.0231309 & 1.8380432 \\
95 & $\mathrm{O}$ & 0.1136380 & 0.7268769 & 2.8806145 \\
96 & $\mathrm{O}$ & -0.9698266 & 1.2444398 & 0.0027783 \\
97 & $\mathrm{O}$ & 1.4364313 & 0.5585450 & 0.3511600 \\
98 & $\mathrm{O}$ & 0.1026003 & -0.3294925 & -1.6066099 \\
99 & $\mathrm{~S}$ & -0.1400106 & -0.0189284 & -0.1441973 \\
100 & $\mathrm{O}$ & 1.9032332 & -2.1804347 & -2.5820551 \\
101 & $\mathrm{H}$ & 1.0985180 & -1.7411128 & -2.2381273 \\
102 & $\mathrm{H}$ & 1.7172790 & -3.1400719 & -2.7083746 \\
103 & $\mathrm{H}$ & -6.6233091 & 1.0477930 & -3.0610605 \\
104 & $\mathrm{H}$ & -1.6929993 & 4.0421271 & -2.0671027 \\
105 & $\mathrm{H}$ & -0.0202890 & 6.1478715 & 0.6570454 \\
106 & $\mathrm{H}$ & 2.6463234 & 5.6057094 & 5.7346096 \\
107 & $\mathrm{H}$ & 6.2347396 & 3.1685247 & 4.5992531 \\
108 & $\mathrm{H}$ & 5.8507151 & -4.4502860 & 1.2911047 \\
\hline & & & & \\
\hline 9 & & &
\end{tabular}


$\mathrm{TS6}_{\mathrm{w} 2}$

\begin{tabular}{ccccc}
\hline \multirow{2}{*}{ Number } & Ato & \multicolumn{3}{c}{ Coordinates(Angstroms) } \\
\cline { 3 - 5 } & $\mathbf{m}$ & $\mathbf{X}$ & \multicolumn{1}{c}{$\mathbf{Y}$} & \multicolumn{1}{c}{$\mathbf{Z}$} \\
\hline 1 & $\mathrm{C}$ & -0.88568200 & 1.67387000 & 1.33762200 \\
2 & $\mathrm{H}$ & -1.55952100 & 1.68436400 & 2.20869900 \\
3 & $\mathrm{H}$ & -0.05918700 & 2.37626300 & 1.51899900 \\
4 & $\mathrm{H}$ & -1.45911700 & 1.98474900 & 0.45220400 \\
5 & $\mathrm{C}$ & -0.39607500 & 0.24590800 & 1.13802500 \\
6 & $\mathrm{H}$ & 0.26219900 & -0.07353000 & 1.97717400 \\
7 & $\mathrm{O}$ & -1.36328700 & -0.63039200 & 0.81209800 \\
8 & $\mathrm{H}$ & -0.83346000 & -1.88171000 & 0.50609300 \\
9 & $\mathrm{~S}$ & 0.96134800 & 0.34208900 & -0.23235600 \\
10 & $\mathrm{O}$ & 0.34721500 & 0.68525700 & -1.54934700 \\
11 & $\mathrm{O}$ & 2.06488300 & 1.23382400 & 0.19946200 \\
12 & $\mathrm{O}$ & 1.54550900 & -1.12879800 & -0.30160400 \\
13 & $\mathrm{O}$ & -0.23924100 & -2.71871900 & 0.17557600 \\
14 & $\mathrm{H}$ & 0.04922800 & -3.14928600 & 0.99627200 \\
15 & $\mathrm{H}$ & 0.75592500 & -1.91645300 & -0.13603900 \\
\hline
\end{tabular}




\section{References:}

1. Xing, M.; Wei, Y.; Zhou, Y.; Zhang, J.; Lin, L.; Hu, Y.; Hua, G.; N. Nanjaraj Urs, A.; Liu, D.; Wang, F.; Guo, C.; Tong, Y.; Li, M.; Liu, Y.; Ang, E. L.; Zhao, H.; Yuchi, Z.; Zhang, Y. Radical-mediated C-S bond cleavage in C2 sulfonate degradation by anaerobic bacteria. Nat. Commun. 2019, 10, 1609.

2. Brooks, B. R.; Bruccoleri, R. E.; Olafson, B. D.; States, D. J.; Swaminathan, S.; Karplus, M. CHARMM: a program for macromolecular energy, minimization, and dynamics calculations. J. Comput. Chem. 1983, 4, 187-217.

3. Brooks, B. R.; Brooks lii, C. L.; Mackerell Jr, A. D.; Nilsson, L.; Petrella, R. J.; Roux, B.; Won, Y.; Archontis, G.; Bartels, C.; Boresch, S.; Caflisch, A.; Caves, L.; Cui, Q.; Dinner, A. R.; Feig, M.; Fischer, S.; Gao, J.; Hodoscek, M.; Im, W.; Kuczera, K.; Lazaridis, T.; Ma, J.; Ovchinnikov, V.; Paci, E.; Pastor, R. W.; Post, C. B.; Pu, J. Z.; Schaefer, M.; Tidor, B.; Venable, R. M.; Woodcock, H. L.; Wu, X.; Yang, W.; York, D. M.; Karplus, M. CHARMM: the biomolecular simulation program. J. Comput. Chem. 2009, 30, 1545-1614.

4. Wetmore, S. D.; Smith, D. M.; Golding, B. T.; Radom, L. Interconversion of (S)-glutamate and (2S,3S)-3-methylaspartate: a distinctive $\mathrm{B}_{12}$-dependent carbon-skeleton rearrangement. J. Am. Chem. Soc. 2001, 123, 7963-7972.

5. Wetmore, S. D.; Smith, D. M.; Bennett, J. T.; Radom, L. Understanding the mechanism of action of B12-dependent ethanolamine ammonia-lyase synergistic interactions at play. J. Am. Chem. Soc. 2002, 124, 14054-14065.

6. Smith, D. M.; Golding, B. T.; Radom, L. On the mechanism of action of Vitamin $B_{12}$ : theoretical studies of the 2-methyleneglutarate mutase catalyzed rearrangement. J. Am. Chem. Soc. 2002, 124, 14054-14065. 